EXACT RECONSTRUCTION OF OCEAN BOTTOM VELOCITY PROFILES FROM MONOCHROMATIC SCATTERING DATA

\author{
by \\ ANDRE A. MERAB \\ S.M., Massachusetts Institute of Technology, 1979 \\ SUBMITTED IN PARTIAL FULFILLMENT OF THE REQUIREMENTS \\ FOR THE DEGREE OF DOCTOR OF SCIENCE \\ at the \\ MASSACHUSETTS INSTITUTE OF TECHNOLOGY \\ and the \\ WOODS HOLE OCEANOGRAPHIC INSTITUTION
}

\author{
January 1987 \\ ๑ Andre A. Merab, 1987
}

The author hereby grants to MIT and WHOI permission to reproduce and distribute copies of this thesis document in whole or in part.

Siqnature of Author

Department of Electrical Enqineering, Massachusetts Institute of Technology and the Joint Program in Oceanography/Ocean Enqineering, Massachusetts Institute of Technology/Woods Hole Oceanographic Institution.

Certified by

George N. Frisk, Thesis Supervisor

Accepted by

Georgd V. Frisk, Chairman, MIT/WHOI Joint Committee for Oceanographic Engineering 


\title{
Exact Reconstruction of Ocean Bottom Velocity Profiles from Monochromatic Scattering Data
}

\author{
Andre A. Merab \\ Submitted in partial fulfillment of the requirements \\ for the Degree of Doctor of Science \\ at the \\ MASSACHUSETTS INSTITUTE OF TECHNOLOGY \\ and the \\ WOODS HOLE OCEANOGRAPHIC INSTITUTION
}

January 1987

\begin{abstract}
This thesis presents the theoretical and computational underpinninqs of a novel approach to the determination of the acoustic parameters of the ocean bottom using a monochromatic source. The problem is shown to be equivalent to that of the reconstruction of the potential in a Schrodinger equation from the knowledge of the plane-wave reflection coefficient as a function of vertical wavenumber, $r\left(k_{2}\right)$ for all real positive $\mathrm{k}_{\mathrm{z}}$. First, the reflection coefflcient is shown to decay asymptotically at least as fast as $\left(1 / \mathrm{k}^{2}\right)$ for large $\mathrm{k}_{\mathrm{z}}$ and is therefore inteqrable. The Gelfand-Levitan inversion procedure is extended to include the case of basement velocity higher than the velocity of sound in water. The neglect of bound states is shown to be justified in both clayey silt and silty clay at the $220 \mathrm{~Hz}$ frequency of operation.

Three methods for the numerical solution of the integral equation are investigated. The first one is an "Improved Born approximation" wherein the solution is given as a series expansion the first term of which is the Born approximation while the second term represents a substantial and yet easy to implement improvement over Born.

The two other methods are based on a discretization of the Gelfand-Levitan inteqral equation, and both avoid a matrix inversion: one by employing a recursive procedure, and the other by coupling the Gelfand-Levitan equation with a partial differential equation. Bounds are obtained on errors in the solution due either to discretization or to data inaccuracy. These methods are tested on synthetic data obtained from known deoacoustic models of the ocean bottom. Results
\end{abstract}


are found to be very accurate particularly at the top of the sediment layer with resolution of less than the wavelength of the acoustic source in the water. Several effects are investiqated, such as sampling, attenuation, and noise. Also examined is the gradual restriction of the reflection coefficient to a finite ranqe of vertical wavenumbers and the consequent progressive deterioration of the reconstruction.

The analysis shows how to reconstruct velocity profiles in the presence of density variation when the experiment is conducted at two frequencies.

Our results provide a good understanding of the issues involved in conducting a monochromatic deep ocean bottom experiment and constitute a promising technique for processind the experimental data when it becomes available. 


\section{ACKNOWLEDGEMENTS}

It is with deep appreciation and great pleasure that I express my thanks to the people who have contributed to the completion of this work. I am most deeply indebted to my thesis advisor, Dr. George Frisk whose uncanny intuition got this project started, and whose advice, encouragement and good humor kept it going.

I am grateful to Professors Arthur Baggeroer, and Alan oppenheim for serving as thesis readers, and to Dr. James Lynch who served as chairman of the thesis defense.

I wish it were possible to thank all my friends and colleagues at MIT and WHOI. I would like to particularly thank Dr. Douglas Mook, who introduced me to the MIT/WHOI Joint Program, to Dr. Subramaniam Rajan, and to Gengshen Liu for many lively and informative discussions, scientific and otherwise. Many thanks to Dr. David Stickler who shared his ideas and insights on inverse problems with us.

A special thank you to Mrs. Karuna Mohindra, and to Mrs. Margaret Pina who have performed near miracles in typing the manuscript through countless revisions.

Fay, Julian, Jeffrey, Julie and Jessica Bussgang have provided me with a home away from home. My debt to them is beyond words. My uncle, Dr. Julian Bussgang, has freely and cheerfully given of his time to help me bring this work to a conclusion. His advice, encouragement, and enthusiasm, I shall always cherish. Thank you!

Finally, I wish to express my love and thanks to my parents, and to my brother Jacques. Their love, constant encouragement, and unfailing support have been essential all along. To them this work is dedicated.

Support for this thesis was provided in part by the education office at WHOI and by the Office of Naval Research. 
TABLE OF CONTENTS

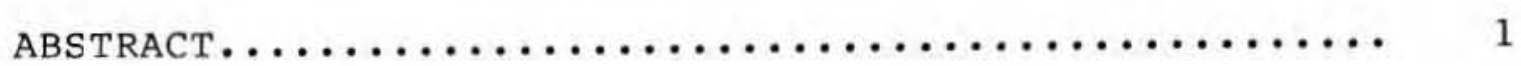

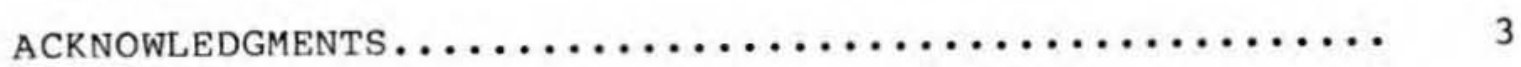

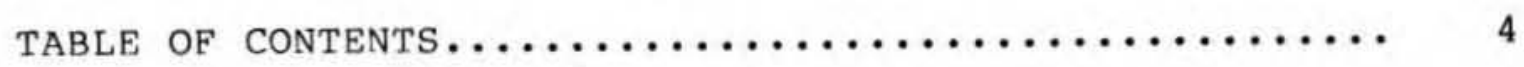

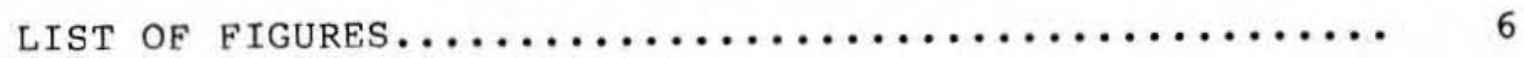

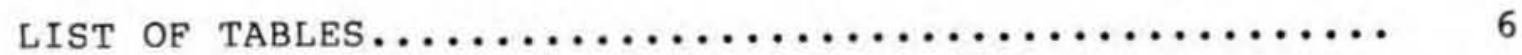

CHAPTER I - BACKGROUND........................... 7

$1.1 \quad$ Background......................... 7

1.2 The Experiment......................... 9

1.3 Experimental Data...................... 11

1.4 The Mode $1 . \ldots \ldots \ldots \ldots \ldots \ldots \ldots \ldots \ldots \ldots \ldots \ldots, 13$

1.5 Overview of the Thesis................ 15

CHAPTER II - PROBLEM FORMULATION AND REVIEW OF PAST WORK $. \ldots \ldots \ldots \ldots \ldots \ldots \ldots, 16$

2.1 Problem Formulation................. 16

2.2 Survey of Inverse Methods.$\ldots \ldots \ldots \ldots \ldots \ldots .24$

2.2 .1 Introduction.................. 24

2.2.2 The Gelfand-Levitan Approach........ 26

2.2.3 Single Frequency, Non-Normal

Incidence...................... 39

2.2.4 The Deift and Trubowitz Method...... 40

2.2 .5 Schur Algorithm.............. 42

2.2.6 Riccati Equation Method............ 46

Chapter III - THE REFlection COefficient............ 51

3.1 Introduction........................ 51

3.2 Definition and Properties of the Reflection Coefficient............................. 51

3.3 Asymptotic Behavior of the Plane Wave Reflection Coefficient................ 55

CHAPTER IV - THE ONE-DIMENSIONAL INVERSE PROBLEM...... 65

4.1 Introduction........................ 66

4.2 Properties of the Solution of the Schrodinger Equation $\left(\mathrm{V}_{1}=0\right) \ldots \ldots \ldots \ldots \ldots \ldots, 67$ 4.2.1 Properties of the Coefficients

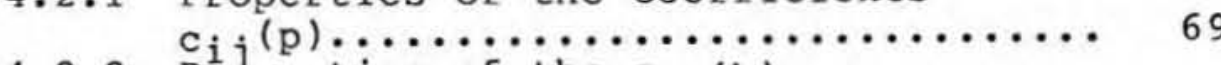

4.2.2 Properties of the $s_{i j}(k)$

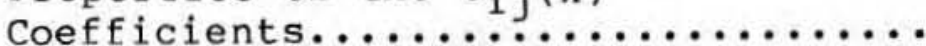


TABLE OF CONTENTS (Concluded)

4.3 Non Zero Final Potential............... 75

4.3.1 Asymptotic Behavior............... 76

4.3.2 Inversion Procedure................ 77

4.4 Bound states....................... 81

CHAPTER V - NUMERICAL SOLUTION OF THE GELFAND-LEVITAN EQUATION.......................... 86

5.1 Introduction....................... 86

5.2 Series Expansion..................... 86

5.2 .1 The Potential................. 88

5.2.2 Connection with Other Formulations... 90

5.2 .3 Algorithm.................. 92

5.3 Uniqueness of the Solution............. 93

5.4 Finite Difference Methods............... 93

5.5 Coupled Equations Method............... 97

5.5 .1 Analysis of Stability ............ 98

5.6 Error Estimates..................... 99

5.6.1 Discretization Errors............... 99

5.6 .2 Data Errors..................... 101

5.6.3 Errors in the Potential........... 103

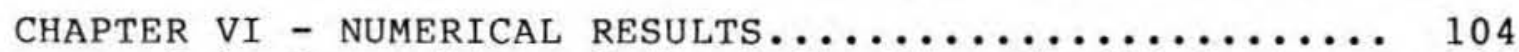

6.1 Generation of the Reflection Coefficient.... 105

6.2 Case of Step Discontinuity in Potential.... 114 6.2.1 Approximate Solution of the GelfandLevitan Equation for a Step

Discontinuity ................. 116

6.3 Fourier Transform of the Reflection

Coefficient....................... 119

6.3 .1 Fast Fourier Transform............ 120

6.3 .2 Limited Angular Aperture........... 121

6.3 .3 Sampling.................... 124

6.4 Frequency Scaling of the Reflection

Coefficient...................... 127

6.5 Profile Reconstruction in the Presence of Density Variations.................. 130

6.6 Acoustic Attenuation................. 132

6.7 Noise............................. 133

CHAPTER VII - CONCLUSION........................ 136

FIgS. $10-31$ NUMERICAL RESUlts.................. 142

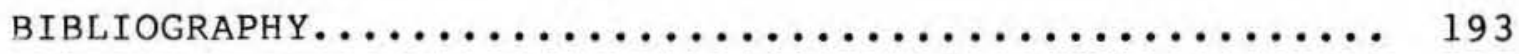




\section{LIST OF FIGURES}

Figure 1 Experimental Configuration........... 10

Figure 2 Schematic Illustration of the

Scattering Problem................ 19

Figure 3 Wavenumber Decomposition............ 20

Figure $4 \quad$ Scattering Potential-Energy Diagram..... 23

Figure 5 Generation of Vertical Wavenumbers...... 30

Figure 6 Mapping from the $\mathrm{k}_{\mathrm{z}}$-Plane into the

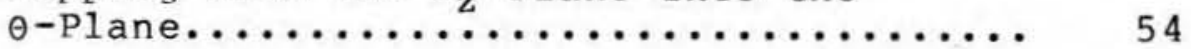

Figure 7 One Layer Case................. 58

Figure $8 \quad$ Stack of Isovelocity Layers........... 62

Figure 9 Adding a Top Layer to the Stack

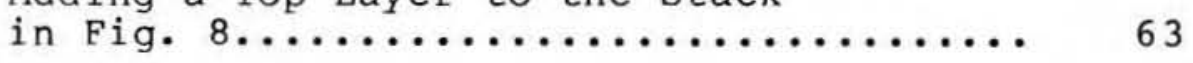

Figure 10-31 Numerical Results................. 142

\section{LIST OF TABLES}

Table I Upper bound on the probing frequency for the condition of zero bound states as a function of velocity ratio, $R$, and velocity

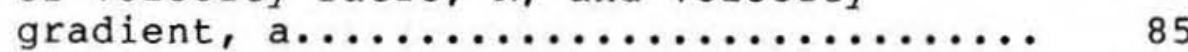


It is a rare person whose pulse is not stirred by the dramatic sight of the restless surface of the sea. The chaotic sea surface is a limitless source of inspiration to poet, painter and musician alike. But what lies beneath this churning surface? How can we probe the depths of the sea?

C. Clay \& H. Medwin

\section{Backqround}

The sea floor begins at the water-sediment interface, overlies the sedimentary layer, and beneath it, the oceanic crust. The study of the ocean bottom has been, until recently, the province of the marine qeologist seeking to probe the oceanic crust, and to unravel the secrets of its structure and evolution. The marine geologist has now been joined by the underwater acoustician studying the transmission of low frequency sound through the ocean; It has become clear in light of underwater sound propagation experiments carried out at the various Oceanographic Institutions (1) that long range low frequency sound transmission is affected by the nature of the ocean bottom, and hence, that acoustic wave propaqation models - to characterize sonar performance, for instance - should include a detailed representation of the 
bottom, the geoacoustic model. In practice, one posits a "reasonable" ocean bottom model, and then one proceeds to solve the propagation problem at hand, (the "Direct" problem). Here, the opposite perspective is adopted: Since the ocean bottom affects acoustic wave propagation, would it not be possible to learn something about the bottom from that interaction? (The "Inverse" problem.)

The answer to this question is being sought in the context of an original, single frequency, deep ocean bottom interaction experiment desiqned by G. Frisk and his colleagues of the Woods Hole Oceanographic Institution, and performed in the Hatteras Abyssal Plain(2),(3) and at other locations(4). The monochromatic character of the Frisk method sets it apart from currently used techniques using explosive (or impulse-like) wide-band sources. The Frisk experiment started off as a heuristic approach.

This thesis presents the theoretical basis and numerical analysis of the monochromatic experiment based on an extension of the Gelfand-Levitan theory of quantum scattering. We succeeded in applying a numerical solution to the exact inverse method which distinguishes this solution method from the currently used approximate or trial and error inverse methods. 


\subsection{The Experiment}

The geometry of the experiment is best explained with reference to Fiq. 1. In brief, a driftinq vessel tows a $220 \mathrm{~Hz}$ pulsed CW source. Hydrophones moored close to the bottom record the resultina pressure, both amplitude and phase (via coherent quadrature demodulation), as the ship opens range.

The pulsing of the $220 \mathrm{~Hz}$ source, turning it on for 4 sec every 14 sec, allows for steady state conditions to be attained before any reflections from the ocean surface can reach the receivers. Note that a 14 second duty cycle siqnifies that the acoustic field is sampled spatially once every half wavelength.

The source aperture is small enough compared to the wavelength $(7 \mathrm{~m})$ to be considered an omnidirectional point source. The recorded complex pressure is therefore the field due to the reflections of a spherical wave off the bottom. The information is translated via a Hankel transform, into the plane wave reflection coefficient at a sinale frequency $(220 \mathrm{~Hz})$ for all angles of incidence, both real and complex (5). The critical point to observe is that for a monochromatic plane wave incident on a flat layered bottom, the reflection coefficient is a function of the angle of incidence. At a given angle of incidence, the magnitude and phase of the reflection coefficient depend on the acoustic 


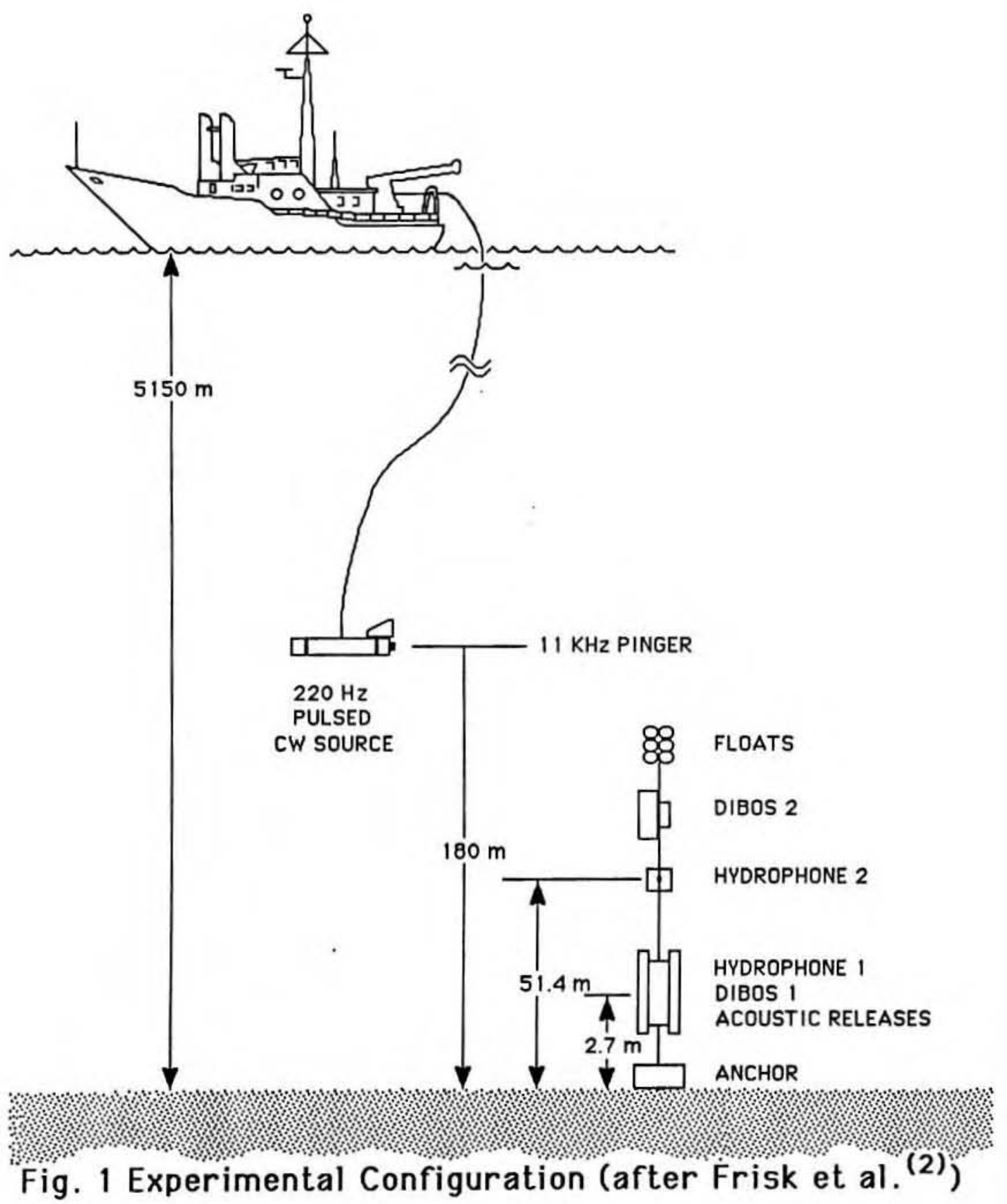

$-10-$ 
properties of the bottom sediments. That naturally leads to the ocean bottom inverse problem: Can the sediment acoustic parameters, velocity and density, be reconstructed from the plane wave reflection data at a sinqle frequency for all anqles of incidence? The thesis aims to elucidate that questions.

\subsection{Experimental Data}

The relationship between the experimental data and the plane wave reflection coefficient has been studied numerically by Frisk et $\mathrm{al}^{(3)}$ and Mook $(6)$. Before beginning our analysis, it is useful here to take a quick look at the underlying theory relating the reflections of spherical waves from a point source, as in the Frisk experiment, to the plane wave reflection as in our model.

The measurements yield the pressure field due to a point source above the bottom half space. Because of the cylindrical symmetry of the problem, the reflected pressure field can be written as a superposition of plane waves

$$
P_{R}(r, z)=\int_{0}^{\infty} \frac{1}{\sqrt{k_{0}^{2}-k_{r}^{2}}} r\left(k_{r}\right) e^{i \sqrt{k_{0}^{2}-k_{r}^{2}}\left(z+z_{0}\right)} J_{0}\left(k_{r} r\right) k_{r} d k_{r}
$$

where $k_{r}$ is the horizontal wavenumber, and $r\left(k_{r}\right)$ is the corresponding reflection coefficient. 
This Sommerfeld integral is a Hankel transform which can be inverted to obtain the reflection coefficient

$$
\Gamma\left(k_{r}\right)=-i \sqrt{k_{0}^{2}-k_{r}^{2}} e^{-i \sqrt{k_{0}^{2}-k_{r}^{2}} \mid z+z_{0}} \mid \int_{0}^{\infty} P_{R}(r) J_{0}\left(k_{r} r\right) d r
$$

Note that $\Gamma\left(k_{r}\right)$ is a function of horizontal wavenumber while the required input to the inverse procedure is the reflection coefficient as a function of the vertical wavenumber $r\left(k_{z}\right)$. The two are of course related by the dispersion relation

$$
\mathrm{k}_{\mathrm{z}}^{2}=\mathrm{k}_{0}^{2}-\mathrm{k}_{\mathrm{r}}^{2}
$$

Given $\Gamma(k r)$ for real $k_{r}$, one can readily generate $r\left(k_{z}\right)$ for $0<\mathrm{k}_{\mathrm{z}}<\mathrm{k}_{0}$. It is more difficult to obtain $r\left(\mathrm{k}_{\mathrm{z}}\right)$ for the full range $0<k_{z}<\infty$ since $k_{0}<k_{z}<\infty$ correspond to $\mathrm{r}\left(k_{r}\right)$ for imaginary $k_{r}$. One approach has been suggested by Stickler (7) and involves the use of a theorem by Van Winter to generate $r\left(k_{z}\right)$ on a ray in the complex plane given its value on the real axis segment $0<k_{z}<k_{0}$. The effect of limiting $r\left(k_{z}\right)$ to real angles $\left(0<k_{z}<k_{0}\right)$ on the inversion for the unknown potential $V(z)$ will be discussed in Chapter VI. 


\subsection{The Model}

A number of assumptions are implicit in the "exact" inverse procedure detailed in the following chapters.

(a) The ocean is assumed homogeneous, and acoustically transparent. In particular, the depth variation of the velocity of sound propagation is neglected. That is a reasonable assumption at the great depth $(5 \mathrm{~km})$ in which the experiment is conducted.

(b) The ocean bottom is assumed to have no horizontal structure, the velocity variation is therefore solely a function of depth. That is a severe restriction imposed by all "exact" inversion formalisms developed to date. Surprisingly, horizontal stratification describes adequately vast areas of the deep ocean floor known as Abyssal plains: These are widespread in the Atlantic and Indian Oceans and in the marginal seas.

The early deep ocean bottom interaction experiments were conducted in the Hatteras Abyssal Plain. This nearly level plain lies at the base of the East Coast Continental rise, and is $1000 \mathrm{~km}$ long by $150-300 \mathrm{~km}$ wide. Its thick (> $1 \mathrm{~km}$ ) sediments were formed by the smooth accumulation of turbidites over the rough basement resulting in one of the flattest areas on earth with slopes of less than $1 \mathrm{~m} / \mathrm{km}$. 
(c) The ocean bottom has been traditionally treated as a fluid in problems involving bottom reflection. In the deep ocean bottom interaction experiments, the acoustic source qenerates compressional (P) waves in the water, which upon propagation in the bottom, a vertically heterogeneous medium, may be converted to shear (SV) waves. The conversion to shear waves will occur discretely at layer interfaces and continuously, where velocity gradients occur. Fryer had shown in one of his papers ${ }^{(8)}$ that coupling for continuously varyinq elastic parameters is neqliqible at frequencies above $20 \mathrm{~Hz}$. Vidmar and Foreman (9) estimated that gradient-induced coupling should be expected in marine sediment at frequencies up to $3 \mathrm{~Hz}$. Another paper by Fryer(10) established that this coupling is extremely small above $1 \mathrm{~Hz}$, regardless of sediment thickness. The most important effect of coupling appears to be the conversion of shear to compressional motion at the sediment basement interface. Note, that although these results are based on a continuously varying structure (approximated by homogeneous layers), they do provide for the sharp discontinuity in elastic parameters at the sediment basement interface. These results do justify the neglect of shear wave effects at the $220 \mathrm{~Hz}$ frequency selected for the experiments that have already been conducted, and at the lower frequencies envisaqed by the Frisk group for future experiments. 
1.5 Overview of the Thesis

In Chapter II, we begin with a mathematical statement of the inverse problem and represent it as a scattering problem for the Schrodinqer equation. We conclude the chapter with a review of the relevant literature.

The input to the inverse procedure, the plane-wave reflection coefficient, and particularly its asymptotic behavior for large vertical wavenumbers are the subject of Chapter III.

Chapter IV presents an extension of the Gelfand-Levitan inversion method to the case of non-zero terminal potential. It is this formulation that permits us an exact solution of the inverse problem so that what remains is the numerical solution of the inteqral equation characterizing the solution.

The derivation presented in Chapter IV is followed in Chapter $\mathrm{V}$ by a discussion of three numerical methods to solve the Gelfand-Levitan integral equation: An improved Born approximation and two finite-difference methods.

The numerical methods outlined in Chapter $\mathrm{V}$ were tested on various postulated acoustic profiles using synthetically generated reflection coefficients. The representative numerical results, the impact of sampling, finite angle aperture, density, loss and noise are discussed in Chapter VI.

Chapter VII comprises the conclusion and suggestions for future work. 
The determination of the acoustic properties of the ocean bottom from the monochromatic plane wave reflection coefficient at all angles of incidence is now shown to be related to a class of inverse problems in quantum scattering theory where an unknown potential in Schrodinger's equation is sought from scattering data. The first part of the chapter casts the problem into mathematical form based on the assumptions set forth in Chapter I. This is followed by a review of the relevant inverse problem literature.

\subsection{Problem Formulation}

(a) Acoustic Wave Equation

In acoustics, the pressure gradient gives rise to an acceleration of mass density $\rho$ according to

$$
\rho \frac{\partial \vec{v}}{\partial t}=-\nabla p
$$

where $p$ is acoustic pressure and $\vec{v}$ is particle velocity. Mass conservation, together with a constitutive relation (Hooke's law), yields:

$$
\frac{\partial p}{\partial t}=-\rho c^{2} \nabla \cdot \vec{v}
$$

in which $\mathrm{c}$ is sound velocity in the medium. 
Fourier transforming the time dependence in the fundamental equations $(2.1)$ and $(2.2),\left[\frac{\partial \omega}{\partial t} \rightarrow-i \omega\right]$, and combining the resulting time-independent equations leads to the acoustic wave equation:

$$
\rho(z) \nabla \cdot\left(\frac{1}{\rho(z)} \nabla p(x, z)\right)+k_{o}^{2} n^{2}(z) p(x, z)=0
$$

with $\mathrm{k}_{\mathrm{O}}=\frac{\omega}{\mathrm{c}_{\mathrm{o}}}$ and index of refraction $\mathrm{n}(\mathrm{z})=\frac{\mathrm{c}_{\mathrm{o}}}{\mathrm{c}(\mathrm{z})} \cdot$ In the derivation of equation (2.3), the acoustic medium has been assumed to be vertically inhomogeneous (or horizontally stratified). In other words, the material properties are a function of depth ( $z$ ) only.

The neglect of density variations reduces equation (2.3) to the Helmholtz equation for the pressure,

$$
\nabla^{2} p(x, z)+k_{0}^{2} n^{2}(z) p(x, z)=0
$$

The equation (2.4) constitutes the starting point of this study

Note: In the presence of smooth density variations, the acoustic equation $(2.3)$ can also be reduced to the Helmholtz equation through the change of variable(11) 


$$
\begin{aligned}
& P=p / \sqrt{\rho} \cdot \quad \text { It follows that, } \\
& \nabla^{2} P+k_{0}{ }^{2} n^{\prime}{ }^{2}(z) P=0
\end{aligned}
$$

with

$$
n^{\prime 2}=n^{2}+k_{o}^{-2}\left(\frac{1}{2 \rho} \nabla_{\rho}^{2}-\frac{3}{4}\left(\frac{1}{\rho} \nabla p\right)^{2}\right) .
$$

(b) Mapping the Seabed Below a Homogeneous Ocean

The specific problem of interest is mapping the seabed below a homogenous ocean. The starting point is again the acoustic wave equation $(2.4)$ :

$$
\nabla^{2} p(x, z)+k_{0}{ }^{2} n^{2}(z) p(x, z)=0
$$

for the configuration shown in Fig. 2. Let

$$
n^{2}(z)=1+\mu^{2}(z) \text {. }
$$

Since the medium is homogeneous in $x$, the spatial variables can be separated by assuming that:

$$
p(x, z, k)=u(z, k) e^{i\left(k_{0} \sin \theta\right) x}
$$

Note that $k_{0} \sin \theta \equiv k_{x}$, the horizontal wavenumber (cf. Fig. 3). 


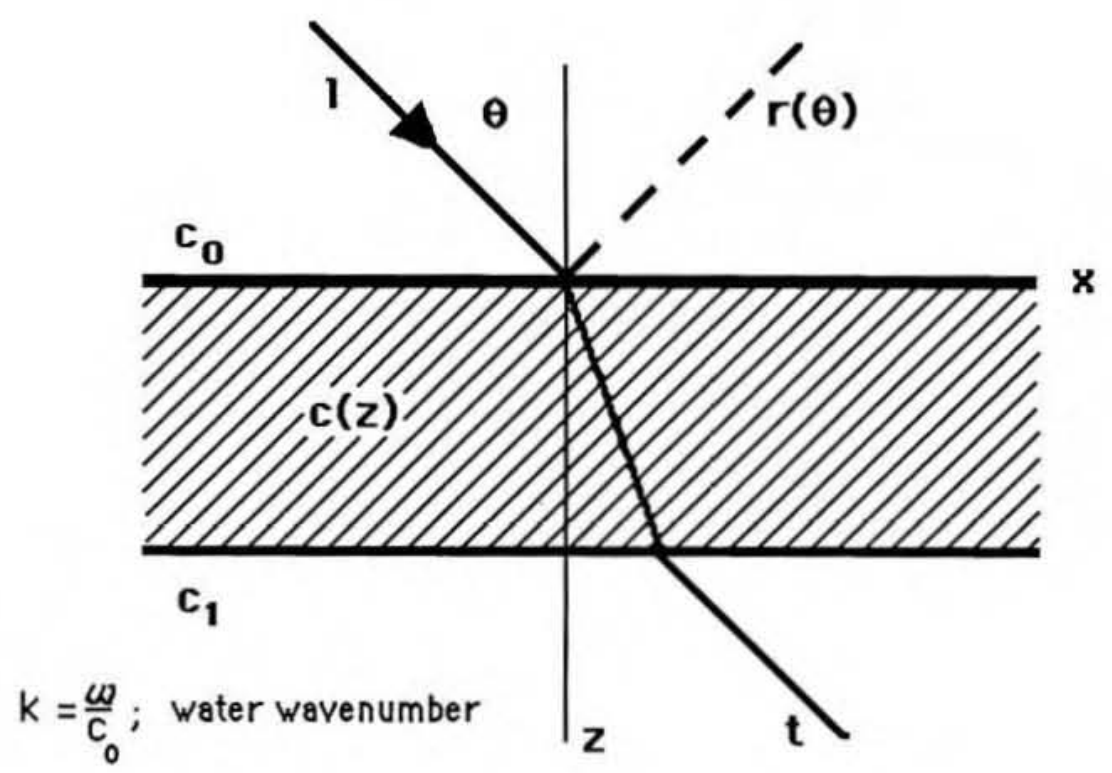

Fig. 2 Schematic Illustration of the Scattering Problem 


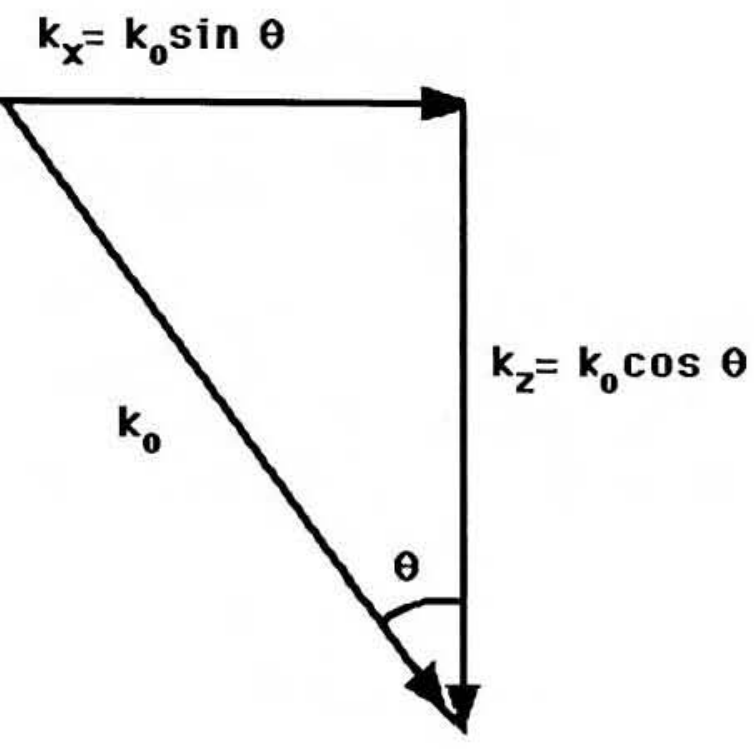

Fig. 3 Wavenumber Decomposition 
Substituting from $(2.7)$ for $p(x, z)$ into wave equation $(2.6)$, one obtains an equation for the $z$ variation of pressure, $u(z)$ :

$$
\left[\frac{d^{2}}{d z^{2}}+\left(k_{0}^{2}-k_{0}^{2} \sin ^{2} \theta\right)+k_{0}^{2} \mu^{2}(z)\right] u=0
$$

or

$$
\frac{d^{2} u}{d z^{2}}+\left[k_{0}^{2} \cos ^{2} \theta+k_{0}^{2} \mu^{2}(z)\right] u=0
$$

this is similar to the time-independent Schrodinger equation. Equation (2.9) can be written in the familiar form:

$$
\frac{d^{2} u}{d z^{2}}(z, E)+[E-V(z)] u(z, E)=0
$$

with the identifications:

$$
\begin{array}{ll}
\mathrm{k}_{\mathrm{z}} & =\mathrm{k}_{\mathrm{O}} \cos \theta, \text { the vertical wavenumber is "momentum". } \\
\mathrm{E} & =\mathrm{k}_{0}{ }^{2} \cos ^{2} \theta \text { is the "enerqy". } \\
\mathrm{V}(\mathrm{z}) & =\mathrm{k}_{\mathrm{O}}{ }^{2} \mu^{2}(\mathrm{z}) \text { is the "potential". }
\end{array}
$$

A few remarks are in order:

\footnotetext{
- The vertical wavenumber, $\mathrm{k}_{\mathrm{z}}$, becomes imaginary for $k_{x}>k_{0}$.
} 

- As $k_{z}$ ranges on $\left[k_{0}, \infty\right], k_{x}$ becomes pure imaginary.
- The energy $E=k_{0}{ }^{2} \cos ^{2} \theta$ is always real. For $k_{z}$ real, $E$ is positive.
- The potential $V(z)={ }^{s_{0}}{ }^{2}\left(1-n^{2}(z)\right)$ is in general positive except, possibly, for a low velocity layer at the ocean bottom interface.

By analoqy with quantum mechanics, one can draw a "potential well" diagram (cf. Fig. 4).

The Schrodinger equation (eq. 2.10) has associated with it two asymptotic boundary conditions:

$$
\begin{aligned}
& u(z) \sim \frac{e^{i k} z}{\sqrt{2 \pi}}+r(p) \frac{e^{-i k} z}{\sqrt{2 \pi}} z+\infty \\
& u(z) \sim t(p) \frac{e^{i k} z}{\sqrt{2 \pi}} \quad z \rightarrow \infty
\end{aligned}
$$

where $r\left(k_{z}\right)$ and $t\left(k_{z}\right)$ are identified as reflection and transmission coefficients respectively.

The problem that was proposed in the introduction has now been cast into an equivalent quantum mechanical problem: 


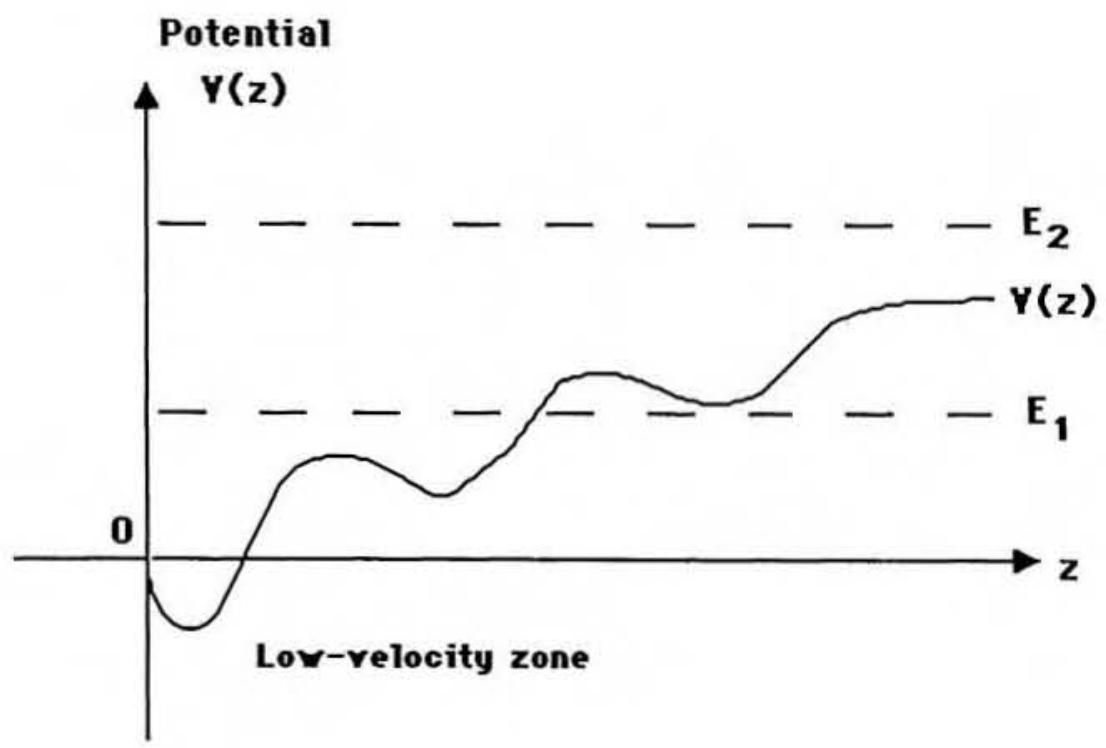

Fig.4 Scattering Potential- Energy Diagram ( $E_{1}, E_{2}$ represent possible Energy levels) 
Given $r\left(k_{z}\right)$, the reflection coefficient, as a function of vertical wavenumber $\mathrm{k}_{\mathrm{z}}$, obtain the scattering potential $\mathrm{V}(\mathrm{z})$ in equation $(2 \cdot 10)$.

\subsection{Survey of Inverse Methods}

\subsubsection{Introduction}

Much of the background methodology relevant to our problem is found in the geophysical literature. An excellent review of the field is provided by Newton $(12,13)$.

The seismic inverse problem for horizontal layered media of infinite depth consists in determining the vertical structure of the acoustic medium (specified usually by impedance, or, in more detail, by density and velocity) from reflection measurements. But for a few exceptions, most of the previous analyses have been confined to excitation with an impulsive pressure signal ( $\delta$-function) and probing at normal incidence. The Fourier transform of a $\delta$-function is essentially flat in frequency domain. What is observed with such an excitation is, therefore, the time trace of the resulting medium resnonse or its Fourier transform. Because of the assumed horizontally layered structure of the medium, and the vertical direction of the siqnal, acoustic properties of the medium change only with depth and thus the problem is one-dimensional. 
In what follows, we present a review of that past inverse problem work that is relevant in several different respects. First, the discussion of the Gelfand-Levitan approach is particularly relevant because this is our basic approach in this thesis adapted to the Frisk experiment.

Next, the discussion of prior work on sinqle frequency excitation, launched at non-normal incidence presents the state of the problem before we addressed it.

The Deift and Trubowitz method is described because Stickler, having been briefed on our work, adapted the Deift and Trubowitz method to the Frisk experiment and was able to devise an alternate approach to its analysis.

The discussion of the Schur algorithm reviews the analysis by Yagle and Levy of probing with an impulsive excitation also at non-normal incidence. We comment on why Yagle and Levy dismissed the Gelfand-Levitan approach as inferior to the Schur algorithm although we have in fact, successfully adapted Gelfand-Levitan to the solution of the monochromatic, non-normal incidence problem.

The Riccati equation method is discussed although it was not used. We considered this approach and believe it to be promising, but this method was not fully explored. 


\subsubsection{The Gelfand-Levitan Approach}

The problem that was proposed at the end of section (2.1), the reconstruction of the potential of a Schrodinger equation from the reflection coefficient, is related to a classic problem of quantum scattering theory: How to reconstruct a Sturm-Liouville differential equation from its spectral function. The rroblem was solved in a celebrated paper by Gelfand and Levitan (14) who, since they were discussing the radial wave equation, were interested only in a solution on the half-line $0<r<\infty$ (standing-wave problem). Subsequent developments (e.q., (15)) led to formulations on the full line $-\infty<z<\infty$ in terms of such readily measured quantities as the phase shift or reflection coefficient (traveling-wave problem). An excellent distillation of these ideas is to be found in the papers of Faddeyev(16), while a more general survey of the field of inverse scatterind has been carried out more recently by Chadan and Sabatier(17). The interrelation between the different approaches and their time-domain interpretation has been presented by Burridae $(8)$. A detailed theoretical presentation and extension of the Gelfand-Levitan theory and its application to our problem will be taken up in Chapter IV.

The exploitation of the Gelfand-Levitan formalism outside of quantum mechanics was first taken up by Kay (19) and Moses and deRidder $(20)$ to solve problems in electromagnetics 
such as the characterization of transmission lines and dielectrics from scattering data. The possibility of monochromatic probing is mentioned briefly but not pursued. An interesting by-product of this research is the theoretical construction of dielectrics which are reflectionless at all frequencies.

The Gelfand-Levitan approach was introduced into the field of seismic exploration by Ware and Aki(21). They presented an analytic approach to the inverse scattering problem for elastic wave propaqation in a stratified medium when the medium is probed with impulsive plane waves at normal incidence. The analytic solution was obtained by transforming the equation of motion in a stratified elastic medium for plane waves at normal incidence into a one-dimensional Schrodinger equation. The potential of the resulting Schrodinger equation depends only on the impedance of the medium as a function of travel time. No ambiguity arises owing to the bound state solutions of the schrodinger equation. Ware and Aki went on to establish a discrete analogy of the continuous solution showing aqain that the impedance of the medium could be recovered as a result of probing at normal incidence when the medium consists of a homogeneous half-space of impedance $\rho_{\circ} C_{O}$ in contact with a sequence of $n$ homoqeneous layers of impedance $\rho_{1} c_{1}, \rho_{2} c_{2}, \ldots, \rho_{n} c_{n}$ and terminates with a homogeneous half-space of impedance $\rho_{n+1} c_{n+1}$. The sequence of $n$ homogeneous layers which have 
thickness $\Delta \eta_{i}$ and which are chosen such that travel time through each layer is a constant,

$$
\Delta n_{i} / c_{i}=\Delta t=\text { constant }
$$

constitutes what is known as a Goupillaud layered medium.

An elegant solution $0:$ the inversion of a Goupillaud medium has been given by claerbout (22) using z-transforms. Ware and Aki showed the equivalence of the Goupillaud solution and of the discretized version of their continuous solution. They had promised a second paper dealing with the inverse scattering problem for plane waves at non-normal incidence. Such a paper was, however, never published. Although Ware had obtained in his thesis some partial results at non-normal incidence prior to the publication of the Ware and Aki paper, the approach in the thesis was too cumbersome and in fact had hit an unsurmountable wall at and above the critical angle: the reflection coefficient tends to one as $\omega+\infty$ and therefore fails to meet an integrability criterion required in the application of the Gelfand-Levitan algorithm.

The question arises as to how our approach, a monochromatic experiment at all angles of incidence, relates to the Ware and Aki experiment of an impulsive broadband source at one angle of incidence? The vertical wavenumbers generated in Ware and Aki (normal incidence) $k_{z}=\frac{\omega}{c_{0}}($ for $0<\omega<\infty)$. Cover the ranqe from 0 to $\infty$ as the frequency is swept. One 
can see from Fig. 5 that in our experiment $k_{z}=k_{0} \cos \theta$ (for $0<\cos \theta<\infty)$ formally $\operatorname{covers}$ the same range of values of $k_{z}$ although the experiment is monochromatic. There is, however, a fundamental difference between the two approaches in that, as we prove in Chapter III, the reflection response in our monochromatic experiment is inteqrable and in fact qoes to zero as $k_{z}$ goes to infinity at least as fast as $\left(1 / \mathrm{k}_{\mathrm{z}}{ }^{2}\right)$. It should be noted that Ware and Aki did not run any computer simulations of their algorithm, and were therefore unaware of its numerical performance (in fact, the Gelfand-Levitan approach was widely held at the time to be numerically unstable).

Inspired by the Ware and Aki approach, a number of researchers particularly Ahn, Jordan, and $\operatorname{Kritikos}(23,24,25)$ applied the Gelfand-Levitan algorithm to the analytical problem of the reconstruction of dielectric functions and electron density profiles. Their work is an analytical attempt to solve the problem when a dielectric medium is probed with impulses at normal incidence. Most of their effort was applied to the closed-form solution of the Gelfand-Levitan equation. Such a solution is possible when the reflection coefficient can be represented as a rational function of wavenumber. Although the approximation of the reflection coefficient by rational functions has not yet received any practical application, the availability of such closed-form solutions provided us with valuable canonical examples against which to check numerical inversion results. 


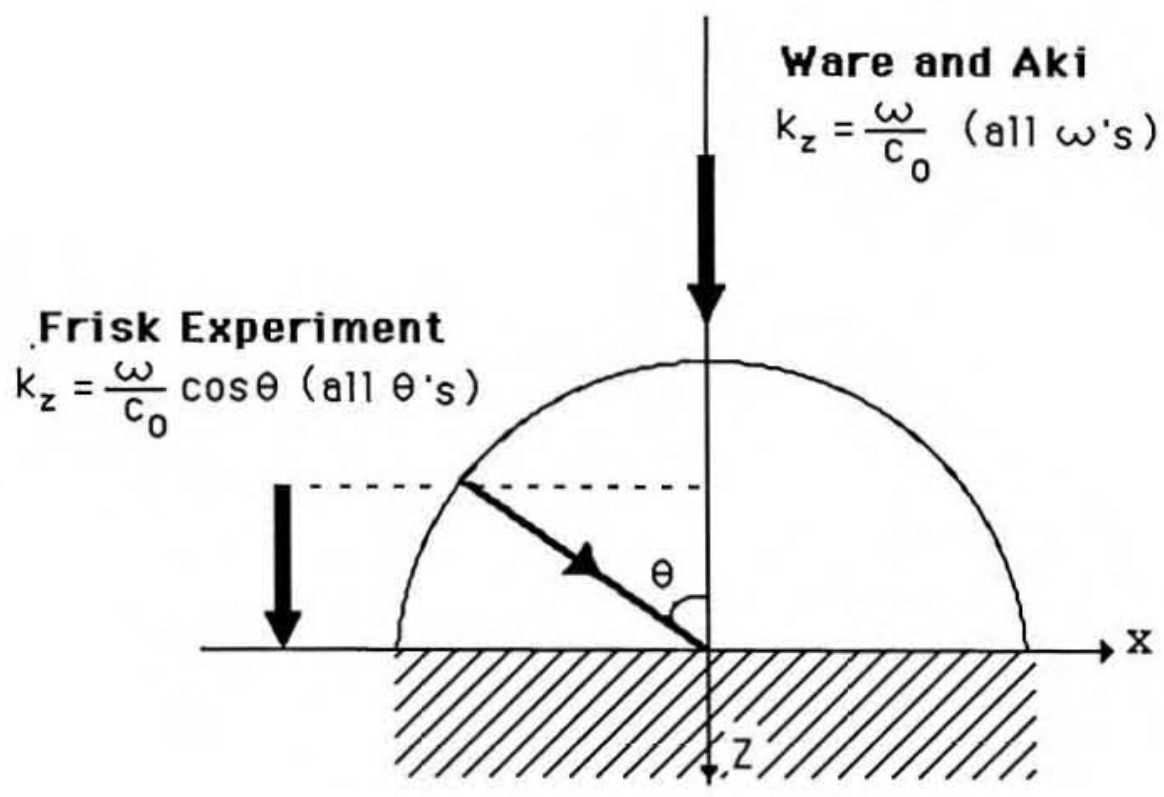

Fig. 5 Generation of Vertical Wavenumbers in the Ware and Aki Method and in the Frisk Experiment 
More recently, Berryman and Greene(26) have addressed some long-standing questions reqarding the general applicability of the Goupillaud method. They demonstrated the equivalence of the Goupillaud method of inversion and of the Marchenko method(15) for the schrodinger equation for models with arbitrary layer thicknesses (i.e., continuous impedance variation). When the reflection coefficients are correctly interpreted, in the continuum limit, both methods will reconstruct the same impedance except, possibly, for the values at a finite number of jump points in any finite span of travel time. As part of this work, Berryman and Greene presented a fast $\left(0\left(\mathrm{~N}^{2}\right)\right)$ recursive alqorithm analogous to the Levinson procedure for the inversion of a Toeplitz matrix. We were able to adapt this algorithm and use it in our numerical computations.

During our research, we became aware of an unpublished report by Jacobs and Stolt (27) which demonstrates four different coordinate transformations which convert the laterally homogeneous acoustic wave equation of the Schrodinger form. One of the transformations takes frequency $\omega$ to be a fixed parameter which infers our monochromatic condition. However, Jacobs and stolt use a slightly different potential function than the one chosen in this thesis. Their effort to verify the Gelfand-Levitan algorithm is similar to the one presented earlier by Moses and deRidder(20). In discussing the Gelfand-Levitan algorithm, 
they make the important assertion that the algorithm holds even for the case of dissimilar end potentials. We have presented, independently, a rigorous proof of that assertion in Chapter IV.

The method of Carroll and Santosa(28) was used by Santosa(29) to solve the inverse problem for an impulsive source at normal incidence. Although the method is similar to the Gelfand-Levitan approach, it is not based on the Schrodinger equation but rather on the equation

$$
\hat{v}^{\prime \prime}+\omega^{2} \hat{v}=q(y) \hat{v}^{\prime}
$$

where $\hat{v}$ is the Fourier transform of the shear displacement and $q(y)$ is related to the chanqe in the impedance $A(y)$ by

$$
q(y)=-\frac{A^{\prime}(y)}{A(y)}
$$

The measured response $g(t)=\delta(t, x=0)$ is transformed into the spectral density $G(\omega)$

$$
G(\omega)=\frac{-2 \omega}{\pi} \int_{0}^{\infty} g(t) \exp (i \omega t) d t .
$$


The Gelfand-Levitan type equation that is to be solved is then

$$
T(y, z)+K(y, z)-\int_{0}^{Y} K(y, n) T_{n}(n, z) d \eta=0, \quad z \leqslant y
$$

where $T(y, z)$ is qiven by

$$
T(y, z)=\int_{0}^{\infty} \frac{\sin \omega z}{\omega} \cos \omega y\left[G(\omega)-\frac{2}{\pi}\right] d \omega
$$

and the impedance profile is recovered from $K(y, x)$ through

$$
a(y)=-\frac{2[K(y, y)]^{\prime}}{[1-K(y, y)]}
$$

The major difference from the standard Gelfand-Levitan procedure is that in (2.15) the kernel $\mathrm{T}$ is differentiated with respect to $n$.

Santosa(30) refined the method to give it a time-domain meaning by applying it to problems in which the response data are given for a finite time. The representation obtained is similar to that in the Gopinath-Sondhi equation(31). Santosa demonstrated the method to be stable both theoretically and numerically on a window type profile. Reconstruction errors 
at depth are attributed to errors in the reflection data and to the first order discretization errors committed in the approximation of an integral by a sum.

Coen has extended in one of his papers $(32)$ the work of Ware and Aki so as to recover both the density and compressibility profiles of a layered fluid from the plane wave reflection coefficient at two precritical angles of incidence, and at all frequencies.

In another paper(33), Coen applied the ware and Aki method to recover the three elastic profiles of a layered half-space from three reflection coefficients. First the shear modulus and density profiles are determined from reflection coefficient data for oblique incidence $\mathrm{sH}$ plane waves qiven at two anqles of incidence and for all frequencies. Once the density and shear modulus have been obtained, a further experiment using the reflection coefficient due to an impulsive normally incident $\mathrm{P}$-wave permits the retrieval of the P-wave velocity and hence of the Lame profile. The limitation in Coen's work, as in Ware and Aki's, is that the potential $V(\zeta)$ satisfy the integrability condition.

$$
\int_{0}^{\infty}(1+|\zeta|)|v(\zeta)| d \zeta<\infty
$$


which would be very restrictive in practice. One result we demonstrate in our work is that the Gelfand-Levitan algorithm still applies to the fundamental case when $V(\zeta)$ tends to a non-zero finite value at infinity in violation of the above intearability condition.

In a third paper( 34$)$, Coen addresses the problem of common source noint surface data wherein a source is placed on the free surface of a plane stratified half-space and the vertical component of velocity or of acceleration is measured on the free surface. After solving the impulsive source problem, Coen discusses the monochromatic source problem. His approach is deceptively similar to the one we present in this thesis as both approaches transform the original problem into a one-dimensional Schrodinger equation and then proceed to use the Gelfand-Levitan inteqral equation to solve the inverse problem. However, the problem in the two approaches is posed in a different way and the steps towards the solution are dissimilar.

It is useful here to run through Coen's method so as to point out the difficulty he encounters and which does not arise in our approach (a constant density is assumed). 
The Hankel transform of the pressure field

$$
\phi(\zeta, z, \omega)=\int_{0}^{\infty} \int_{0}^{\infty} p(r, z, t) r J_{0}(r, \zeta) e^{-i \omega t} d r d t
$$

satisfies the schrodinger equation

$$
\left(\frac{\partial^{2}}{\partial z^{2}}-k^{2}\right) \phi(k, z, \omega)=Q(z, \omega) \phi(k, z, \omega) .
$$

where $Q(z, \omega)$ is the potential

$$
Q(z, \omega)=\frac{\omega^{2}}{c_{0}^{2}}\left(1-\frac{c_{0}^{2}}{c^{2}}\right)
$$

and $\mathrm{k}$ is related to the horizontal wavenumber $\zeta$ through

$$
\zeta=\sqrt{k^{2}+\frac{\omega^{2}}{c_{0}^{2}}} .
$$


The Schrodinger equation is accompanied by two initial conditions

$$
\left.\phi\right|_{z=0}=1,\left.\quad \phi^{\prime}\right|_{z=0}=-\frac{1}{q(\xi, \omega)}
$$

where

$$
g(\xi, \omega)=-\frac{1}{\rho(0) \frac{d}{d}(\xi, \omega)}
$$

and $d(\zeta, \omega)$ is the Hankel transform of the vertical component of particle acceleration at the surface $z=0$.

$$
\text { Coen's scheme proceeds from an input function } r(k, \omega)
$$

$$
r(k, \omega)=\frac{1-k q(k, \omega)}{1+k q(k, \omega)}
$$

given for all real positive $\mathrm{k}$ values and requires the computation of $R(z, w)$ where

$$
r(k, \omega)=\int_{0}^{\infty} R(z, \omega) e^{-k z} d z \quad .
$$


The Gelfand-Levitan equation

$$
A(z, y, \omega)=R(z+y, \omega)+\int_{-z}^{z} A(z, x, \omega) R(y+x) d y|y|<z
$$

is then solved for $A(z, y)$ which in turn yields the potential

$$
Q(z)=2 \frac{d}{d z} A(z, z), \quad z>0 .
$$

The difficulty with the whole procedure stems from the second step, namely the computation of $R(z)$ from $r(k)$. That involves an inverse Laplace transform which is numerically inherently unstable. Our method, on the other hand, starts off from the Schrodinger equation for the field (rather than for its Hankel transform) with the associated plane wave reflection coefficient as a function of vertical wavenumber. The Laplace transform of Coen's approach is replaced by a Fourier transform which does not present any numerical difficulties. It is to be noted that the known numerical instability of the Laplace transform has led some researchers, notably Santosa and Symes(42) to dismiss the Gelfand-Levitan approach to the solution of the inverse problem. We believe that our approach to the inverse problem could lead to a positive reassessment of the Gelfand-Levitan inverse method. 


\subsubsection{Single Frequency, Non-Normal Incidence}

Very few researchers other than Coen have considered the single frequency non-normal incidence case. One exception is Mittra and Schaubert(35) who used a method different from ours. Their approach is a spectral domain method of probing stratified, lossless, dielectric media using an alternative to the Marchenko formulation and resulting in a Fredholm equation of the second kind which is solved through the use of rational basis functions. They noted that accurate inversions can be obtained if data is provided for $k_{z} \gg k_{0}$. The Mittra and Schaubert examples all have zero terminal potentials, and although the results are good in general, the inaccuracies are interestingly larger near the origin with higher frequencies "seeming to give better resolution."(35)

Another example of the prior single frequency, nonnormal incidence analysis is provided by the work of Roger $(36)$. Roger sought to determine the index profile of a dielectric plate backed by a perfectly conducting plane. That last fact complicates the problem, since the potential is always negative and bound states due to surface waves might exist. Roger starts from a nonlinear integral equation which he linearizes to obtain a Fredholm equation of the first kind whose solution constitutes an ill-posed problem (in the sense of Hadamard). Roger solves this equation by using the Tikhonov regularization method. The method fails when the permittivity $\varepsilon(z)$ exceeds a constant by more than $20 \%$ and also when the layer is thicker than $1.5 \lambda$. 


\subsubsection{The Deift and Trubowitz Method}

Deift and Trubowitz (37) introduced the trace method for determining the potential in one-dimensional scattering problems for the Schrodinger equation. The trace method requires as data the reflection coefficient, while the method we adopted requires the Fourier transform of the reflection coefficient. Furthermore, the trace method requires the solution of a nonlinear differential equation while the Gelfand-Levitan (or Marchenko) equation that we use is a linear integral equation.

Stickler visited us in Woods Hole and became interested in adapting the Deift and Trubowitz trace method to our problem. He took the same input, i.e., the measurement of the pressure field as a function of range, where both the real and imaginary parts of the pressure field are needed. After the reflection coefficient $R(k)$ is derived by using the same approach as ours, he then introduces an auxiliary potential, $\tilde{q}(z)$, which is determined by using the trace formula methods of Deift and Trubowitz. Stickler(7) defines the auxiliary potential, $\tilde{q}(z)$ by

$$
\tilde{q}(z)=q(z)-\frac{\sqrt{\rho}}{2}\left(\frac{\rho^{\prime}}{\rho / 2}\right){ }^{\prime}
$$

where primes denote derivatives with respect to $z$. The auxiliary potential, $\tilde{q}(z)$, can be determined from the Deift and Trubowitz trace formula 


$$
\tilde{\mathrm{q}}(\mathrm{z})=-\frac{4}{\pi} \int_{0}^{\infty} k \operatorname{Im}\left[R(k) u_{2}^{2}(z,-k)\right] d k .
$$

The Jost function $u_{2}(z, k)$ in $(2.30)$ is determined by solving

$$
-u_{2}^{\prime \prime}+\tilde{q}(z) u_{2}=k^{2} u_{2}
$$

with the boundary condition

$$
u_{2} \sim e^{-i k z}, z+\infty .
$$

Deift and Trubowitz have shown that the usual iteration scheme for solving two coupled nonlinear integral equations such as (2.13) and (2.14) converges. In our case, instead of (2.13) and (2.14), we solve for $\tilde{q}(z)$ using a Gelfand-Levitan linear integral equation.

Stickler presented two numerical examples(7) of applying the Deift and Trubowitz algorithm on a twice continuous function (in Chapter VI we apply our method to one of his examples and refer to it as the "Stickler's Profile"). Since Stickler generated the reflection coefficient from the solution of a Riccati equation, he had control over the local tolerance for the determination of the reflection coefficient. As in the Gelfand-Levitan method, the results are excellent for $\mathrm{z} / \mathrm{L} \ll 1$, but deteriorate gradually with depth $(z / L>1)$. Stickler attributes the degradation of his method 
to the lack of accuracy of the local reflection coefficients generated via the Riccati equation.

When we compare Stickler's numerical results to ours, we observe the general similarity of his results to the ones described in this thesis. This similarity should not be too surprising in view of the close relationship between the two methods (see further discussion in Chapter IV). We have no data to assess the computational efficiency of Stickler's method versus ours.

\subsubsection{Schur Algorithm}

Yagle and Levy $(38,39)$ have adopted an algorithm which reconstructs the unknown acoustic medium layer by layer (layer stripping procedure). The method is analogous to the downward continuation method, in that successive up and downgoing waves are measured at the surface. The first reflection of the impulse yields information about the medium immediately beneath the surface (at depth $\Delta$ ). This information is used to update the waves at depth $\Delta$ which then becomes the new reference surface. The procedure is successively repeated until the depth of interest is reached. The Schur algorithm applies to the study of the two component system of coupled differential equations

$$
\begin{aligned}
& q_{1 x}(x, t)+q_{1 t}(x, t)=-r(x) q_{2}(x, t) \\
& q_{2 x}(x, t)-q_{2 t}(x, t)=-r(x) q_{1}(x, t)
\end{aligned}
$$


where the subscripts $\mathrm{x}$ and $\mathrm{t}$ denote derivatives with respect to $x$ and $t$, and $r(x)$, the reflectivity function, provides a coupling between the downgoing wave $q_{1}(x, t)$ and the upgoing wave $q_{2}(x, t)$ (unit velocity).

Yagle and Levy begin their derivation with the set of equations arising after an initial impulse excitation $\delta(t)$, so that $q_{1}(x, t)$ and $q_{2}(x, t)$ can be written as

$$
\begin{aligned}
& q_{1}(x, t)=\delta(t-u)+\tilde{q}_{1}(x, t) u_{-1}(t-u) \\
& q_{2}(x, t)=\tilde{q}_{2}(x, t) u_{-1}(t-u),
\end{aligned}
$$

in which causality has been used (no waves exist for $t<u$ ). From (2.33) and (2.34) Yagle and Levy derive

$$
r(x)=2 q_{2}(u, x)
$$

The equations (2.33) and (2.35) constitute the continuous parameter fast Cholesky recursion where $q_{1}(x, t)$ and $q_{2}(x, t)$ are updated to yield $r(x)$ from equation (2.35).

At this point, the application of the Schur method entails taking the Fourier transforms of the system in (2.33). Denoting the transform of $q$ by $\hat{q}$, we get:

$$
\begin{aligned}
& \hat{\mathrm{q}}_{1 x}=-i \omega \hat{\mathrm{q}}_{1}(x, \omega)-r(x) \hat{\mathrm{q}}_{2}(x, \omega) \\
& \hat{\mathrm{q}}_{2 x}=-r(x) \hat{\mathrm{q}}_{1}(x, \omega)+i \omega \hat{\mathrm{q}}_{2}(x, \omega) .
\end{aligned}
$$

Yagle and Levy thus find a reflection coefficient 


$$
\hat{R}(x, w)=\frac{\hat{q}_{2}(x, \omega)}{\hat{q}_{1}(x, \omega)}
$$

which obeys a Riccati equation

$$
\hat{R}_{x}=2 i \omega \hat{R}(x, \omega)+r(x)\left(\hat{R}(x, \omega)^{2}-1\right)
$$

with

$$
r(x)=\lim _{\omega \rightarrow \infty}[2 i w \hat{R}(x, \omega)]
$$

Equations $(2.36),(2.37)$, and (2.38) constitute the Schur algorithm, while (2.38) and (2.39) represent a continuous parameter dynamic deconvolution algorithm. It is to be noted that the discretized Schur algorithm is similar to the fast recursion procedure of Berryman and Greene.

Yagle and Levy assert in the concluding section of their paper that their Schur algorithm is computationally superior to the Gelfand-Levitan algorithm as used by Coen. This observation may be true of the Gelfand-Levitan procedure for impulsive sources at non-normal incidence as presented by Ware and $\mathrm{Aki}^{(21)}$ and by Coen(33), but it certainly does not apply to our approach. Yagle and Levy's main objection to Gelfand-Levitan is that the boundedness of the potential 


$$
\int_{0}^{\infty}(1+|\tau|)|v(\tau)| d \tau<\infty
$$

may not be satisfied at non-normal incidence. However, we show in Chapter IV, that the Gelfand-Levitan algorithm can in fact be applied even for a non-zero end potential which would render the integral in (2.40) infinite. Moreover, the Frisk experiment studied here is performed at a single frequency and not with an impulsive excitation. We have shown that the monochromatic reflection coefficient as a function of vertical wave number is integrable so that the negative comment of Yagle and Levy does not apply to our work.

Although not stated in their paper, the application of the Schur algorithm to the inverse problem in a layered acoustic medium involves implicitly approximations similar to those inherent in the Claerbout's migration method. Although the Schur algorithm constitutes an improvement over migration in so far as the downgoing wave strength is modified by the upgoing wave strength it is still an approximation. Indeed, in some of our earlier unpublished work, we succeeded in improving the claerbout migration method precisely by introducing the coupling between the reflected and the downgoing wave. By contrast, our Gelfand-Levitan approach is not an approximation and except for numerical computation represents an exact formulation of the problem. 


\subsubsection{Riccati Equation Method}

One method which we considered upon Stickler's suggestion and which appears to have considerable potential is based on the Riccati equation. Reflectivity as a function of travel time $\tau$ obeys the Riccati equation(12)

$$
\frac{d r}{d \tau}=-2 i \omega r-\gamma(\tau)\left(1-r^{2}\right)
$$

with boundary condition $r(\omega, \infty)=0$ in which

$$
\gamma(\tau)=\frac{1}{Z} \frac{d z}{d \tau}
$$

where the acoustic impedance has been defined by

$$
Z(\tau)=\sqrt{\frac{\omega}{k_{z} \rho}}
$$

The inverse problem is here that of reconstructing $\mathrm{Z}(\mathrm{z})$ from surface observations of $r$. To get $z(z)$ from $z(\tau)$ involves further assumptions. We can formulate the problem equivalently by writing

$$
r(\omega, \tau)=\int_{\tau}^{\infty} d \tau^{\prime} \gamma\left(\tau^{\prime}\right) e^{\left[2 i \omega\left(\tau^{\prime}-\tau\right)\right]}\left(1-r^{2}(\omega, \tau)\right)
$$

while at the surface, the reflection amplitude is

$$
r(\omega, 0)=\int_{0}^{\infty} d \tau \gamma(\tau) e^{(i \omega \tau)}\left(1-r^{2}(\omega, \tau)\right)
$$


The last two equations can be regarded as a nonlinear mapping of $r(\omega, 0)$, given as a function of $\omega$, into $\gamma(\tau)$. The aim of such an approach would be to reconstruct $\gamma(\tau)$ from $r(\omega, 0)$. Although the existence of a complete solution to the above problem has not yet been demonstrated, an approximation can be derived. An inverse Fourier transform of (2.28) yields

$$
\begin{aligned}
r(t)=\frac{1}{\pi} & \int_{-\infty}^{\infty} \mathrm{d} \omega \mathrm{e}^{-(2 i \omega \tau)} r(\omega, 0) \\
& +\frac{1}{\pi} \int_{-\infty}^{\infty} \mathrm{d} \omega \int_{0}^{\infty} \mathrm{d} \tau^{\prime} \alpha\left(\tau^{\prime}\right) r^{2}\left(\omega, \tau^{\prime}\right) x \mathrm{e}^{\left[2 i \omega\left(\tau^{\prime}-\tau\right)\right]} .
\end{aligned}
$$

The first approximation to the solution is the Born series term, namely

$$
\gamma_{1}(\tau)=\frac{1}{\pi} \int_{-\infty}^{\infty} d \omega r(\omega, 0) e^{-2 i \omega \tau}
$$

which resembles the first term of our own result. The convergence of the iteration series solution has not yet been established, but the approach as formulated by Nilsen and Gjevik(40) appears promising. 
It is useful here to note that a Riccati equation still applies in the presence of density discontinuities. The equation obeyed by the pressure $p$ is

$$
\rho\left(\rho^{-1} p^{\prime}\right)^{\prime}+\left(k^{2}-v\right) p=0,
$$

where $\mathrm{V}$ is the potential.

The reflection coefficient may be obtained at the surface from the continuity of pressure and vertical velocity.

$$
\left.R(k)\right|_{z=0}=\frac{\rho^{-1} p^{\prime}-i k p}{-i k}
$$

The ratio of vertical velocity to pressure, i.e., the admittance,

$$
u=\frac{p^{-1} p^{\prime}}{p}
$$

is continuous even in the presence of material discontinuities, as it is the ratio of two continuous quantities. The admittance u obeys a Riccati equation, 


$$
u^{\prime}=-Q-\rho u^{2}
$$

in which $Q=\frac{1}{\rho}\left(k^{2}-v\right)$

Note that $u(z=0)=i k \frac{(1-R)}{1+R}$

Let $u(z)=i k \frac{(1-w(z))}{1+W(z)}$

It follows that $w(0)=\left.R(k)\right|_{z=0}$, and $w(z)$ itself obeys a Riccati equation

$$
2 i k W^{\prime}-2 W\left(Q+\rho k^{2}\right)+W^{-2}\left(\rho k^{2}-Q\right)=\left(\Omega-\rho k^{2}\right)
$$

which can be used to generate the reflection coefficient in the direct problem or to use an iterative procedure for the inverse problem. We did not pursue this approach further but it merits further study.

We have covered here those papers that were most relevant to the work that follows. It should be noted, how- 
ever, that there are a number of other interesting inverse methods that have not been included in this review. Particularly noteworthy are the papers by Moses(41), Gopinath and Sondhi(31), Santosa and Symes $(42)$ and others $(43,44)$. Also of interest is additional literature on the approximate inverse methods ${ }^{(55-57)}$. Special mention should be made of methods based on the Born approximation which originated with the seminal work of Cohen and Bleistein ${ }^{(45-54)}$. 


\section{THE REFLECTION COEFFICIENT}

\subsection{Introduction}

The plane-wave reflection coefficient as a function of vertical wavenumber $r\left(k_{z}\right)$ is central to the inversion procedure we adopted to solve the inverse problem. Its symmetry property is shown to follow from the integral equation representation of the field. We then derive its asymptotic behavior for large $\mathrm{k}_{\mathrm{z}}$ by induction and show that $r\left(\mathrm{k}_{z}\right)$ is integrable, and hence is an acceptable input to the inverse method presented in Chapter IV.

\subsection{Definition and Properties of the Reflection Coefficient} Consider the problem of a plane wave

$$
\psi_{i}(z)=e^{i k z}
$$

incident from $z=-\infty$ onto a half-space extending from $z=0$ to $z=+\infty$. The half space is characterized by a potential $\mathrm{V}(\mathrm{z})$. $\mathrm{V}(\mathrm{z})$ is all that is needed to describe the scattering of the incident wave $\psi_{i}(z)$ by the acoustic half space:

$$
\psi(z)=e^{i k z^{z}}+\int_{-\infty}^{\infty} G\left(z, z^{\prime}\right) V\left(z^{\prime}\right) \psi\left(z^{\prime}\right) d z^{\prime}-\infty<z<\infty
$$


where the Green's function $G\left(z, z^{\prime}\right)$ is determined by

$$
G\left(z, z^{\prime}\right)=\frac{e^{i k_{z}\left|z-z^{\prime}\right|}}{2 i k_{z}}
$$

It follows that as $z \rightarrow-\infty$,

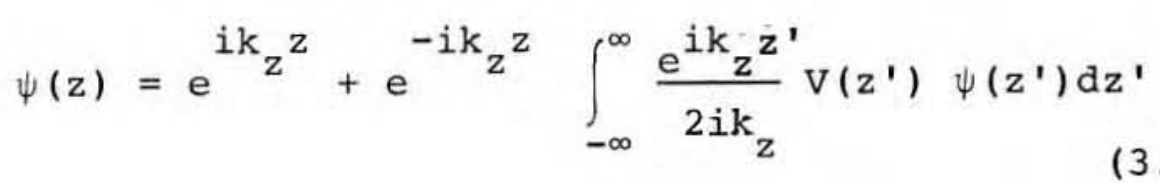

and the coefficient of $e^{i k z z}$ can be identified as the reflection coefficient.

$$
r\left(k_{z}\right)=\frac{1}{2 i k_{z}} \int_{-\infty}^{\infty} e^{+i k z^{\prime}} v\left(z^{\prime}\right) \psi\left(z^{\prime}\right) d z^{\prime}
$$

The integral representation of the reflection coefficient allows a simple derivation of the symmetry properties of $r\left(k_{z}\right)$ in the complex plane.

From (3.4)

$$
r\left(-k_{z}\right)=-\frac{1}{2 i k_{z}} \int_{-\infty}^{\infty} e^{-i k_{z^{\prime}}{ }^{\prime}} V\left(z^{\prime}\right) \psi\left(z^{\prime},-k_{z}\right) d z^{\prime}
$$

But, for a real potential $\mathrm{V}(\mathrm{z})$ and for real $\mathrm{k}_{\mathrm{z}}$, Schrodinger's equation shows that when $\psi\left(z^{\prime}, k_{z}\right)$ is a solution, so is $\psi\left(z^{\prime},-k_{z}\right)$. Moreover, $\psi\left(z^{\prime},-k_{z}\right)=\psi *\left(z^{\prime}, k_{z}\right)$ which implies 


$$
r\left(-k_{z}\right)=r *\left(k_{z}\right)
$$

The result can be extended to the complex plane through the use of the Schwartz reflection principle,

$$
r\left(-k_{z}^{*}\right)=r^{*}\left(k_{z}\right)
$$

in any region of analyticity connected with the real $k_{z}$ axis. (This constitutes the analytic continuation of $r\left(k_{z}\right)$ ). Now, the Gelfand-Levitan algorithm requires knowledge of $r\left(k_{z}\right)$ for all real $k_{z}$. However, the symmetry property expressed in (equation 3.6) demonstrates that knowledge of $r\left(k_{z}\right)$ on the half-line of $0<k_{z}<\infty$ is sufficient.

Note that when the vertical wavenumber $\mathrm{k}_{\mathrm{z}}=\mathrm{k}_{0} \cos \theta$ is real and larger than the water wavenumber $\mathrm{k}_{0}$, the angle of incidence becomes imaginary. That can be verified by requiring that $\cos \theta=\cos \left(\theta_{r}+i \theta_{i}\right)$ be real. And since

$$
\cos \left(\theta_{r}+i \theta_{i}\right)=\cos \theta_{r} \cosh \theta_{i}-i \sin \theta_{r} \sinh \theta_{i}
$$

it follows that $\theta_{r}=0$ and $k_{z}=k_{0} \cosh \theta_{i}$ for $k_{0}<k_{z}<\infty$. The mapping between the $k_{z}$-plane and the $\theta$-plane is drawn in Figure 6. 


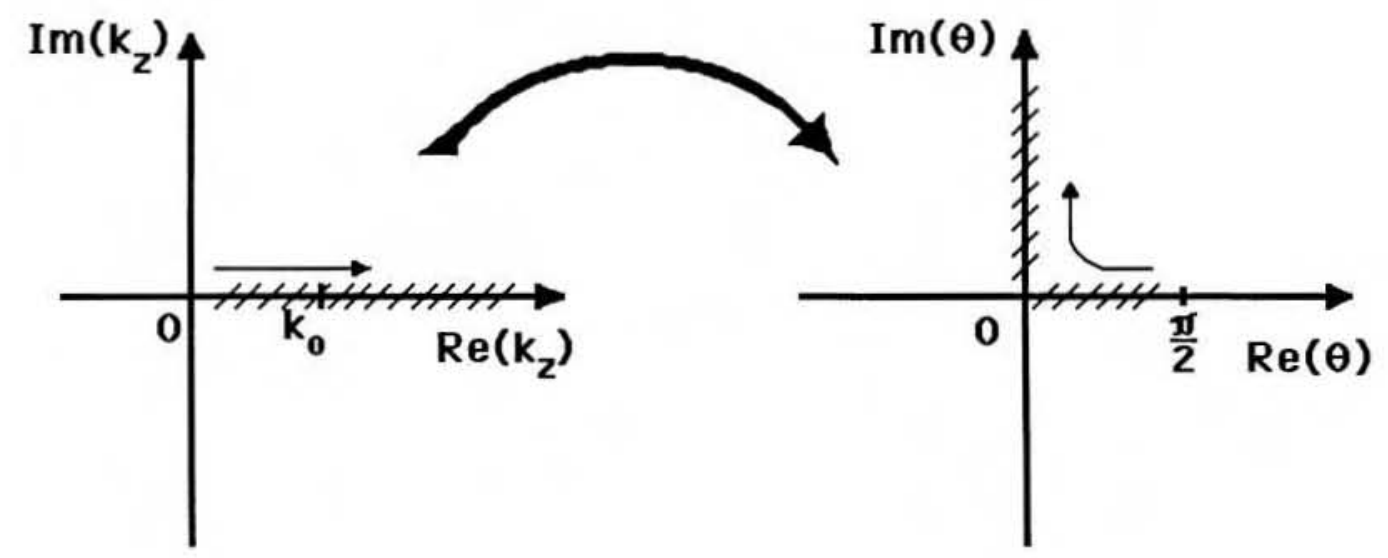

Fig. 6 Mapping from the $k_{z}-$ Plane into the $\theta$-Plane 


\subsection{Asymptotic Behavior of the Plane Wave Reflection Coefficient}

The input to both the Gelfand-Levitan algorithm and to the Born approximation is the Fourier transform of the reflection coefficient

$$
R(z)=\int_{-\infty}^{\infty} r\left(k_{z}\right) e^{i k z z} d k_{z}
$$

The properties of $r\left(\mathrm{k}_{\mathrm{z}}\right)$ as $\mathrm{k}_{\mathrm{z}} \rightarrow \infty$ are studied for two simple cases and then generalized.

(a) Half space

The reflection coefficient is given by

$$
r\left(k_{z}\right)=\frac{z_{1}-z}{z_{1}+z}
$$

where the impedance $\mathrm{z}=\rho \mathrm{c} / \cos \theta$ or

$$
\mathrm{z}=\frac{\rho \omega_{0}}{\mathrm{k}_{\mathrm{z}}}
$$

Therefore,

$$
r\left(k_{z}\right)=\frac{k_{z}-k_{z l}}{k_{z}+k_{z l}}
$$


And since $k_{x}$ is continuous,

$$
\mathrm{k}_{\mathrm{z} 1}^{2}=\left(\mathrm{k}_{1}^{2}-\mathrm{k}_{0}^{2}\right)+\mathrm{k}_{\mathrm{z}}^{2}
$$

and

$$
r\left(k_{z}\right)=\frac{k_{z}-\sqrt{\left(k_{l}^{2}-k_{0}^{2}\right)+k_{z}^{2}}}{k_{z}+\sqrt{\left(k_{l}^{2}-k_{0}^{2}\right)+k_{z}^{2}}}
$$

As $k_{z} \rightarrow \infty$

$$
\begin{aligned}
r\left(k_{z}\right) \sim & \frac{k_{z}-k_{z}\left(1+\frac{1}{2 k_{z}^{2}}\left(k_{1}^{2}-k_{0}^{2}\right)\right)}{k_{z}+k_{z}\left(1+\frac{1}{2 k_{z}^{2}}\left(k_{1}^{2}-k_{0}^{2}\right)\right.} \\
& =-\frac{1}{4}\left(k_{1}^{2}-k_{0}^{2}\right) / k_{z}^{2}
\end{aligned}
$$

Hence,

$$
r\left(k_{z}\right) \underset{k_{z} \rightarrow \infty}{\stackrel{k^{\prime}}{\longrightarrow}} 0 \text { as }\left(1 / \mathrm{k}_{\mathrm{z}}{ }^{2}\right)
$$

When density variations are considered,

$$
r\left(k_{z}\right)=\frac{m \cos \theta-n \cos \theta_{1}}{m \cos \theta+n \cos \theta_{1}}
$$


where $m=\rho_{1} / \rho$ and $n=k_{1} / k_{0}$

$$
r\left(k_{z}\right)=\frac{m k_{z}-k_{z l}}{m k_{z}+k_{z l}}
$$

As $\mathrm{k}_{\mathrm{z}} \rightarrow \infty$

$$
r\left(k_{z}\right) \sim\left(\frac{m-1}{m+1}\right)+\left(k_{1}{ }^{2}-k_{0}{ }^{2}\right) / 2 k_{z}{ }^{2}
$$

which still decays as $\left(1 / \mathrm{k}_{\mathrm{z}}{ }^{2}\right)$, but tends to a finite limit $\left(\frac{m-1}{m+1}\right)$ as $k_{z} \rightarrow \infty$

Therefore, the Fourier transform of $r\left(k_{z}\right)$ involves generalized functions,

$$
F\left(r\left(k_{z}\right)\right)=\left(\frac{m-1}{m+1}\right) \delta(z)+(\text { analytic function })
$$

(b) One Layer Case (cf. Figure 7).

The reflection coefficient at the $(0-1)$ interface is

$$
r\left(k_{z}\right)=\frac{\left(\frac{z_{1}-z_{0}}{z_{1}+z_{0}}\right)+\left(\frac{z_{2}-z_{1}}{z_{2}+z_{1}}\right) e^{2 i k_{1 z} d}}{1+\left(\frac{z_{1}-z_{0}}{z_{1}+z_{0}}\right)\left(\frac{z_{2}-z_{1}}{z_{2}+z_{1}}\right) e^{2 i k_{1 z}} d}
$$




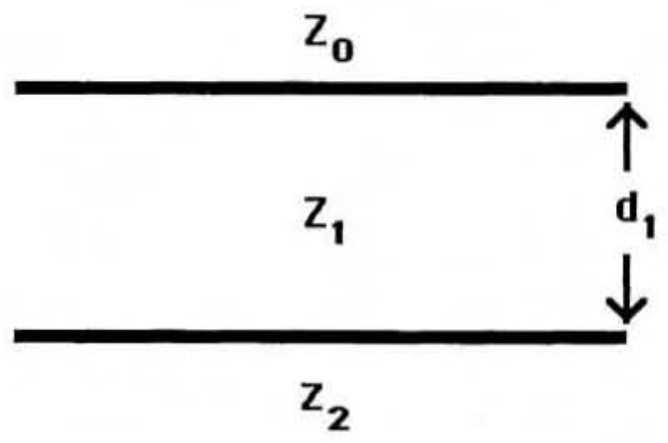

Fig. 7 One Layer Case 
The analysis of the terms within parentheses is identical to the analysis carried out in the previous section. One can conclude immediately that

$$
\begin{gathered}
r\left(\mathrm{k}_{\mathrm{z}}\right) \underset{\mathrm{k}_{\mathrm{z}} \rightarrow \infty}{\longrightarrow}\left[\left(\mathrm{k}_{1}{ }^{2}-\mathrm{k}_{0}{ }^{2}\right)+\left(\mathrm{k}_{2}{ }^{2}-\mathrm{k}_{1}{ }^{2}\right) \mathrm{e}^{2 \mathrm{ik} \mathrm{z}^{\mathrm{d}}}\right] / 4 \mathrm{k}_{\mathrm{z}}{ }^{2} \\
\longrightarrow 0 \text { as }\left(1 / \mathrm{k}_{\mathrm{z}}{ }^{2}\right)
\end{gathered}
$$

Note:

The delta function potential

$$
\mathrm{V}(\mathrm{z})=\mathrm{A} \delta(\mathrm{z})
$$

can be considered as a limiting case of the one-layer problem. It has associated with it a reflection coefficient ${ }^{(20)}$

$$
r\left(k_{z}\right)=-i \frac{A}{2 k_{z}+i A}
$$

which goes to zero as $k_{z}+\infty$, but only as $\left(1 / k_{z}\right)$ in apparent violation of the result just derived. The reason is that in a delta function potential, $\mathrm{V}(\mathrm{z}) \rightarrow \infty$ which implies $\left(\mathrm{k}_{0}{ }^{2}-\mathrm{k}_{1}{ }^{2}\right) \rightarrow \infty$, invalidating the binomial approximation to the square root used in section (a). However, the formulation of the acoustic problem requires $v(z) \leq k_{0}{ }^{2}$ which is finite ( as long as the frequency $\omega_{0}$ is finite). Therefore, 
delta-like potentials do not arise and the binomial approximation is valid.

(c) General Case

The expression for $r\left(k_{z}\right)$ given in the previous section can be rewritten in the general form

$$
\begin{aligned}
& r\left(k_{z}\right)=\frac{R_{01}+R_{12} e^{2 i k_{1 z} d_{1}}}{1+R_{01} R_{12} e^{2 i k_{1 z} d_{1}}} \\
& =\frac{1}{R_{01}}+\frac{\left[1-\left(1 / R_{01}\right)^{2}\right] e^{-2 i k_{1 z} d_{1}}}{\left(\frac{1}{R_{01}}\right) e^{-2 i k_{1 z} d_{1}}+R_{12}}
\end{aligned}
$$

The reflection coefficient can be readily generalized to include stratified media ( 58 )

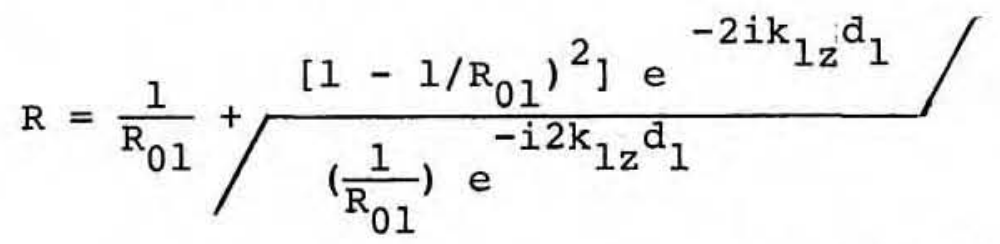

$$
\begin{aligned}
& +\frac{1}{R_{12}}+\frac{\left[1-\left(1 / R_{12}{ }^{2}\right)\right] e^{-i 2 k_{2 z}\left(d_{2}-d_{1}\right)}}{\left(\frac{1}{R_{12}}\right) e^{-i 2 k_{2 z}\left(d_{2}-d_{1}\right)}}+\ldots \\
& +\frac{1}{R_{(n-1) n}}+\frac{\left[1-\left(1 / R^{2}(n-1) n\right)\right] e^{-i 2 k} n z\left(d_{n}-d_{n-1}\right)}{\left[1 / R_{n-1) n-}\right] e^{-i 2 k_{n z}\left(d_{n}-d_{n-1}\right)}} \\
& +R_{n+1}
\end{aligned}
$$


The continued fraction representation of (equation 3.25) indicates that the partial reflection coefficient $R_{12}\left(k_{z}\right)$ in (equation 5.23) could represent the reflection coefficient due to a complicated medium rather than to a simple homogeneous half space.

The asymptotic behavior of $r\left(k_{z}\right)$ is deduced in a two step process. Assume the configuration of Figure 8 with $R_{1}\left(k_{z}\right) \rightarrow\left(\frac{\alpha}{k_{z}^{2}}\right)$ as $k_{z} \rightarrow \infty$ Adding a new interface to the set-up (Figure 9) and using equation (5.24),

$$
\begin{aligned}
r\left(k_{z}\right) & +\frac{k_{z}^{2}}{\beta}+\frac{\left(1-k_{z}^{4} / \beta^{2}\right) e^{-i 2 k z^{d}}}{\frac{k_{z}{ }^{2}}{\beta} e^{-2 i k z^{d} i}+\frac{\alpha}{k_{z}{ }^{2}}} \\
+\frac{k_{z}^{2}}{\beta}+\left(\frac{\beta}{k_{z}{ }^{2}}-\frac{k_{z}{ }^{2}}{\beta}\right)\left(1-\frac{\beta \alpha}{k_{z}{ }^{2}} e^{2 i k_{z} d_{1}}\right. & \left.+\frac{\beta^{2} \alpha^{2}}{k_{z}{ }^{2}} e^{4 i k_{z} d_{1}}+\ldots\right)
\end{aligned}
$$

Therefore,

$$
r\left(k_{z}\right) \underset{\mathrm{z}^{+\infty}}{\longrightarrow} 0 \text { as }\left(1 / \mathrm{k}_{\mathrm{z}}{ }^{2}\right)
$$




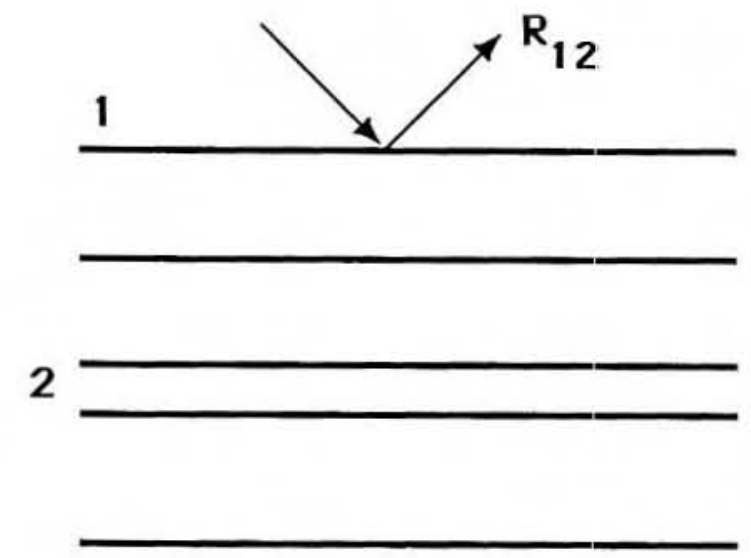

Fig. 8 Stack of Isovelocity Layers 


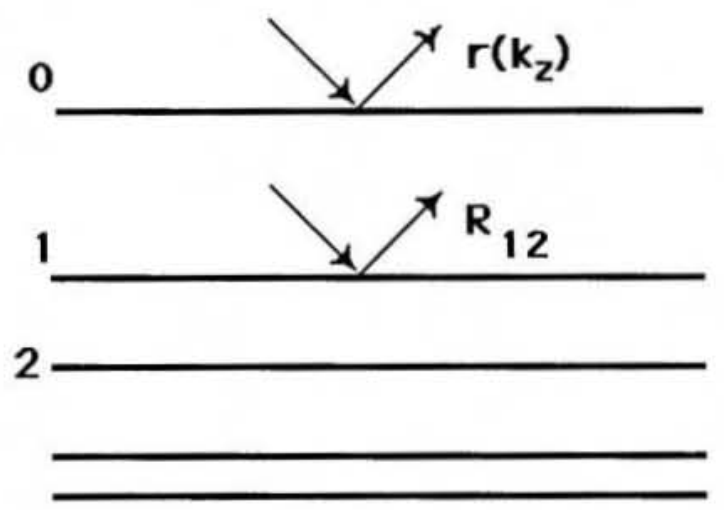

Fig.9 Adding a Top Layer to the Stack in Fig.8 
The argument just presented is, in fact, the last step in a proof by induction of the proposition that $r\left(k_{z}\right) \rightarrow 0$ as $\left(1 / \mathrm{k}_{\mathrm{z}}{ }^{2}\right)$ in the presence of a homogeneous half space. The proposition was proved true for one or two interfaces was assumed true for an arbitrary number of interfaces and was shown to hold for one more interface. This result should be contrasted with the corresponding situation in the Ware and Aki experiment; For angles of incidence greater than critical, they were confronted with the fact that $r(\omega) \rightarrow 1$, as $\omega \rightarrow \infty$, and could not proceed with the Gelfand-Levitan inversion procedure. 
This chapter presents the derivation of our approach to the one-dimensional inverse problem. The results yield the methodology underlying the numerical computations described in Chapter $V$. The derivation exploits the equivalence between the acoustic problem and the corresponding quantum scattering problem as presented in Chapter II. A major difference between the two is the boundary conditions. Whereas in quantum mechanics, the unknown slab is surrounded by isovelocity space (zero end potential), in the acoustics problem, as applied to the ocean bottom, differing velocities have to be accommodated above and below the slab (non-zero end potential). The solution to the inverse problem detailed in this chapter consists of an extension of Faddeev's method(16) for deriving the scattering matrix in the case of zero end potential (or integrable potential) to the case of non-zero end potential which is representative of the reflections from the bottom of the ocean. We are able to solve the problem analytically, in part, because of its one-dimensional modeling. Our technique is related to that applied by stickler to a similar scattering problem. 


\subsection{Introduction}

The determination of the potential $V(z)$ in the Schrodinger equation

$$
\frac{d^{2} \psi}{d z^{2}}+\left[p^{2}-v(z)\right] \psi(z)=0
$$

from scattering data such as the reflection coefficient constitutes the one-dimensional inverse problem.

Two scattering solutions of the Schrodinger equation are defined by their asymptotic behavior ( $p$ is the vertical wavenumber also referred to as $\mathrm{k}_{\mathrm{z}}$ ):

$$
\begin{aligned}
& \psi_{1}(z, p)= \begin{cases}e^{i p z}+s_{12}(p) e^{-i p z} \text { as } & z+-\infty \\
s_{11}(p) e^{i p z} & z+\infty\end{cases} \\
& \psi_{2}(z, p)=\left\{\begin{array}{ll}
e^{-i p z}+s_{21}(p) e^{i p z} & \text { as } \\
s_{22}(p) e^{-i p z} & z++\infty
\end{array} .\right.
\end{aligned}
$$

The matrix of coefficients

$$
\left(\begin{array}{ll}
s_{11}(p) & s_{12}(p) \\
s_{21}(p) & s_{22}(p)
\end{array}\right)
$$


is known as the $\mathrm{S}$ matrix of the Schrodinger equation. The element $s_{12}(p)$ corresponds to the plane wave reflection coefficient determined in the WHOI experiment.

Faddeev $(16 \mathrm{~B})$ has shown that $\mathrm{s}_{12}(\mathrm{p})$ may determine all the elements of the S matrix and hence is sufficient to obtain the scattering potential $V(z)$. However, these results have an important practical restriction, namely, that the end potential tends to zero; i.e., $V(z) \rightarrow 0$ as $z+ \pm^{\infty}$. They are reviewed in the first part of this chapter.

In the second part of the chapter, we present an extension of the theory to include the geophysically significant case of a finite end potential, $\mathrm{V}(\mathrm{z})+\mathrm{V}_{1}$ as $\mathrm{z} \rightarrow \infty$. Finally, an appropriate choice of source frequency is shown to eliminate trapped modes.

\subsection{Properties of the Solution of the Schrödinger}

Equation $\left(\mathrm{V}_{1}=0\right)$

Two fundamental solutions of the Schrodinger equation are introduced,

$$
\begin{aligned}
& \mathrm{u}_{1}(\mathrm{z}, \mathrm{p}) \sim \mathrm{e}^{i p z} \text { as } \mathrm{z}++\infty \\
& \mathrm{u}_{2}(\mathrm{z}, \mathrm{p}) \sim \mathrm{e}^{-i p \mathrm{p}} \text { as } \mathrm{z} \rightarrow-\infty
\end{aligned}
$$


The method of variation of parameters leads to a representation of $u_{1}(z, k)$ and $u_{2}(z, k)$ as solutions of Volterra equations of the second kind(16)

$$
\begin{aligned}
& u_{1}(z, p)=e^{i p z}-\int_{z}^{\infty} \frac{\operatorname{sinp}\left(z-z^{\prime}\right)}{p} v(z) u_{1}\left(z^{\prime}, p\right) d z^{\prime} \\
& u_{2}(z, p)=e^{-i p z}+\int_{-\infty}^{z} \frac{\operatorname{sinp}\left(z-z^{\prime}\right)}{p} v(z) u_{2}\left(z^{\prime}, p\right) d z^{\prime} .
\end{aligned}
$$

But, since the Schrodinger equation is symmetrical in $p$, $u_{1}(z,-p)$ and $u_{2}(z,-p)$ are also solutions of (eq. 4.1). The solution pairs $\left[u_{1}(z, p), u_{1}(z,-p)\right]$ and $\left[u_{2}(z, p), u_{2}(z,-p)\right]$ are linearly independent since their Wronskians obtained from their asymptotic form (eqs. $4.4,4.5$ )

$$
\begin{aligned}
& \mathrm{w}\left(\mathrm{u}_{1}, \mathrm{u}_{1}{ }^{*}\right)=2 \mathrm{ip} \\
& \mathrm{w}\left(\mathrm{u}_{2}, \mathrm{u}_{2}{ }^{*}\right)=-2 i p
\end{aligned}
$$

are non-zero for $p \neq 0$.

Now, any solution of the Schrödinger equation can be written as a linear combination of two independent solutions. In particular, 


$$
\begin{aligned}
& u_{1}(z, p)=u_{2}(z, p) c_{22}(p)+u_{2}(z,-p) c_{21}(p) \\
& u_{2}(z, p)=u_{1}(z, p) c_{11}(p)+u_{1}(z,-p) c_{12}(p) .
\end{aligned}
$$

The next section examines some properties of the coefficients $c_{i j}(k)$ which will later be shown to be closely related to the elements of the $\mathrm{S}$ matrix.

\subsubsection{Properties of the Coefficients $c_{j j}(p)$}

The coefficients $c_{i j}(p)$ can be expressed as Wronskians by "taking Wronskians" of both sides in equations ( 4.9 and 4.10). For instance, from (equation 4.9)

$$
\begin{aligned}
W\left(u_{1}(z, p), u_{2}(z, p)\right)= & w\left[u_{1}, u_{1}\right] c_{11}(p) \\
& +W\left[u_{1}(z, p), u_{1}(z,-p)\right] c_{12}(p) .
\end{aligned}
$$

We know, however, that in (4.11)

$$
\mathrm{W}\left[\mathrm{u}_{1}, \mathrm{u}_{1}\right]=0 \quad \text { (linearly dependent functions) }
$$


and

$$
\mathrm{w}\left[\mathrm{u}_{1}(\mathrm{z}, \mathrm{p}), \mathrm{u}_{1}(\mathrm{z},-\mathrm{p})\right]=2 \mathrm{ip} \quad(\mathrm{cf}, \text { eq. } 4.8) \text {. }
$$

It follows that

$$
c_{12}(p)=\frac{1}{2 i p} w\left[u_{1}(z, p), u_{2}(z, p)\right]
$$

One can show similarly that

$$
\begin{aligned}
& c_{21}(p)=c_{12}(p) \\
& c_{11}(p)=-c_{22}(-p)=\frac{1}{2 i k} w\left[u_{2}(z,-p), u_{1}(z, p)\right]
\end{aligned}
$$

Using the values of $c_{i j}$ expressed in this form, and substituting eq. 4.9 into eq. 4.10 , one gets the compatibility relations,

$$
\begin{aligned}
& c_{11}(p) c_{22}(p)+c_{21}(p) c_{12}(-p)=1 \\
& \left|c_{12}(p)\right|^{2}-\left|c_{11}(p)\right|^{2}=1 .
\end{aligned}
$$


and,

$$
c_{12}(p) c_{22}(p)+c_{11}(-p) c_{21}(p)=0 .
$$

The asymptotic form of $u_{2}(z, k)$ in (eq. 4.7) for $z+\infty$,

$$
\begin{aligned}
u_{2}(z, p)= & e^{-i p z}\left[1-\frac{1}{2 i p} \int_{-\infty}^{\infty} e^{i p z^{\prime}} v\left(z^{\prime}\right) u_{2}\left(z^{\prime}\right) d z^{\prime}\right] \\
& +\frac{e^{i p z}}{2 i p} \int_{-\infty}^{\infty} e^{-i p z^{\prime}} v\left(z^{\prime}\right) u_{2}\left(z^{\prime}\right) d z^{\prime}
\end{aligned}
$$

leads by comparison with (eq. 4.9) to the identifications:

$$
\begin{aligned}
& c_{12}(p)=1-\frac{1}{2 i p} \int_{-\infty}^{\infty} e^{i p z^{\prime}} v\left(z^{\prime}\right) u_{2}\left(z^{\prime}\right) d z^{\prime} \\
& c_{11}(p)=\frac{1}{2 i n} \int_{-\infty}^{\infty} e^{-i p z^{\prime}} v\left(z^{\prime}\right) u_{2}\left(z^{\prime}\right) d z^{\prime} .
\end{aligned}
$$


Using the previous estimates for $\mathrm{u}_{2}$ in eq. 4.5 and the Riemann-Lebesque theorem, it can be seen, that for large $p$, $c_{12}(p)-1$ is $o(1 / p)$ and $c_{11}(p)$ is o $\left(\frac{1}{p}\right) \cdot{ }^{*}$ Moreover, $c_{12}(p)$ is analytic in the upper half plane, does not vanish on the real $p$ axis, and has only a finite number of simple zeros on the imaginary axis.

\subsubsection{Properties of the $s_{i j}(k)$ Coefficients}

One is now ready to return to the original scattering problem and its associated $\mathrm{S}$ matrix.

$$
\psi_{1}(z, p) \text {, the solution of the scattering problem of }
$$

interest, can be written in terms of the linearly independent solutions $\mathrm{u}_{2}(\mathrm{z}, \mathrm{p})$ and $\mathrm{u}_{2}(\mathrm{z},-\mathrm{p})$

$$
\begin{aligned}
\psi_{1}(z, p) & =u_{2}(z,-p)+s_{12}(p) u_{2}(z, p) \\
& =s_{11}(p) u_{1}(z, p) .
\end{aligned}
$$

The order symbol o( ) is defined as follows:

$$
\begin{aligned}
& \mathrm{f}(\varepsilon)=\mathrm{o}[\mathrm{g}(\varepsilon)] \text { as } \varepsilon \rightarrow 0 \\
& \text { if } \lim _{\varepsilon \rightarrow 0} \frac{\mathrm{f}(\varepsilon)}{\mathrm{g}(\varepsilon)}=0
\end{aligned}
$$


Writing out $u_{1}(z, p)$ in terms of $u_{2}(z, \pm p)$ as given in eq. 4.10 , one obtains the compatibility relations

$$
\begin{aligned}
& s_{11}(p)=\frac{1}{c_{21}(p)} \\
& s_{12}(p)=\frac{c_{22}(p)}{c_{21}(p)} .
\end{aligned}
$$

Carrying out the same operation on $\psi_{2}(z, k)$, one obtains

$$
\begin{aligned}
& s_{22}(p)=\frac{1}{c_{12}(p)} \\
& s_{21}(p)=\frac{c_{11}(p)}{c_{12}(p)} .
\end{aligned}
$$

From the asymptotic behavior of the Jost functions $c_{i j}$ one can deduce the asymptotics of the $S$ matrix*

$$
\begin{aligned}
& s_{11}(p)=s_{22}(p)=1+o(1 / p) \\
& s_{12}(p) \text { and } s_{21}(p)=O(1 / p)
\end{aligned}
$$

The order symbol $O($ ) is defined as follows:

$$
\begin{aligned}
& f(\varepsilon)=O[g(\varepsilon)] \text { as } \varepsilon \rightarrow 0 \\
& \text { if } \lim _{\varepsilon+0} \frac{f(\varepsilon)}{g(\varepsilon)}=A, 0<|A|<\infty
\end{aligned}
$$


It can be seen from eq. 4.24 , eq. 4.16 and eq. 4.17 that the $\mathrm{S}$ matrix is unitary,

$$
\left|s_{11}\right|^{2}+\left|s_{12}\right|^{2}=\left|s_{22}\right|^{2}+\left|s_{21}\right|^{2}=1 \text {, }
$$

implying conservation of energy, and that since $s_{i j}(-p)=$ $s^{*}{ }_{i j}(p)$,

$$
\mathrm{s}_{11} \mathrm{~s}_{21}+\mathrm{s}_{12} \mathrm{~s}_{22}=0
$$

The coefficients $s_{i j}(p)$ are continuous for real

$p, s_{11}(p)$ being analytic in the upper half plane except for poles on the imaginary axis (corresponding to the zeros of $\left.c_{12}(p)\right)$. Conditions $(4.26)$ and (4.27) allow one to reconstruct the scattering matrix from a knowledge of the reflection coefficient $s_{12}(p)$. In what follows, $s_{12}(p)$ is identified with the plane wave reflection coefficient $r\left(k_{z}\right)$ and $s_{11}(p)$ with the transmission coefficient $t\left(k_{z}\right)$. Substituting $c_{21}$ by $(1 / t)$ and $c_{22}$ by $(r / t)$ in $(e q .4 .10)$, it follows that

$$
t(p) u_{1}(z, p)=r(p) u_{2}(z, p)+u_{2}(z,-p) \text {. }
$$

The above equation is the basis for the derivation of the Gelfand-Levitan inversion method that has been obtained for a potential $V(z)+0$ as $z+ \pm^{\infty}$. Such a potential arises 
in the study of dielectric slabs by electromagnetic probing. In the earth, however, the velocity $c(z)$ tends to a value $c_{1}$ larger than the surface velocity $c_{0}$. Correspondingly, the potential $\mathrm{V}(\mathrm{z})$ tends to a positive constant $\mathrm{V}_{1}$,

$$
\begin{aligned}
\mathrm{v}_{1} & =\mathrm{k}_{0}^{2}\left(1-\frac{\mathrm{c}_{0}{ }^{2}}{\mathrm{c}_{1}{ }^{2}}\right) \\
& =\mathrm{k}_{0}{ }^{2}-\mathrm{k}_{1}{ }^{2} .
\end{aligned}
$$

Therefore the Gelfand-Levitan inversion does not really apply, and a scattering solution is needed that allows a nonzero end potential. In the next section, we present our approach to this problem.

\subsection{Non Zero Final Potential}

To accommodate to a non-zero $\mathrm{V}_{1}$, we now consider the fundamental solution of the Schrodinger equation $u_{1}(z, p)$ defined asymptotically as

$$
u_{1}(z, p) \sim e^{i p^{\prime} z} \quad z+\infty
$$

where

$$
p^{\prime}=\sqrt{p^{2}-v_{1}} \text {. }
$$


We assume Imp'>0, in order to satisfy the radiation condition at $\infty$. The Volterra integral equation representation of $u_{1}(z, p)$ in eq. 4.6 is modified to

$$
u_{1}(z, p)=e^{i p^{\prime} z}-\int_{z}^{\infty} \frac{\sin p^{\prime}\left(z-z^{\prime}\right)}{p^{\prime}}\left[v(z)-v_{1}\right] u_{1}\left(z^{\prime}, p\right) d z^{\prime}
$$

while the integral equation representation of $u_{2}(z, p)$ is unchanged

$$
u_{2}(z, p)=e^{-i p z}+\int_{-\infty}^{z} \frac{\sin p\left(z-z^{\prime}\right)}{p} v(z) u_{2}\left(z^{\prime}, p\right) d z^{\prime}
$$

\subsubsection{Asymptotic Behavior}

The key observations to be made relative to eq. 4.31 is that since $u_{1}(z, p)$ is the solution of a Volterra equation of the second kind with square integrable kernel, the method of successive approximation will converge. That observation has in fact been applied by $D$. Stickler 7 in connection with the Deift-Trubowitz inversion procedure; thus, we have

$$
u_{1}(z, p)=\left[1-\frac{v(z)-V_{1}}{(2 i p)^{2}}\right] e^{\left.i p z-\frac{i v_{1}}{2 p} z-\frac{1}{2 i p_{z}^{\infty}} \int_{(I m p>0)}^{\infty}\left(z^{\prime}\right)-v_{1}\right) d z^{\prime}}+H \cdot O \cdot T .^{*}
$$

H.O.T. stands for higher order terms 
from which the transmission coefficient $t(p)$ can be deduced

$$
t(p)=\left(1-\frac{v_{1}}{(2 i p)^{2}}\right) e^{i \frac{v_{1} z_{0}}{2 p}+\frac{1}{2 i p} \int_{-\infty}^{z_{0} 0} V^{\prime}\left(z^{\prime}\right) d z^{\prime}+\frac{1}{2 i p} \int_{z_{0}}^{\infty}\left(V\left(z^{\prime}\right)-v_{1}\right) d z^{\prime}}+\text { +H.о.T. }
$$

where $z_{0}$ is arbitrary.

Similarly, (Imp >0)

$$
u_{2}(z, p)=\left(1-\frac{V(z)}{(2 i p)^{2}}\right) e^{-i p z-\frac{1}{2 i p}} \int_{-\infty}^{z} \begin{array}{r}
v^{\prime}\left(z^{\prime}\right) d z^{\prime} \\
+ \text { H.о.T. }
\end{array}
$$

\subsubsection{Inversion Procedure}

We are now ready to present the inversion procedure for $\mathrm{v}_{1}>0$. Note that the basic relation (eq. 4.28) still holds for $v_{1}>0$,

$$
t(p) u_{1}(z, p)=r(p) u_{2}(z, p)+u_{2}(z,-p)
$$

Following Faddeev's(16) case of $\mathrm{V}=0$, for the case of $\mathrm{v}_{1}>0$, a function $\mathrm{h}(\mathrm{z}, \mathrm{p})=\mathrm{u}_{2}(\mathrm{z}, \mathrm{p}) \mathrm{e}^{\mathrm{ipz}}$ is introduced. The expression for $u_{2}(z, p)$ (eq. 4.35) shows that $h(z, p)-1$ is analytic in the upper half plane and $\operatorname{Im}(p)>0$ and $\rightarrow 0$ as $|p|+\infty$. We thus obtain $(\varepsilon \rightarrow 0+)$ 


$$
h(z, p)-1=\frac{1}{2 \pi i} \int_{-\infty}^{\infty} \frac{h\left(z, p^{\prime}\right)-1}{p^{\prime}-p-i \varepsilon},
$$

or usind (eq. 4.36 )

$$
\begin{aligned}
h(z, p)-1= & \frac{1}{2 \pi i} \int_{-\infty}^{\infty} d p^{\prime} \frac{t\left(p^{\prime}\right) u_{1}\left(z, p^{\prime}\right) e^{-i p^{\prime} z}-1}{p^{\prime}+p+i \varepsilon} \\
& -\frac{1}{2 \pi i} \int_{-\infty}^{\infty} \frac{r\left(p^{\prime}\right) u_{2}\left(z, p^{\prime}\right) e^{-i p^{\prime} z}}{p^{\prime}+p+i \varepsilon} d p^{\prime}
\end{aligned}
$$

The first inteqral in (eq. 4.38) is zero since

$$
t(p) u_{1}(z, p) e^{-i p z}-1=-\frac{v(z)}{(2 i p)^{2}} e^{\frac{1}{2 i p} \int_{-\infty}^{z} v\left(z^{\prime}\right) d z^{\prime}}+\text { H.O.T. }
$$

thus

$$
h(z, p)-1=-\frac{1}{2 \pi i} \int_{-\infty}^{\infty} \frac{r\left(p^{\prime}\right) u_{2}\left(z, p^{\prime}\right) e^{-i p^{\prime} z}}{p^{\prime}+p+i \varepsilon} d p^{\prime} .
$$

Comparing eqs. (4.37) and $(4.38 a)$, we can express $u_{2}\left(z, p^{\prime}\right)$ in the Levin representation (17),

$$
u_{2}(z, p)=e^{-i p z}+\int_{-\infty}^{z} K\left(z, z^{\prime}\right) e^{i p z^{\prime}} d z^{\prime}
$$

in which the kernel of the integral does not depend on $p$; i.e., 


$$
K(z, y)=-\frac{1}{2 \pi} \int_{-\infty}^{\infty} r(p) u_{2}(z, p) e^{-i p y} d p .
$$

Inserting (eq. 4.40) into (eq. 4.41) results in the GelfandLevitan type equation

$$
\begin{aligned}
K(z, y)+R(z+y)+\int_{-\infty}^{z} R\left(z^{\prime}+y\right) K\left(z, z^{\prime}\right) d z^{\prime} & =0 \\
y< & z
\end{aligned}
$$

where $R(z)$ is the Fourier transform of $r(p)$

$$
R(z)=\frac{1}{2 \pi} \int_{-\infty}^{\infty} r(p) e^{-i p z} d p
$$

When (eq. 4.41) is substituted into the Schrodinger equation, it is found that $K(z, y)$ satisfies a partial differential equation

$$
\frac{\partial^{2} K}{\partial z^{2}}-\frac{\partial^{2} K^{2}}{\partial y^{2}}-V(z) K=0
$$

subject to the boundary conditions

$$
\begin{aligned}
& K(z,-\infty)=0 \\
& V(z)=2 \frac{d K}{d z}(z, z) .
\end{aligned}
$$


The solution of $R(z)$ in eq. 4.43 consists, in general, of both a continuous and a discrete part. The discrete part applies in the presence of trapped modes. In that case, additional information is required to construct $R(z)$

$$
R(z)=\frac{1}{2 \pi} \int_{-\infty}^{\infty} r(p) e^{-i p z} d p+\sum_{i} m_{i} e^{-i p i^{z}} .
$$

where the $p_{i}$ 's are the poles of $r(p)$ on the positive imaginary axis.

The choice of the constant normalization coefficients $m_{i}$ is dictated by the requirement that $V(z)=0$ for $z<0$. This can be seen by examining the Gelfand-Levitan equation (4.42),

$$
\begin{aligned}
K(z, y)+R(z+y)+\int_{-\infty}^{z} R\left(z^{\prime}+y\right) k\left(z, z^{\prime}\right) d z^{\prime} & =0 \\
y & <z
\end{aligned}
$$

We note that $R(z)=0$ for $z<0$ insures that $k(z, y)$, and hence that $V(z)=2 \frac{d K}{d z}(z, z)$ are all zero for $z<0$. The choice of $\mathrm{m}_{\mathrm{i}}$ in (eq. 4.47) is therefore dictated by the requirement $R(z)=0$ for $z<0$. Now, the integral in (eq. 4.47 ) is for $z<0$,

$$
\frac{1}{2 \pi} \int_{-\infty}^{\infty} r(p) e^{-i p z} d p=i \sum_{i} b_{i} e^{-p_{i} z}
$$

where the $b_{i}$ 's are the residues at the poles $p_{i}$ of $r(p)$. Substituting for the value of the integral in (eq. 4.47), and imposing $R(z)=0$ for $z<0$ yields $m_{i}=-i b_{i}$. 
In principle, in order to solve for $R(z)$, an additional measurement would be required to obtain the residues $b_{i}$. However, the next section demonstrates that, in the presence of slow velocity layers in the sediment, the frequency can be selected low enough to eliminate the trapped modes (or bound states of the Schrodinger equation), and thus the continuous part of the solution for $R(z)$ will suffice.

\subsection{Bound States}

The potential diagram (Fig. 3) indicates that bound states may occur due to the presence of a low velocity zone near the water sediment interface. Bound states are square integrable solutions of Schrödinger's equation and, as will be shown later, present considerable difficulty in the inversion procedure. The number of bound states $M$ was obtained by Bargmann (59)

$$
M \leqslant \int_{0}^{\infty} z\left|V_{-}(z)\right| d z \leqslant M+I
$$

where $V_{-}(z)$ is the negative portion of the potential for $z>0$. It is clear that the number of bound states is determined by the width of the low velocity zone (prescribed by the deology) and by the depth of the potential well which is a function of the frequency at which the experiment is conducted. 
To eliminate bound states, we impose the condition:

$$
\int_{0}^{\infty} z\left|v_{-}(z)\right| d z<1 .
$$

Now,

$$
\int_{0}^{\infty} z\left|v_{-}(z)\right| d z<\frac{1}{2} \ell^{2}\left|v_{-}(z)\right|_{\max }
$$

where $\ell$ is the width of the well and $\left|v_{-}(z)\right|_{\max }$ its maximum depth:

$$
\left|v_{-}(z)\right|_{\max }=\left|\frac{\omega^{2}}{c_{0}^{2}}\left(1-\frac{c_{0}^{2}}{c_{\min }^{2}}\right)\right|
$$

The condition expressed by eq. (4.12) is satisfied when

$$
\left(\frac{\ell \omega}{c_{0}}\right) \quad\left|1-\frac{c_{0}^{2}}{c_{\min }^{2}}\right|<\sqrt{2}
$$

In particular, for $\frac{\ell \omega}{c_{\min }}<\sqrt{2}$, there will be no trapped modes. This simplified condition is a refinement of Stickler's result that $\left(\frac{\ell \omega}{c_{0}}\right)$ should be sufficiently small $(4.50)$. We have found that either of the two simplified conditions is too restrictive in practice and one should use our full equation (4.54). Hamilton has studied the characteristics of surface sound channels in marine sediments $(60)$. The velocity ratio $\mathrm{R}=\left(\mathrm{c}_{\mathrm{min}} / \mathrm{c}_{0}\right)$ ranges from 0.984 for pelaqic clay to 0.99 for terrigeneous sediments, while the height of the channel depends on the velocity gradient a 


$$
\ell=c_{0}(1-R) / a \text {. }
$$

Therefore, for $\mathrm{R} \sim 1$, the condition of zero bound states (eq. $4.54)$ can be written

$$
\omega<a(1-R)^{-3 / 2} .
$$

A marked improvement in the bound obtains if a linear velocity profile

$$
c(z)=a z+c_{\min }
$$

with

$$
c(l)=c_{0}
$$

is assumed in the condition of zero bound states (eq. 4.51). The corresponding potential is then

$$
V(z)=-k_{0}^{2}\left(1-\frac{c_{0}^{2}}{\left(a z+c_{m i n}\right)^{2}}\right) \text { for } 0<z<\ell
$$

Substituting this $V(z)$ into eq. 4.51 and integrating by parts yields 


$$
-\frac{\omega^{2}}{a^{2}}\left[(1-R)+\frac{1}{2}(1-R)^{2}+\ell n R\right]<1 .
$$

For $\mathrm{R} \sim \mathrm{l}$,

$$
-\ln R \cong(1-R)+\frac{1}{2}(1-R)^{2}+\frac{1}{3}(1-R)^{3}
$$

Therefore,

$$
\omega<a \sqrt{3}(1-R)^{-3 / 2}
$$

Table I presents the upper bound on the probing frequency with the condition of zero bound states for representative values ${ }^{*}$ of $a$ and $R$ in the abyssal plain environment(60). The current frequency of operation(2), $220 \mathrm{~Hz}$, is low enough to eliminate the bound states in clayey silt and silty clay. It is assumed that for operations in clay sediments, an acoustic source will be available at about half the current frequency which would be sufficient to do away with the possible bound states.

The velocity gradient (a) can assume values over a wider range, than shown in Table I. For instance, Frisk et al., 4 have inferred from experimental data that (a) ranged for $0.5 s^{-1}$ to $2.9 s^{-1}$ at three locations in the Icelandic Basin. 
Table I: Upper bound on the probing frequency for the condition of zero bound states as a function of velocity ratio, $R$, and velocity qradient, a.

\begin{tabular}{|c|c|c|c|}
\hline $\mathrm{R}$ & Clayey Silt & Silty Clay & $\begin{array}{r}\text { Clay } \\
0.999\end{array}$ \\
\hline $1 \mathrm{~s}^{-1}$ & $8.7 \mathrm{kHz}$ & $275 \mathrm{~Hz}$ & $136 \mathrm{~Hz}$ \\
\hline $1.2 \mathrm{~s}^{-1}$ & $10.4 \mathrm{kHz}$ & $330 \mathrm{~Hz}$ & $162 \mathrm{~Hz}$ \\
\hline $1.3 \mathrm{~s}^{-1}$ & $11.3 \mathrm{kHz}$ & $358 \mathrm{kHz}$ & $176 \mathrm{~Hz}$ \\
\hline
\end{tabular}


CHAPTER V

\section{NUMERICAL SOLUTION OF THE GELFAND-LEVITAN EQUATION}

\subsection{Introduction}

The Gelfand-Levitan Equation

$$
R(z+y)+K(z, y)+\int_{-\infty}^{z} K\left(z, z^{\prime}\right) R\left(y^{\prime}+z^{\prime}\right) d z^{\prime}=0
$$

is a Fredholm equation of the second kind in the variable $y$ with $\mathrm{z}$ regarded as a parameter. The object of this chapter is to present three methods of computing $\mathrm{K}(\mathrm{z}, \mathrm{z})$ and hence to reconstruct the potential $\mathrm{V}(\mathrm{z})$.

\subsection{Series Expansion}

A parameter $\lambda$ is introduced in the Gelfand-Levitan equation

$$
R(z+y)+K(z, y)+\lambda \int_{-y}^{z} K\left(z, z^{\prime}\right) R\left(y+z^{\prime}\right) d z^{\prime}
$$

and a solution is sought by a method of successive approximations. The solution is written as a power series in $\lambda$

$$
K(z, y)=K_{0}(z, y)+\lambda K_{1}(z, y)+\lambda^{2} K_{2}(z, y)+\ldots
$$


Integrating term by term and equating coefficients of equal power of $\lambda$ one gets

$$
\begin{aligned}
& K_{0}(z, y)=-R(z+y) \\
& K_{1}(z, y)=-\int_{-y}^{z} R\left(y^{\prime}+z^{\prime}\right) K_{0}\left(z, z^{\prime}\right) d z^{\prime}
\end{aligned}
$$

and in general

$$
K_{n}(z, y)=-\int_{-y}^{z} R\left(y+z^{\prime}\right) K_{n-1}\left(z, z^{\prime}\right) d z^{\prime}
$$

The theory of Volterra integral equations of the second kind demonstrates that the series is convergent for all $\lambda$ when the norm of $R$,

$$
|| R||=\int_{-z}^{z} R^{2}(z+y) d y
$$

exists. ${ }^{(61)}$ But, Parseval's identity indicates that ||$R||$ is always finite since

$$
|| R|| \leqslant \int_{-\infty}^{\infty} R^{2}(y) d y=\frac{1}{2 \pi} \int_{-\infty}^{\infty}|b(p)|^{2} d p
$$

Hence, $b(p)$, given $|b(p)| \leqslant 1$ and $b(p) \underset{p \rightarrow \infty}{\longrightarrow}\left(1 / p^{2}\right)$, is square integrable. 
The $\mathrm{K}_{\mathrm{n}}(\mathrm{z}, \mathrm{y})$ are bounded by

$$
\left|K_{n}(z, y)\right|<|| R||^{2} \sqrt{\frac{A B(n-1)}{(n-1) !}}
$$

where $A=\int_{-y}^{z} R^{2}\left(y^{\prime}+z^{\prime}\right) d z^{\prime}$ and $B=\int_{-y}^{z} \int_{-y}^{z} d y^{\prime} d y^{\prime \prime} R^{2}\left(y^{\prime}+y^{\prime \prime}\right)$

\subsubsection{The Potential}

The expansion of $\mathrm{K}(\mathrm{z}, \mathrm{y})$ yields a corresponding expansion for the potential $V(z)=2 \frac{d K(z, z)}{d z}$,

$$
V(z)=V^{(0)}+V^{(1)}+v^{(2)}+\cdots
$$

where,

$$
\begin{aligned}
& V^{(0)}=2 \frac{d K_{0}}{d z}(z, z)=-2 \frac{d R}{d z}(2 z) \\
& V^{(1)}=2 \frac{d K_{1}}{d z}(z, z)=4 R^{2}(2 z)
\end{aligned}
$$

The computation of $\mathrm{V}^{(2)}$ is more involved and is presented here for reference purposes

$$
K_{2}(z, y)=\int_{-y}^{z} \int_{-z^{\prime}}^{z} R\left(y^{\prime} z^{\prime}\right) R\left(z^{\prime}+z^{\prime \prime}\right) R\left(z+z^{\prime \prime}\right) d z^{\prime \prime} d z^{\prime}
$$


which can be written

$$
K_{2}(z, y)=\int_{-z^{\prime}}^{z} d z^{\prime} \Phi\left(z, z^{\prime}\right)
$$

where

$$
\Phi\left(z, z^{\prime}\right)=\int_{-z^{\prime}}^{z} R\left(z^{\prime}+z^{\prime}\right) R\left(z^{\prime}+z^{\prime \prime}\right) R\left(z+z^{\prime \prime}\right) d z^{\prime \prime} \text { (5.15) }
$$

Therefore,

$$
\frac{d K_{2}}{d z}(z, z)=\Phi(z, z)+\phi(z,-z)+\int_{-z}^{z} d z^{\prime} \frac{\partial \Phi}{\partial z}\left(z, z^{\prime}\right)
$$

But,

$$
\Phi(z,-z)=0 .
$$

and

$$
\begin{aligned}
& \Phi(z, z)=R(2 z) \int_{0}^{2 z} R^{2}\left(z^{\prime}\right) d z^{\prime} \\
& \frac{\partial \Phi}{\partial z}\left(z, z^{\prime}\right)=R(2 z) R^{2}\left(z+z^{\prime}\right)+R\left(z+z^{\prime}\right) \int_{-z^{\prime}}^{z} \frac{\partial}{\partial z}\left(R\left(z+z^{\prime \prime}\right)\right) R^{\prime}\left(z^{\prime}+z^{\prime \prime}\right) d z^{\prime \prime}
\end{aligned}
$$


Finally,

$$
\begin{aligned}
& V^{(2)}(z)=4 R(2 z) \int_{0}^{2 z} R^{2}\left(z^{\prime}\right) d z^{\prime}+ \\
& +2 \int_{-z}^{z} \int_{-z^{\prime}}^{z} R\left(z^{\prime}+z^{\prime}\right) R\left(z^{\prime}+z^{\prime \prime}\right) \frac{\partial R}{\partial z}\left(z^{\prime}+z^{\prime}\right) d z^{\prime \prime} d z^{\prime}
\end{aligned}
$$

$\mathrm{V}^{(2)}(\mathrm{z})$ displays the global character of the higher order terms (which become increasingly unwieldy).

To summarize, the following approximation will be used

$$
\hat{V}(z)=-2 \frac{d R}{d z}(2 z)+4 R^{2}(2 z)
$$

\subsubsection{Connection with Other Formulations}

To first order,

$$
\hat{\mathrm{V}}(\mathrm{z})=-2 \frac{\mathrm{dR}}{\mathrm{dz}}(2 \mathrm{z})
$$

which can be written in terms of the reflection coefficient $b(p)$

$$
\hat{V}(z)=\frac{2 i}{\pi} \int_{-\infty}^{\infty} p b(p) e^{-i 2 p z} d p .
$$

Recall the expression for the reflection coefficient 


$$
b(p)=\frac{1}{2 i p} \int_{-\infty}^{\infty} e^{i p z^{\prime}} v\left(z^{\prime}\right) \Phi\left(z^{\prime}\right) d z^{\prime}
$$

The Born approximation $\Phi\left(z^{\prime}\right) \simeq e^{i p z^{\prime}}$ results in

$$
b(p) \simeq \frac{1}{2 i p} \int_{-\infty}^{\infty} V\left(z^{\prime}\right) e^{2 i p z^{\prime}} d z^{\prime}
$$

Taking a Fourier transform of both sides leads to

$$
V(z)=\frac{2 i}{\pi} \int_{-\infty}^{\infty} p b(p) e^{-2 i p z} d p
$$

That is the result obtained by Cohen and Bleistein (45). The first order approximation to $\mathrm{V}(\mathrm{z})$ in the Gelfand-Levitan formulation is therefore the Born approximation.

Stickler's inverse procedure entails the solution of the coupled equations

$$
\left\{\begin{array}{l}
-u_{z}^{\prime \prime}-\tilde{q}(z) u_{2}=p^{2} u_{2} \\
\tilde{q}(z)=\frac{2 i}{\pi} \int_{-\infty}^{\infty} p b(p) u_{2}^{2}(z, p) d p
\end{array}\right.
$$

The auxiliary potential $\tilde{q}(z)$ is identical to $V(z)$ and $u_{2}(z, p)$ is the solution that satisfies $u_{2}(z, p)=e^{-i p z}, z \rightarrow-\infty$. To first order assume $u_{2}(z, p)=e^{-i z p}$ and obtain once again, 


$$
\tilde{q}(z)=\frac{2 i}{\pi} \int_{-\infty}^{\infty} p b(p) e^{-2 i p z} d p
$$

\section{2 .3 Algorithm}

The first step is, uf course, the computation of the Fourier transform of the data,

$$
R(z)=\frac{1}{2 \pi} \int_{-\infty}^{\infty} b(p) e^{-i p z} d p,
$$

followed by a straightforward computation of

$$
\hat{V}(z)=-2 \frac{d R}{d z}(2 z)+4 R^{2}(2 z)
$$

Observe that determining the potential at depth $\mathrm{z}$ requires data at depth $2 z$. The truncation of the series leads to a deterioration of the estimate of $\mathrm{V}(\mathrm{z})$ with depth. In particular, since $b(p)$ is integrable, $R(z)$ tends to zero as $z \rightarrow \infty$ (Riemann-Lebesgue theorem) and it follows that $\hat{\mathrm{V}}(\dot{z}) \rightarrow 0$ at depth even when the potential $\mathrm{V}(\mathrm{z})$ tends to a nonzero final potential $\mathrm{V}_{\mathrm{f}}$. 


\subsection{Uniqueness of the Solution}

Suppose there were two solutions of the Gelfand-Levitan equation, $K(z, y)$ and $K^{\prime}(z, y)$

$$
\begin{aligned}
& K(z, y)+R(z+y)+\int_{-y}^{z} K\left(z, z^{\prime}\right) R\left(y+z^{\prime}\right) d z^{\prime}=0 \\
& K^{\prime}(z, y)+R(z+y)+\int_{-y}^{z} K^{\prime}\left(z, z^{\prime}\right) R\left(y+z^{\prime}\right) d z^{\prime}=0
\end{aligned}
$$

Then $\hat{K}(z, y)=K(z, y)-K^{\prime}(z, y)$ satisfies a homogeneous Volterra equation of the second kind

$$
\hat{K}(z, y)=\int_{-y}^{z} \hat{K}\left(z, z^{\prime}\right) R\left(y^{\prime} z^{\prime}\right) d z^{\prime}
$$

Since $R(z)$ is square integrable (cf. Ch.III), it follows (6I) that this equation has only the trivial solution $\hat{K}(z, y)=0$ and therefore the solution of the Gelfand-Levitan equation is unique.

\subsection{Finite Difference Methods}

The natural way to solve the Gelfand-Levitan equation is to discretize it by converting the integral into a sum

$$
\mathrm{K}(\mathrm{n}, \mathrm{m})+\mathrm{R}(\mathrm{n}+\mathrm{m})+\mathrm{h} \sum_{i=-m}^{\mathrm{n}} \mathrm{w}_{i} \mathrm{~K}(\mathrm{n}, \mathrm{i}) \mathrm{R}(\mathrm{m}+\mathrm{i})=0
$$


where $\mathrm{w}_{i}$ are the weights in an appropriate quadrature formula. For the trapezoidal rule, $w_{i}$ is $\frac{1}{2}$ at the end points and 1 in between

$$
\begin{aligned}
& K(n, m)+R(n+m)+h \sum_{i=-m}^{n} K(n, i) R(i+m) \\
& -\frac{1}{2} h(R(0) K+R(n+m) K(n, n))=0
\end{aligned}
$$

and since $R(0)=0$

$$
K(n, m)+h \sum_{i=1-m}^{n} K(n, i) R(i+m)+R(n+m)\left[1-\frac{h}{2}(n, n)\right]=0
$$

To solve for $K(n, n)$, one has to solve the Ware and Aki (21) type matrix equation,

$$
(I+h \hat{R}) K=b
$$

$(I+h \hat{R})$ can be inverted by Gauss elimination. A more efficient algorithm has been presented by Berryman and Greene ( 26 ) who put the equation in the form: 


$$
I+h\left[\begin{array}{c}
R_{1} \\
R_{1} R_{2} \\
\vdots \\
R_{1} R_{2} \ldots \dot{R}_{2 n}
\end{array}\right]\left[\begin{array}{c}
h k(n,-n+1) \\
\vdots \\
h k(n, n-1) \\
1+h k(n, n)
\end{array}\right]=\left[\begin{array}{c}
0 \\
\vdots \\
0 \\
1
\end{array}\right]
$$

where $k(n, m)=\frac{K(n, m)}{1-\frac{h}{2} K(n, n)}$

To obtain the potential at depth $\mathrm{n}-\frac{1}{2}$,

$$
q_{n-\frac{1}{2}}=\frac{2}{h}[K(n, n)-K(n-1, n-1)]
$$

one needs to invert a $(2 n-2) \times(2 n-2)$ matrix for $K(n-1, n-1)$ followed by the inversion of a $(2 n) \times(2 n)$ matrix for $K(n, n)$. In fact, since the object is to reconstruct the potential down to depth $\mathrm{nh}$, a succession of matrices of increasing size have to be inverted. The Berryman and Greene algorithm is similar to the Levinson algorithm for the inversion of Toeplitz matrices (22). The method proceeds by recursion; given the solution of the $(2 n-1) \times(2 n-1)$ system, the solution of the $(2 n) \times(2 n)$ system is generated by using the recursion formulae: 


$$
\left[\begin{array}{l}
f_{i}(2 n) \\
g_{i}(2 n)
\end{array}\right]=\left[\begin{array}{ll}
1 & r_{n} \\
r_{n} & 1
\end{array}\right]=\left[\begin{array}{l}
f_{i}(2 n-1) \\
g_{i}(2 n-1)
\end{array}\right]
$$

The vectors $f(2 n-1)$ and $g(2 n-1)$ are defined by

$$
h\left[\begin{array}{c}
R_{1} \\
R_{1} R_{2} \\
\dot{R_{1}} \ldots \dot{R}_{2 n-1}
\end{array}\right]\left[\begin{array}{c}
f_{2 n-1}(2 n-1) \\
\vdots \\
f_{1}(2 n-1) \\
f_{0}(2 n-1)
\end{array}\right]=\left[\begin{array}{c}
f_{2 n-1}(2 n-1) \\
\vdots \\
g_{1}(2 n-1) \\
g_{0}(2 n-1)
\end{array}\right]
$$

and

$$
h\left[\begin{array}{c}
R_{1} \\
R_{1} R_{2} \\
\cdot \\
\cdot \\
R_{1} \cdots
\end{array}\right]\left[\begin{array}{c}
g_{2 n-1}(2 n-1) \\
\cdot \\
\cdot \\
g_{0}(2 n-1)
\end{array}\right]=\left[\begin{array}{c}
f_{2 n-1}(2 n-1) \\
\cdot \\
\cdot \\
f_{1}(2 n-1) \\
f_{0}(2 n-1)-Q_{2 n-1}
\end{array}\right]
$$

where $Q_{2 n}=Q_{2 n-1}\left(1-r_{n}{ }^{2}\right)$

and $\quad r_{2 n}=h \underset{i=0}{\sum_{i}^{2 n-1}} f_{i}(2 n-1) R_{2 n+1-i} / Q_{2 n-1}$

The recursion starts with $f_{0}(0)=1$ and $g_{0}(0)=\Delta R_{1}$ The solution is then, 


$$
K(n, n)=\frac{2}{h} \frac{1-Q_{2 n-1}\left(1+r_{2 n}\right)}{1+Q_{2 n-1}\left(1+r_{2 n}\right)}
$$

Berryman and Greene have suggested smoothing $R(z)$ to obtain $R(n) \quad(26)$.

$$
R(n)=\frac{1}{h} \int_{n-1}^{n} R(z) d z
$$

which would allow for delta functions in $R(z)$.

\subsection{Coupled Equations Method}

A method to bypass the matrix inversion has been suggested by Kritikos, Jaggard and $\mathrm{Ge}$ who were interested in determining the dielectric permittivity of a slab from reflection measurements at normal incidence. The algorithm was tested numerically on reflection coefficients that could be represented by two and three pole Butterworth filters. The scheme uses in conjunction with the GelfandLevitan equation, the hyperbolic partial differential equation satisfied by $\mathrm{K}(\mathrm{z}, \mathrm{y})$ (cf. Eq. (4.44)).

$$
\frac{\partial^{2} K}{\partial z^{2}}(z, y)-\frac{\partial^{2} K}{\partial y^{2}}(z, y)-2 K(z, y) \frac{d K(z, z)}{d z}=0
$$


For simplicity, a change or coordinates is introduced

$$
\left[\begin{array}{l}
\xi \\
\eta
\end{array}\right]=\frac{1}{2}\left[\begin{array}{rr}
1 & 1 \\
1 & -1
\end{array}\right]\left[\begin{array}{l}
\mathrm{z} \\
\mathrm{y}
\end{array}\right]
$$

and the equations are discretized:

$$
\begin{aligned}
\text { Gelfand-Levitan: } & K_{m, n}+2 h \sum_{i=2}^{m-1} K_{i+n-1, m-i+1} R_{2 i-1} \\
& +\left(1+h K_{m+n-1,1}\right) R_{2 m-1}=0
\end{aligned}
$$

PDE: $\quad K_{m+1, n+1}=K_{m, n+1}+k_{m+1, n}+2 h\left[\left(k_{m+1,1}-K_{m, 1}\right)-1\right] K_{m, n}$

The potential is obtained through

$$
v_{m-1 / 2}=\frac{2}{h}\left(K_{m, 1}-K_{m-1,1}\right)
$$

The point of the method is that $\mathrm{K}_{\mathrm{m}, 1}$ can be computed directly from the Gelfand-Levitan equation without a matrix inversion since the terms within the sum can be generated via the PDE.

\subsubsection{Analysis of Stability}

Although it has not been possible to study the stability of the coupled system of equations, a simplified 
analysis of the PDE may be of interest.

$$
\text { Let } K_{m, n}=W^{m} e^{i k n} \text { be a Fourier component and }
$$

assume that

$$
2 h\left(K_{m+1,1}-K_{m, 1}\right)=h^{2} v_{m}
$$

is known. The PDE becomes

$$
w^{m+1} e^{i k}=w^{m} e^{i k}+w^{m+1}+\left(h^{2} v_{m}-1\right)
$$

The amplification factor is therefore

$$
\left|\frac{W^{m+1}}{w^{m}}\right|=\left|1+\frac{h^{2} v_{m}}{e^{i k}-1}\right|
$$

which is larger than 1 for arbitrarily small step size $h$ or potential $\mathrm{V}_{\mathrm{m}}$ as $\mathrm{k} \rightarrow 0$.

\subsection{Error Estimates}

\subsubsection{Discretization Errors}

The discretization of the Gelfand Levitan equation is accompanied by an error $\varepsilon_{\mathrm{n}, \mathrm{m}}$,

$$
K(n, m)+R(n+m)+h \sum_{i=-m}^{n} w_{i} K(n, i) R(m+i)+\varepsilon_{n, m}=0
$$


For the trapezoidal rule, $\varepsilon=-\left.\frac{h^{2}}{12}(\mathrm{KR})^{\prime}\right|_{-y} ^{z}$

The equation that is actually solved is

$$
\hat{K}(n, m)+R(n+m)+h \sum_{i=-m}^{n} w_{i} \hat{K}(n, i) R(m+i)=0
$$

or, in matrix notation (see equation 5.37 ).

$$
(I+h R) \hat{K}=b
$$

rather than

$$
(I+h R) K=b-\varepsilon
$$

It follows that

$$
(I+h R)(\hat{K}-K)=\varepsilon
$$

For a nonsingular matrix ( $I+h R)$,

$$
\hat{K}-K=(I+h R)^{-1} \varepsilon
$$

Taking norms,

$$
|| \hat{K}-K|| \leq||(I+h R)^{-1}|| \cdot|| \varepsilon||
$$


Assuming that ||$h R||<1$, where the norm is such that ||$I||=1$,

$$
||(I+h R)^{-1}|| \leq \frac{1}{I-h} \Pi|R|
$$

Therefore,

$$
|| \hat{\mathrm{K}}-\mathrm{K}|| \leq \frac{\varepsilon}{1-\mathrm{h}|| \mathrm{R} \mid T}
$$

Using the $\infty$ norm, ||$\varepsilon||_{\infty}=\max \left|\varepsilon_{i}\right|$ and ||$R||_{\infty}=\sum_{i=1}^{2 n}|R(i)|$ (which also equals $\left.|| R\right|_{1}$, the absolute column sum of column $(2 n))$.

$$
|| \hat{K}-K|| \leq \frac{\max |\varepsilon|}{1-h \sum_{i=1}^{2 n}|R(i)|}
$$

Since $|\varepsilon|$ is $0\left(\mathrm{~h}^{2}\right)$ for the trapezoidal rule, the approximate solution $\hat{K}$ converges to the exact solution $K$ as $h \rightarrow 0$. Note that the denominator in (equation 5.62) decreases with increasing depth, hence raising the bound on ||$\hat{K}-K||$.

\subsubsection{Data Errors}

Errors in the plane wave reflection coefficient $r(k z)$ and in its Fourier transform $R(z)$, mean that one is solving for $\hat{K}(n, m)$ in the Gelfand-Levitan equation: 


$$
\hat{K}(n, m)+\hat{R}(n+m)+h \sum_{i=-m}^{n} w_{i} \hat{K}(n, i) \hat{R}(m+i)=0
$$

or in matrix notation,

$$
(I+h \hat{R}) \hat{K}=\hat{b}
$$

which can be written,

$$
(I+h R+h \delta R)(K+\delta K)=(b+\delta b)
$$

where $\hat{R}=R+\delta R$, and $\hat{K}=K+\delta K$.

It follows that

$$
\delta K=\left(I+(I+h R)^{-1} h \delta R\right)^{-1}(I+h R)^{-1}(\delta b-K h \delta R)
$$

Hence,

$$
\begin{aligned}
& || \delta K\|\leq\|(I+h r)^{-1}|| \cdot||\left(I+(I+h R)^{-1} h \delta R\right)^{-1}|| \\
& \cdot(|| \delta b\|+h\| \delta R\|\cdot|| K\|)
\end{aligned}
$$

or,

$$
|| \delta \mathrm{K}|| \leq \frac{1}{1-\mathrm{h}(|| \mathrm{R}||+|| \delta \mathrm{R} \mid)}(|| \delta \mathrm{b}||+\mathrm{h}|| \mathrm{K}|| .|| \delta \mathrm{R}||) .
$$


As the errors in $R(z)$ increase, the bound on ||$\delta K||$ also increases. Moreover, it is clear that as depth increases ||$R||_{\infty}=\sum_{i=1}^{2 n}|R(i)|$ also increases raising the bound on the relative error ||$\delta K|| /|| k||$.

\subsubsection{Errors in the Potential}

The discrete version of the potential yields:

$$
|\delta V(n)| \leq \frac{2}{h}(|\delta K(n, n)|+|\delta K(n-1, n-1)|)
$$

In the absence of data error $\delta R,|\delta V(n)| \rightarrow 0$ as the sampling interval $h \rightarrow 0$ since the quadrature error $\varepsilon$ is $0\left(h^{2}\right)$ (see equation 5-62). 
The derivation of the plane-wave reflection coefficient from pressure measurements for the setup of the Frisk experiment was the subject of Mook's thesis(64). Unfortunately, the attempt by Mook to apply his technique to one set of experimental data yielded reflection coefficients higher than one; such a result is not consistent with physical requirements $(|R|<1)$. It is not yet clear whether Mook's problem lay with improper modeling of the experimental setup, with the imprecision of the data, or with the numerical techniques used to extract the reflection coefficient from the data. It should be noted, however, that the experimental data have yielded, via trial and error methods, excellent models for the acoustic parameters of the seabed(4). This was done by assuming a seabed model, computing the pressure field and matching it up to the observations.

The starting point in our analysis is a layered model of the acoustic profile to be recovered. From this exactly known model, a plane-wave reflection coefficient is generated. The reflection coefficient is what would have been computed from Mook's method, or subsequent improvements to it, for the Frisk experiment on this particular layered seabed. The reflection coefficient is then Fourier trans- 
formed via an FFT to provide the input to the Gelfand-Levitan integral equation in accordance with the theory developed in Chapter IV. The particular case of two half-spaces in contact (step discontinuity) yields a reflection coefficient which can be Fourier transformed analytically. Closed-form solutions for the Born and Improved Born approximations to the inverse solution are also obtained for that case.

More generally, the Gelfand-Levitan equation is solved numerically to yield the potential and velocity profiles.

We have concentrated our efforts on the study of the Gelfand-Levitan inverse method using synthetic data for which the correct answer is known. The comparison of the reconstructed profile with the known original profile allows us to assess the impacts of limited aperture, frequency, profile, noise, density and path loss on the accuracy of the numerical schemes described in Chapter V.

\subsection{Generation of the Reflection Coefficient}

The first step in the evaluation of our approach is the generation of the reflection coefficient. With the exception of the simplest cases, the plane-wave reflection coefficient must be generated numerically. The method used is based on the Thomson-Haskell propagator matrix approach used by Mook(64), with one modification. The plane-wave reflection coefficient is obtained as a function of the vertical wave- 
number $\left(R\left(k_{z}\right)\right)$ rather than as a function of the horizontal wavenumber $\left(R\left(k_{x}\right)\right)$. There are two essential elements of the propagator matrix approach: (a) dividing the acoustic profile into homogeneous layers, and (b) selecting variables that are continuous across interfaces. The latter is accomplished by choosing the pressure $\mathrm{P}(\mathrm{z})$ and vertical component of velocity $U(z)$. Within an isovelocity layer, the field can be decomposed into up and down going waves,

$$
P(z)=P_{+} e^{i k z z}+P_{-} e^{-i k z z}
$$

where $k_{z}$ denotes the vertical wavenumber within the homogeneous layer. Unlike $\mathrm{P}(\mathrm{z}), \mathrm{P}_{+}$and $\mathrm{P}_{-}$are discontinuous at an interface. The normal component of velocity $\mathrm{U}(\mathrm{z})$ is related to $P(z)$ through one of the time-harmonic "telegraph" equations:

$$
\frac{\partial P}{\partial z}=i \omega \rho U
$$

which yields,

$$
U(z)=Y_{n}\left[P_{+} e^{i k z z}-P_{-} e^{-i k z z}\right]
$$

Where $Y_{n}=k_{z_{n}} / \omega \rho_{n}$ is the admittance of the homogeneous layer $\mathrm{n}$. In matrix form, equations (6.2) and (6.3) become: 


$$
\left[\begin{array}{l}
P(z) \\
U(z)
\end{array}\right]=\left[\begin{array}{cc}
e^{i k_{z} z} & e^{-i k_{z} z} \\
Y_{n} e^{i k_{z} z} & -Y_{n} e^{-i k z z}
\end{array}\right]\left[\begin{array}{c}
P_{+} \\
P_{-}
\end{array}\right]
$$

When $P(z)$ and $U(z)$ are known at point $z_{1}$ of the layer, one can deduce the up and down going components $\mathrm{P}_{+}$and $\mathrm{P}_{-}$by inverting the matrix in (6.4)

$$
\left[\begin{array}{l}
P_{+} \\
P_{-}
\end{array}\right]=\left[\begin{array}{cc}
e^{i k_{z} 1} & e^{-i k_{z^{z}} 1} \\
-Y_{n} e^{-i k_{z} z_{1}} & -Y_{n} e^{-i k_{z} z_{1}}
\end{array}\right]^{-1}\left[\begin{array}{l}
P\left(z_{1}\right) \\
U\left(z_{1}\right)
\end{array}\right]
$$

(Within a homogeneous layer $\mathrm{P}_{+}$and $\mathrm{P}_{-}$are, of course, constants.) One can then determine $P(z)$ and $U(z)$ at another point, say $\mathrm{z}_{2}$, within the layer by substituting (6.5) into the right-hand side of $(6.4)$ :

$$
\left[\begin{array}{l}
\mathrm{P}\left(\mathrm{z}_{1}\right) \\
\mathrm{U}\left(\mathrm{z}_{1}\right)
\end{array}\right]=\left[\begin{array}{ll}
\operatorname{cosk}_{\mathrm{z}}\left(\mathrm{z}_{1}-\mathrm{z}_{2}\right) & \frac{i}{Y_{n}} \operatorname{sink}_{z_{1}}\left(\mathrm{z}_{1}-\mathrm{z}_{2}\right) \\
\text { iY } \operatorname{sink}_{\mathrm{z}}\left(\mathrm{z}_{1}-\mathrm{z}_{2}\right) & \operatorname{cosk}_{\mathrm{z}}\left(\mathrm{z}_{1}-\mathrm{z}_{2}\right)
\end{array}\right]\left[\begin{array}{l}
\mathrm{P}\left(\mathrm{z}_{2}\right) \\
\mathrm{U}\left(\mathrm{z}_{2}\right)
\end{array}\right]
$$


or, introducing the propagator matrix $\Phi$

$$
\left[\begin{array}{l}
P\left(z_{1}\right) \\
U\left(z_{1}\right)
\end{array}\right]=\Phi_{1}\left(z_{2}-z_{1}\right)\left[\begin{array}{l}
P\left(z_{2}\right) \\
U\left(z_{2}\right)
\end{array}\right] \text {. }
$$

One can now proceed from one layer to another by integration,

$$
\left[\begin{array}{l}
\mathrm{P}\left(\mathrm{z}_{0}\right) \\
\mathrm{U}\left(\mathrm{z}_{0}\right)
\end{array}\right]=\underbrace{\Phi_{1} \Phi_{2} \cdots \Phi_{n}}_{\Phi}\left[\begin{array}{c}
\mathrm{P}\left(\mathrm{z}_{\mathrm{n}}\right) \\
\mathrm{U}\left(\mathrm{z}_{\mathrm{n}}\right)
\end{array}\right]
$$

Where the $\Phi_{i}$ 's depend on the parameters of the material making up the respective layers. Mook (64) has found that the computations can be improved by modifying $(6.8)$ to

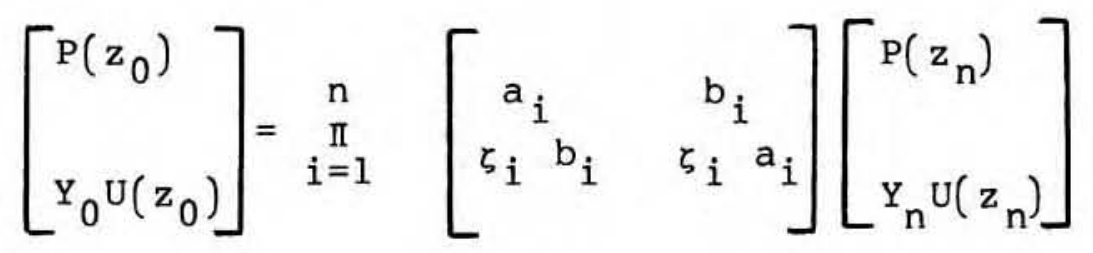

in which

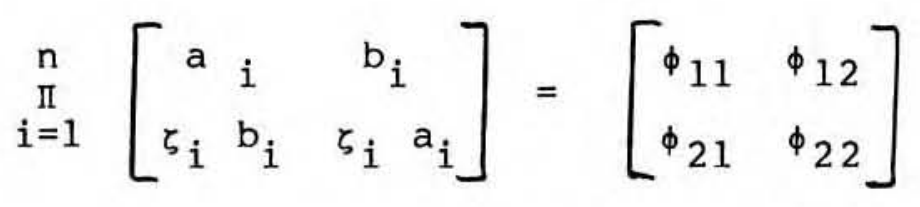


and where

$$
\begin{aligned}
& \zeta_{i}=\frac{Y_{n-1}}{Y_{n}} \text { is a normalized admittance } \\
& a_{i}=\cos k_{z_{i}} h_{i} \\
& b_{i}=-i \sin k_{z_{i}} h_{i} \\
& h_{i}=\text { thickness of }{ }^{t h} \text { layer } \\
& k_{z i}=\sqrt{k_{n}^{2}-\left(k_{0}^{2}-k_{z_{0}}^{2}\right)} .
\end{aligned}
$$

This has the effect of giving the two components $P(z)$ and YU(z) similar scales of magnitude.

Now, the reflection coefficient $R\left(k_{z}\right)$ at the top interface is defined as

$$
R\left(k_{z}\right)=\frac{P-0}{P+0}
$$

Therefore by introducing (6.5) into (6.9) one obtains

$$
\begin{gathered}
{\left[\begin{array}{l}
\mathrm{P}_{+0} \\
\mathrm{P}_{-0}
\end{array}\right]=\frac{1}{2}\left[\begin{array}{rr}
1 & 1 \\
1 & -1
\end{array}\right]\left[\begin{array}{ll}
\phi_{11} & \phi_{12} \\
\phi_{21} & \phi_{22}
\end{array}\right]\left[\begin{array}{l}
\mathrm{P}_{\left(\mathrm{z}_{\mathrm{n}}\right)} \\
\zeta_{\mathrm{n}} \mathrm{U}\left(\mathrm{z}_{\mathrm{n}}\right)
\end{array}\right]} \\
-109-
\end{gathered}
$$


To compute the ratio in $(6.10)$, a relation (boundary condition) is needed between $P\left(z_{n}\right)$ and $U\left(z_{n}\right)$. That relation is obtained by noticing that in the $(n+1)^{\text {st }}$ layer, one has only down going waves. Therefore,

$$
U_{n+1}=\frac{1}{\zeta_{n+1}} P\left(z_{n+1}\right)
$$

and, since $P_{n+1}=P\left(z_{n}\right)$, one has

$$
\left[\begin{array}{c}
P_{+0} \\
P_{-0}
\end{array}\right]=\frac{1}{2}\left[\begin{array}{ll}
\phi_{11}+\phi_{21} & \phi_{12}+\phi_{22} \\
\phi_{11}-\phi_{21} & \phi_{12}-\phi_{22}
\end{array}\right]\left[\begin{array}{c}
1 \\
\zeta_{n+1}
\end{array}\right] P_{n+1}
$$

which yields the reflection coefficient

$$
R\left(k_{z}\right)=\frac{P_{-0}}{P_{+0}}=\frac{\phi_{11}-\phi_{21}+\zeta_{N+1}\left(\phi_{12}-\phi_{22}\right)}{\phi_{11}+\phi_{21}{ }^{+} \zeta_{N+1}\left(\phi_{12}{ }^{+} \phi_{22}\right)} \text {. }
$$

Mook (64) has also found it advantageous to scale the layer propagation matrices so that the largest element value in a given layer matrix is 1 .

The values of admittance for three terminations are readily identified: hard bottom $\left(Y_{n+1}=0\right)$, soft bottom 
$\left(Y_{n+1}=\infty\right)$, and isovelocity termination $\left(Y_{n+1}=Y_{n}\right)$.

Although of theoretical interest, and of possible applicability to other situations, the soft bottom case has no application in the ocean bottom problem discussed here.

The division of the acoustic profile into homogeneous layers is done in a way akin to quadrature formulae in numerical integration using thinner, closely-spaced, layers in regions of rapid change in the acoustic parameters and thicker, wider-spaced layers in regions of slow change. We have found that the common rule of thumb, ten layers per wavelength, although satisfactory in general, is probably too conservative. We have found that for complicated profiles, where the reflection coefficient was required for a large number of values of $\mathrm{k}_{\mathrm{z}}$, the computation of the plane-wave reflection coefficient constituted the most time-consuming step in modeling the whole inversion procedure. Clearly, this step, is inherent only in our analytical evaluation of the inversion problem; when reflection coefficients are being processed from measurements, this step will be eliminated.

In order to test our inverse procedure, we selected a few representative profiles which are defined as follows: 
(a) Half-Space

The half-space profile is defined by a velocity profile $c(\mathrm{z})$,

$$
\begin{array}{rlrl}
c(z) & =c_{0}, & z<0 \\
& =c_{1}, & z & >0
\end{array}
$$

The reflection coefficient for this profile and its Fourier transform were obtained analytically in Section $(6.2)$

(b) Window Profile

Although of no direct application in ocean bottom acoustics, the window profile defined by

$$
\begin{array}{rlrl}
c(z) & =c_{0}, & & z<0 \\
& =c_{1}, & & 0<z<L \\
& =c_{0}, & z>L
\end{array}
$$

is a standard example used to test inverse procedures. The window profile is of particular interest in electromagnetics (dielectric slabs). 


\section{(c) Stickler Profile}

Stickler(7) chose a twice continuous function to test the Deift-Trubowitz inverse method,

$$
\begin{array}{rlrl}
c(z) & =c_{0}, & & z<0 \\
& =c_{0}+\left(c_{1}-c_{0}\right)\left(3\left(\frac{z}{L}\right)^{2}-2\left(\frac{z}{L}\right)^{3}\right), & & 0<z<L \\
& =c_{1}, & L<z .
\end{array}
$$

This profile does not support a trapped mode at any frequency.

Stickler generated the associated reflection coefficient via the Riccati equation, while we used the Thomas-Haskell procedure outlined earlier in this section. An example of the stickler profile is shown in Fig. 10 .

(d) Frisk Profile

This velocity profile is based on the results obtained by Frisk in his deep ocean bottom experiments (4)

$$
\begin{aligned}
c(z) & =c_{0}, & & z<0 \\
& =c_{0}-\left(c_{0}-c_{1}\right) z, & & 0<z z 1 \\
& =c_{1}+\gamma z, & & 1<z<L \\
& =c_{2}, & & z>L
\end{aligned}
$$


Typical parameters used are: $\mathrm{c}_{0}=1540 \mathrm{~m} / \mathrm{s}, \mathrm{c}_{1}=$ $1515 \mathrm{~m} / \mathrm{s}, c_{2}=1655 \mathrm{~m} / \mathrm{s}, \gamma=.97$, and $\mathrm{L}=145 \mathrm{~m}$ (see Fig. 11).

In spite of the low velocity zone near the ocean-bottom interface, this profile does not support trapped modes at the frequency used for the examples $(25 \mathrm{~Hz})$.

Examples of reflection coefficients are shown in the amplitude and phase diagrams of Fig. 18. Note that as we have shown in Chapter III for the uniform density case, the amplitude goes asymptotically to zero as the vertical wavenumber goes to infinity.

\subsection{Case of a Step Discontinuity in Potential}

We have been able to obtain analytically the Fourier transform of the reflection coefficient in the case of a step discontinuity in potential, that is, in the case where the ocean bottom is a homogeneous half-space. $\left(V(z)=V_{0}\right.$ for $z>0)$.

The reflection coefficient at a step discontinuity in potential is 


$$
\begin{aligned}
r\left(k_{z}\right) & =\frac{k_{z}-\sqrt{-v_{0}+k_{z}^{2}}}{k_{z}+\sqrt{-v_{0}+k_{z}^{2}}} \\
& =\frac{\left[k_{z}-\sqrt{-v_{0}+k_{z}^{2}}\right]^{2}}{v_{0}}
\end{aligned}
$$

The Fourier transform of $r\left(k_{z}\right)$ is

$$
R(z)=\frac{1}{2 \pi} \int_{-\infty}^{\infty} r\left(k_{z}\right) e^{-i k_{z} z} d k_{z}
$$

$$
=\frac{i}{2 \pi i} \int_{-i \infty}^{i \infty} \frac{\left(s-\sqrt{v_{0}+s^{2}}\right)^{2}}{v_{0}} e^{s z} d s
$$

which in this form can be identified as a known inverse Laplace transform $(65)$,

$$
R(z)=-\frac{2}{z} J_{2}\left(\sqrt{\nabla_{0}} z\right)
$$

Thus, in this particular case, one can proceed to the Gelfand-Levitan procedure with an input which is as accurate as the computation of the Bessel function, and therefore, this approach removes any inaccuracies which may be 
introduced either in the computation of the reflection coefficient or in that of its Fourier transform.

Figs. 12a,b,c represent the reflection coefficient of eq. 6.15 and its Fourier transform for a half-space (eq. 6.17).

As a matter of fact, we have illustrated in Figs. 13 and $14 \mathrm{~b}$ reconstructions of the step discontinuity using the exact analytical expression (eq. 6.17) for the Fourier transform of the reflection coefficient and the numerically devised transform in accordance with the method discussed in Section 6.1. It can be seen that our reflection coefficient method yields results as accurate as the Bessel function expression.

6.2.1 Approximate Solution of the Gelfand-Levitan Equation for a Step Discontinuity

Continuing with the case of a homogenous half-space, or step discontinuity, the "Improved Born" approximation presented in Chapter $\mathrm{V}$ can be now readily obtained in terms of Bessel functions 


$$
\hat{V}(z)=-2 \frac{d R}{d z}(2 z)+4 R^{2}(2 z)
$$

$$
\begin{array}{r}
=4 \frac{\sqrt{\mathrm{V}_{0}}}{\mathrm{z}} \mathrm{J}_{1}\left(2 \sqrt{\mathrm{V}_{0} \mathrm{z}}\right)-\frac{6}{\mathrm{z}^{2}} \mathrm{~J}_{2}\left(2 \sqrt{\mathrm{V}_{0} \mathrm{z}}\right)-\frac{4}{\mathrm{z}} \mathrm{J}_{2}^{2}\left(2 \sqrt{\mathrm{V}_{0} \mathrm{z}}\right) . \\
\text { Born } \\
\text { Correction Term } \\
\text { Improved Born } .
\end{array}
$$

From the asymptotic behavior of the Bessel functions, we know that,

$$
\mathrm{J}_{1}(\zeta)_{\zeta \rightarrow \infty} \sqrt{\frac{2}{\pi \zeta}} \cos \left(\zeta-\frac{3 \pi}{4}\right)
$$

and

$$
J_{2}(\zeta)_{\zeta}+\underset{\frac{2}{\pi \zeta}}{\cos \left(\zeta-\frac{5 \pi}{4}\right)} \text {. }
$$

Hence, we can deduce the asymptotic behavior of the reconstructed potential,

$$
\hat{V}(z) \underset{z+\infty}{\rightarrow} \operatorname{as}\left(z^{-3 / 2}\right)
$$

which recalls the limitation of the Improved Born approximation, already mentioned in Chapter $\mathrm{V}$, that $\mathrm{V}(\mathrm{z}) \underset{\mathrm{z} \rightarrow \infty}{\rightarrow} \underset{\text {, even }}{\rightarrow}$ in the case of a finite terminal potential. 
On the other hand, the asymptotic behavior of the Bessel functions near the origin is

$$
\begin{aligned}
\mathrm{J}_{1}(\zeta)_{\zeta} \rightarrow 0 & \frac{1}{2} \zeta
\end{aligned}
$$

$$
\begin{array}{r}
J_{2}(\zeta)+0 \\
\zeta+0 \\
\frac{1}{8} \zeta^{2}
\end{array}
$$

and therefore, substituting in (6.18) the reconstructed potential $\mathrm{V}(\zeta)$ tends to the exact potential near the origin,

$$
\hat{V}(z)+v_{0}
$$

At the origin, the "Improved Born" correction term is zero, but its contribution to the accuracy of the results becomes progressively more important as $z$ increases.

We illustrate the Improved Born approximation in Fig. 12d along with the Standard Born approximation and the Correction Term. The substantial improvement due to the Correction Term in (6.18) over the standard Born approximation is clearly visible in the half-space case. Moreover, our Improved Born approximation results in a more accurate reconstruction of the acoustic velocity profile to further depth. The other features of the approximation are also visible, i.e., excellent reconstruction near the origin and 
deterioration of the reconstructed velocity with depth (the potential $\mathrm{V} \rightarrow 0$ as $\mathrm{z} \rightarrow \infty$ means that $\mathrm{c}(\mathrm{z}) \rightarrow \mathrm{c}_{0}$, the water acoustic velocity as $z+\infty)$. The Improved Born is also illustrated in the case of a stickler profile in Figs. 28 and 29 .

\subsection{Fourier Transform of the Reflection Coefficient}

The Gelfand-Levitan algorithm requires as an input the Fourier transform, $R(z)$, of the reflection coefficient $r\left(k_{z}\right)$,

$$
R(z)=\frac{1}{2 \pi} \int_{-\infty}^{\infty} r\left(k_{z}\right) e^{-i k_{z} z} d k_{z}
$$

which requires a knowledge of $r\left(k_{z}\right)$ over the whole line $-\infty<\mathrm{k}_{\mathrm{z}}<\infty$. But the symmetry property demonstrated in Chapter III (eq. 3.6),

$$
r\left(-k_{z}\right)=r^{*}\left(k_{z}\right)
$$

reduces the requirement to a knowlege of $r\left(k_{z}\right)$ over the halfline $0<\mathrm{k}_{\mathrm{z}}<\infty$. It follows that

$$
R(z)=\frac{1}{2 \pi} \int_{0}^{\infty} r\left(k_{z}\right) e^{-i k z^{z}} d k_{z}+\text { complex conjugate }
$$

is real, and therefore all the quantities involved in the Gelfand-Levitan algorithm are also real. 
Theoretically, $r\left(k_{z}\right)$ can be calculated for a given acoustic model over the range $0<\mathrm{k}_{\mathrm{z}}<\infty$. In practice, samples of $r\left(k_{z}\right)$ are given over a finite range $a<k_{z}<b$, and presumably this range is restricted to real angles $0<k_{z}<k_{0}$, where $k_{z}=0$ corresponds to grazing incidence and $k_{z}=k_{0}$ (the water wavenumber) corresponds to vertical incidence. It is useful to study the behavior of the Fourier transform under different restrictions imposed upon the knowledge of the reflection coefficient such as limited angular aperture and different sampling densities.

\subsubsection{Fast Fourier Transform}

The computation of the FFT for reflection coefficients $r\left(k_{z}\right)$ computed over $0<k_{z}<a$, including the case where a $>k_{0}$ (corresponding to complex angles of incidence), does not present difficulties. The adopted algorithm uses time decomposition with input bit reversal(66). In fact, for the acoustic profiles tested, $a=2$ or $3 k_{0}$ was sufficient as the asymptotic decay of the reflection coefficient with increasing $k_{z}$ was even more rapid than the $\left(1 / k_{z}{ }^{2}\right)$ derived in Chapter III. The errors in the imaginary part of the FFT, which are of the order of $10^{-8}$ (single precision), are negligible, and therefore the real part of the FFT and its amplitude are interchangeable on the plotted results. 


\subsubsection{Limited Angular Aperture}

Since it is not practical to have the sampling process cover the entire non-zero portion of $r\left(k_{z}\right)$, it is useful to study the effect of limited angular aperture, i.e., $r\left(k_{z}\right)$ given over the finite range $a<k_{z}<b$, on $R(z)$, the Fourier transform of $r\left(k_{z}\right)$. The Fourier transform is affected by the nature of $r\left(k_{z}\right)$ and by the degree of truncation.

For $\mathrm{N}$ sampling points spaced $\Delta \mathrm{k}_{\mathrm{Z}}$ apart, the total sampling interval $\mathrm{T}$ covered is $\mathrm{N} \cdot \Delta \mathrm{k}_{\mathrm{Z}}$. This corresponds to an angular aperture of $\alpha=\sin ^{-1}\left(\frac{\mathrm{N} \Delta \mathrm{k}_{\mathrm{z}}}{\mathrm{k}_{0}}\right)$, when $\mathrm{k}_{\mathrm{z}}=0$ is within the known aperture (i.e., 90 $\left.>0>\cos ^{-1}\left(\mathrm{~N} \Delta \mathrm{k}_{\mathrm{z}} / \mathrm{k}_{0}\right)\right)$. Note that for $\mathrm{T}>\mathrm{k}_{0}, \cos \left(\mathrm{T} / \mathrm{k}_{0}\right)>1$, and the aperture includes all real angles plus complex angles. Now, the Fourier transform of $r\left(k_{z}\right)$ can be written

$$
\begin{aligned}
R(z) & =\frac{1}{2 \pi} \int_{0}^{T} r\left(k_{z}\right) e^{-i k_{z} z} d k_{z}+\frac{1}{2 \pi} \int_{T}^{\infty} r\left(k_{z}\right) e^{-i k_{z} z} d k_{z} \\
& =R_{1}(z)+R_{2}(z) .
\end{aligned}
$$

$R_{1}(z)$ is the part of $R(z)$ that is approximated by the discrete Fourier transform ( $N$ samples).

$R_{2}(z)$ represents the error incurred due to the limited angular aperture. The energy lost in the process, 


$$
E=\int_{T}^{\infty}\left|R_{2}(z)\right|^{2} d z
$$

can be computed on synthetic examples. Due to the precipitous drop in $r\left(k_{z}\right)$ beyond $k_{C}$, the wavenumber corresponding to the critical angle $\theta_{c}=\sin ^{-1}\left(\frac{c_{0}}{c_{f}}\right)$, and due to the asymptotic behavior of $r\left(k_{z}\right)$ for large $k_{z}$, it is readily shown on computer simulations that a knowledge of $r\left(k_{z}\right)$ over real angles is adequate for most cases. In fact, there is little change in the reconstructed profile as more angles are included beyond the real ones. On the other hand, as the angular aperture is restricted, the profile reconstruction, via the Gelfand-Levitan method, produces a smoothed out version of the original profile. This low-pass filtering phenomenon is best understood by interpreting the effect of a finite aperture as a low-pass filtering of the original velocity profile. This may be seen from the Born approximation eq. (5.26),

$$
\hat{V}(z)=\frac{2 i}{\pi} \int_{-\infty}^{\infty} k_{z} r\left(k_{z}\right) e^{-2 i k_{z} z} d k_{z}
$$

where $\hat{\mathrm{V}}(\mathrm{z})$ and $\mathrm{k}_{\mathrm{z}} r\left(\mathrm{k}_{\mathrm{z}}\right)$ form a Fourier transform pair; windowing $r\left(k_{z}\right)$ signifies low-pass filtering $\hat{V}(z)$ and therefore yields a smoothed out velocity profile. The Gelfand-Levitan reconstruction can then be interpreted as an unfiltered, faithful reconstruction of the smoothed out original. Severe degradation of the reconstruction is 
observed when the angle of incidence is restricted to $90^{\circ}>\theta>\theta_{C}$ (angular aperture $\left.\alpha<\left(90^{\circ}-\theta_{C}\right)\right)$. This is easily understood, as the critical angle region contributes substantially to the reflection coefficient. A cursory examination of reflection profiles will demonstrate this point.

A series of figures displays the progressive deterioration in profile reconstruction as the angular range is restricted. The evolution from $\mathrm{Fig} .19\left(\mathrm{k}_{\mathrm{z} \text { max }}=.512\right.$, complex angle of incidence $)$ to Fig. $20\left(k_{z_{\max }}=0.128\right)$, and Fig.

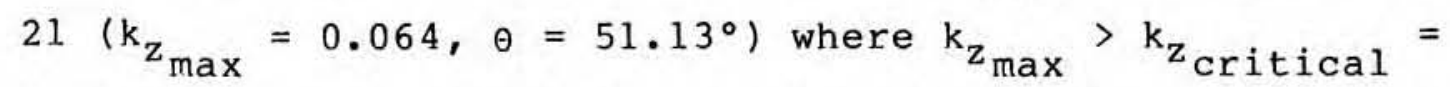
$0.0373\left(\theta_{C}=68.5^{\circ}\right)$ shows that only small changes take place and that these changes are confined for the most part to the velocity drop region near the ocean bottom interface. However, as the angles are further restricted to beyond the critical angle $\left(\mathrm{k}_{\mathrm{z}_{\max }}<\mathrm{k}_{\mathrm{z}_{\text {critical }}}\right)$ major changes do occur as seen in Fig. $22\left(\mathrm{k}_{\max }=.032, \theta=71^{\circ}\right)$ and Fig. $23\left(\mathrm{k}_{\max }=\right.$ $\left.0.016, \theta=80.9^{\circ}\right)$. The examination of the impact of limited angular aperture in this case leads us to expect, in practice, a good reconstruction of acoustic velocity profiles from reflection data restricted to real angles $\left(0<\theta<90^{\circ}\right)$. 


\section{3 .3 Sampling}

The sampling rate to be chosen is governed by three competing considerations:

(a) Adequate sampling in the $\mathrm{k}_{\mathrm{z}}$-domain to avoid aliasing in the Fourier transform of $r\left(k_{z}\right)$.

(b) Adequate sampling in the $z$-domain to obtain a stable and accurate Gelfand-Levitan numerical reconstruction.

(c) Adequate sampling in the $z$-domain to obtain the necessary resolution in the reconstructed profile.

Each of these points is discussed next.

\section{(a) Aliasing}

Since the Fourier transform of the reflection coefficient, as is the rule with spectra of transients, tends to be smooth and approaches zero asymptotically as $k_{z}$ increases to infinity, the sampling interval $\Delta \mathrm{k}_{\mathrm{z}}$ is chosen so that essentially all, rather than all, the spectral content of the waveform is contained below $1 /\left(2 \Delta \mathrm{k}_{\mathrm{z}}\right)$. For a box-like reflection coefficient of width $2 \cdot k_{\text {critical }}$ where $k_{\text {critical }}$ corresponds to $\theta_{\text {crit, }}$ the bandwidth is proportional to $1 /\left(2 \mathrm{k}_{\text {critical }}\right)$ which indicates that an appropriate sampling interval should be a fraction of $k_{c r i t i c a l}$. One should note 
however, that in the context of an actual experiment, the choice of $\Delta \mathrm{k}_{\mathrm{z}}$ is not as straightforward. The reflection coefficient $r\left(k_{z}\right)$ is obtained from $r\left(k_{r}\right)$, the reflection coefficient as a function of horizontal wavenumber $k_{r}$,

$$
k_{r}^{2}+k_{z}^{2}=k_{0}^{2}
$$

The sampling interval in $k_{r}$ is determined by the maximum distance, D, between source and receiver during the experiment

$$
\Delta k_{r}=\frac{\pi}{D}
$$

It is clear that a uniform sampling in $\mathrm{k}_{\mathrm{r}}$ does not lead to a uniform sampling in $\mathrm{k}_{\mathrm{z}}$,

$$
\frac{d k_{z}}{d k_{r}}=-\tan \theta_{\text {inc }} \text {, }
$$

which also shows that the problem is particularly severe near $\theta_{\text {inc }}=\pi / 2$ (grazing). On the other hand $r\left(k_{z}\right)$ near grazing is well known, $r\left(k_{z}\right) \simeq-1$. A useful way to look at the problem is to represent the dispersion relation in the $\left(k_{r}-k_{z}\right)$ plane which clearly shows the increase in $\Delta k_{z}$ as $k_{r}$ goes from 0 to $k_{0}$. 
(b), (c) Sampling in the z-domain

The sampling interval in wavenumber space, $\Delta \mathrm{k}_{\mathrm{z}}$, is not chosen through aliasing considerations alone. The other consideration is the resulting resolution in the $z$-domain imposed by the total sampling interval $\mathrm{T}$,

$$
\Delta z=\frac{2 \pi}{T}
$$

where $\mathrm{T}=\mathrm{N} \Delta \mathrm{k}_{\mathrm{z}}$

A decrease in $\Delta z$ can be effected either by a decrease in the sampling interval in the wavenumber domain $\Delta \mathrm{k}_{\mathrm{z}}$ or by an increase in the number of points $N$. It is easier to resort to the latter method as the reflection coefficient due to its rapid decrease for large vertical wavenumbers can be conveniently padded with zeros. Moreover, the size of $\Delta \mathrm{k}_{\mathrm{z}}$, which can be varied in a synthetic experiment, is usually fixed in an actual experiment.

An examination of the reconstructions of a half-space of profile, Figs. 14-17, reveals that excellent reconstructions can be achieved for appropriate choices of $\mathrm{N}$ and $\Delta \mathrm{z}$. A window reconstruction is shown in Fig. 24. The main effect of a decrease in $\Delta \mathrm{z}$ is that adequate reconstruction of the acoustic profile is possible to a greater depth. It is also true that for given sampling intervals $\Delta \mathrm{k}_{\mathrm{z}}$ and $\Delta \mathrm{z}$, an increase in the height of the velocity jump at the seafloor 
results in a deterioration of the reconstruction with depth. That is evident for a half-space by comparing Fig. $14 \mathrm{~b}$ and Fig. 17 and for the Improved Born approximation by analyzing eq. (6.18).

\subsection{Frequency Scaling of the Reflection Coefficient}

Although the experiment we are analyzing is monochromatic, it is important, for a proper choice of operating frequency, to study the behavior of the plane-wave reflection coefficient with frequency. For the simplest acoustic medium, a homogeneous half-space, the reflection coefficient is independent of frequency. As soon as a spatial scale is introduced in the acoustic medium, by inserting a layer for instance, the reflection coefficient becomes frequency dependent. This dependence, which can be expressed through the continued fraction expression of eq. (3.25), is at the heart of the Ware and Aki inverse method. In this section we show that the solution of a high frequency problem is equivalent to the solution of a scaled problem at a lower frequency.

The one-dimensional Schrodinger equation,

$$
\left(\frac{d^{2}}{d z^{2}}+k_{z}^{2}\right) \phi(z)=V(z) \phi(z)
$$

gives rise to the reflection coefficient

$$
R\left(k_{z}\right)=\int_{-\infty}^{\infty} \frac{e^{i k} z^{\prime}}{2 i k z} V\left(z^{\prime}\right) \phi\left(z^{\prime}\right) d z^{\prime}
$$


At a different angular frequency $\omega^{\prime}=\alpha \omega_{0}$, with corresponding water wavenumber $\mathrm{k}^{\prime}=\alpha \mathrm{k}_{0}$, the Schrodinger equation becomes

$$
\left(\frac{\mathrm{d}^{2}}{\mathrm{dz}^{2}}+\alpha^{2} \mathrm{k}^{2}{ }^{2}\right) \phi^{\prime}(\mathrm{z})=\mathrm{V}^{\prime}(\mathrm{z}) \phi^{\prime}(\mathrm{z})
$$

where $V^{\prime}$ represents the potential at the new frequency $\omega^{\prime}$. Now, the potential is frequency dependent,

$$
v_{0}(z)=k_{0}^{2}\left(1-n^{2}\right)
$$

in which $n$, the index of refraction is a function of depth $z$. Therefore,

$$
V^{\prime}(z)=\alpha^{2} V_{0}(z)
$$

yields the Schrodinger equation

$$
\left(\frac{d^{2}}{d z^{2}}+\alpha^{2} k_{z}{ }^{2}\right) \phi^{\prime}(z)=\alpha^{2} v_{0}(z) \phi^{\prime}(z)
$$

The change of variable $z^{\prime}=\alpha z$ restores the original Schrodinger equation (6.31) albeit with a "stretched" version of the original potential,

$$
\left(\frac{d^{2}}{d z^{\prime 2}}+k_{z}^{2}\right) \phi^{\prime}\left(z^{\prime}\right)=v_{0}\left(z^{\prime} / \alpha\right)_{\phi^{\prime}}\left(z^{\prime}\right)
$$


The corresponding reflection coefficient is now

$$
R^{\prime}\left(k_{z}\right)=\int_{-\infty}^{\infty} \frac{e^{i k z^{\prime}}}{2 i k z} v_{0}\left(z^{\prime} / \alpha\right) \phi^{\prime}\left(z^{\prime}\right) d z^{\prime}
$$

The reflection coefficients at the experimental

frequencies $\omega$ and $\omega^{\prime}$ are therefore identical for a given wavenumber $k_{z}$ if

$$
v_{0}(z)=v_{0}\left(z^{\prime} / \alpha\right)
$$

One can therefore conclude that a high frequency experiment $\left(\omega^{\prime}>\omega^{\prime}\right)$ is equivalent to a low frequency experiment with a stretched profile $(\alpha>1)$. One can therefore compare the reconstruction of a given profile at two frequencies, say $\omega$ and $\omega^{\prime}=\alpha \omega$, by comparing the reconstruction at a single frequency $\omega$ of the given profile with its stretched version (stretch factor $\alpha$ ). Now, it is a numerical fact, as seen in previous examples, that profile reconstruction via the Gelfand-Levitan algorithm deteriorates with depth. It is therefore clear, at least for simple profiles, such as a step (half-space) or a window (layer), that a lower experimental frequency entails deeper reconstruction as shown in Fig. 30 . That holds for more complicated profiles, and we therefore conclude from a frequency scaling point of view that the lowering of the experimental frequency allows for deeper profile reconstruction without noticeable effects on the reconstruction of the detailed variations of the profile. 


\subsection{Profile Reconstruction in the Presence of Density}

\section{Variations}

Although the focus of this thesis has been on the reconstruction of the acoustic profile in the presence of velocity variations at a single frequency, it is useful here to show how one can extend the method to the retrieval of the velocity in the presence of density variations and also to recover the density profile.

We have shown in Chapter II (eq. (2.5)) that in the presence of smooth density variations, a change of variables retained the governing Helmholtz and Schrodinger equations with an attending redefinition of the index of refraction to account for variations in the density $\rho$,

$$
n^{\prime}=n^{2}+k_{0}^{-2}\left(\frac{1}{2 \rho} \nabla^{2} \rho-\frac{3}{4}\left(\frac{1}{\rho} \nabla \rho\right)^{2}\right) .
$$

The density dependent potential to be reconstructed is now,

$$
V^{\prime}=k_{0}^{2}\left(1-n^{\prime 2}\right)
$$

A single frequency experiment can only hope to reconstruct $n^{\prime}(z)$. To recover $n(z)$ (and therefore the velocity, $c(z)$ ) and $\rho(z)$ one needs to carry out the experiment at two frequencies $\omega_{1}$ and $\omega_{2}$ with water wavenumbers $k_{0}$ and $k_{1}$, respectively. The associated potentials are then 


$$
v_{1}(z)=k_{1}^{2}\left(1-n^{\prime 2}\right)
$$

and

$$
v_{2}(z)=k_{2}^{2}\left(1-n^{\prime}{ }_{2}^{2}\right) .
$$

These potentials can be reconstructed by the application of the Gelfand-Levitan algorithm to the corresponding reflection coefficients. One can then obtain the difference of the potentials,

$$
v_{1}-v_{2}=\left(k_{1}^{2}-k_{2}^{2}\right)\left(1-n^{2}\right),
$$

and therefore retrieve the velocity dependent index of refraction $n(z)$,

$$
n(z)=\frac{\left(v_{1}-v_{2}\right)}{k_{1}^{2}-k_{2}^{2}}-1
$$

and subsequently reconstruct the acoustic velocity profile,

$$
c(z)=\frac{c_{0}}{n(z)} \text {. }
$$

One of our numerical computations, was to conduct the two-frequency procedure on the profile of Figure 10 . The reconstruction of $\mathrm{c}(\mathrm{z})$ is shown in Figure $30 i$. The reconstruction features in this case of $c(z)$ are similar to the 
constant density case. The density as function of $\mathrm{z}$ could in turn be recovered by solving the differential equation ( 6.40$)$ for $p(z)$ given $n(z)$.

Density discontinuities invalidate the asymptotic behavior of the reflection coefficient presented in Chapter III. In fact, the reflection coefficient is generally not integrable, and one has to introduce generalized functions in its Fourier transform. The attending difficulties and their resolution in the Gelfand-Levitan algorithm have not been studied here.

\subsection{Acoustic Attenuation}

The study of the attenuation of acoustic waves in marine sediments has been studied recently by Rajan and Frisk(67) who proposed a perturbative inverse method for the recovery of the attenuation data from reflection data. Rajan has successfully inverted for the acoustic attenuation profile given the reconstructed velocity profile we had obtained through the Gelfand-Levitan algorithm(68). Here, we shall look at the effects of intrinsic attenuation on the reconstructed acoustic profile.

Although our formulation of the model of a Frisk experiment does not include attenuation, it is possible to posit a lossy acoustic profile, generate the corresponding reflection coefficient and then run it through the Gelfand-Levitan 
algorithm. As expected, for small loss $(\alpha \sim 0.001-0.005$ $\mathrm{dB} / \mathrm{m})$, the reflection coefficient, its Fourier transform, and the reconstructed profile are little affected by the perturbation (see Figs. 25a, 25b, 26, 27). As loss increases $(\alpha \sim 0.01 \mathrm{~dB} / \mathrm{m})$, the reconstructed profile deteriorates rapidly. It should be pointed out that the lower values of intrinsic attenuation prevail in the sediments in the abyssal plain at the frequencies of interest $(220 \mathrm{~Hz})$. Incidentally, one of the advantages of a monochromatic experiment is that the frequency dispersion law of the intrinsic attenuation, which is difficult to establish experimentally, particularly at low frequencies $(<1 \mathrm{kHz})$, does not enter into consideration.

\subsection{Noise}

As demonstrated in Chapter $V$, the Gelfand-Levitan algorithm is stable, with small errors in the reflection coefficient resulting in small errors in the reconstructed velocity. The numerical experiments we have conducted by adding zero-mean Gaussian stationary noise to the reflection coefficient input support our previous conclusion.

Gaussian noise was added to both the real and imaginary part of the reflection coefficient generated by the method of Section 6.1 . The resulting reflection coefficient was submitted to the usual steps involved in the inversion procedure, namely the Fourier transform step followed by the application of the Gelfand-Levitan algorithm. 
The signal to noise ratio (SNR) is defined as

$$
\mathrm{SNR}=10 \log _{10}\left(\frac{I_{s}}{I_{n}}\right)
$$

where the signal and noise powers for $\mathrm{N}$ discrete points are

$$
I_{s}=\frac{1}{N} \sum_{n=1}^{N}\left|r_{s}\right|^{2}
$$

and

$$
I_{n}=\sigma_{n}^{2} \text {, the variance of the noise. }
$$

As shown in Fig. 31 for a window profile, the perturbation of the reflection coefficient by the addition of zeromean Gaussian noise $(\sigma=0.1)$ results in a roughly proportional degradation of the reconstructed potential.

The preliminary assessment of the effect of noise leads us to conclude that the Gelfand-Levitan inverse method is stable in the presence of noise. This analysis can be refined in the future by including a more pertinent model for the noise based on Mook's results ${ }^{(64)}$; Mook has shown that the addition of zero-mean stationary white Gaussian noise to the point source pressure field resulted in a non-stationary variance with the noise power concentrated near the vertical $\left(\mathrm{k}_{\mathrm{z}}=\mathrm{k}_{0}\right.$, the water wavenumber $)$. One should also note that the measured reflection coefficient itself can be regarded 
as a non-stationary process with a mean that varies between one near grazing incidence and zero at infinity. That consideration may lead to a more appropriate measure of performance than that of SNR presented here. 


\section{CONCLUSION}

In this thesis we have examined the theoretical and computational underpinnings of a novel approach to the determination of the acoustic parameters of the oceanic sediment layer. Traditional marine seismic methods, acoustic reflection and refraction measurements, "yield no velocity information in the top of the first sediment layer which is of critical interest for modeling the sea floor for underwater acoustics" (34). That is precisely where the method we have analyzed in the thesis is most accurate. Interval velocity calculations are restricted to layer thicknesses larger than one twelfth the water depth (34), and subsequently the velocity at the sediment interface cannot be determined accurately. In the absence of in situ core measurements of surface velocity $v_{0}$, the velocity gradient at the top of the sediment column is also uncertain. The direct inverse method presented here requires only the a priori knowledge of the speed of sound in the water above the sediment layer.

By operating at a single frequency, the effects of dispersion are separated from the propagation process. Moreover, the dispersive characteristics of the medium can be studied by performing the experiment at various frequencies. 
A single frequency, steady-state experiment also means that the measurement relies on amplitudes rather than on arrival times (explosive methods). While it is true that time is measured more accurately than acoustic pressure, arrival times can not always be interpreted correctly due to multiple reflections. Amplitude, in turn, may be affected by a host of factors to which arrival time is insensitive such as loss and diffraction; it is not yet possible to judge the relative merits of the two methods in the absence of appropriate experitmental data.

\section{Contributions}

The determination of the acoustic properties of the ocean bottom was shown to be equivalent to the reconstruction of an unknown potential in a Schrodinger equation from the plane-wave reflection coefficient given at all angles of incidence (Chapter II).

The pivotal role of the reflection coefficient lead us to a detailed examination of its properties in Chapter III. In particular, we showed, by induction, that the reflection coefficient decays at least as rapidly as $\left(1 / \mathrm{k}_{\mathrm{z}}{ }^{2}\right)$ and is therefore integrable.

The derivation of our approach to the direct inversion method was presented in Chapter IV. The Gelfand-Levitan method was extended to the case where the acoustic velocities on either side of a slab are different. That is, of course, the case in the ocean bottom problem where the acoustic velocity 
in the basement is larger than the acoustic velocity in the water. We also showed that the neglect of bound states is justified at the current operating frequency in both clayey silt and in silty clay.

Three methods for the numerical solution of the Gelfand-Levitan integral equation were investigated (Chapter V). The first method we developed is a series expansion of the solution obtained by successive approximations. The first two terms of this expansion represent a substantial improvement over the well known Born approximation. The other two numerical methods presented in Chapter $\mathrm{V}$ are based on the discretization of the Gelfand-Levitan integral equation. They represent two ways to bypass the matrix inversion inherent in a straightforward solution of the discretized equation. We then obtained estimates for the bound on the error in the integral of the potential due to discretization errors and due to errors in the reflection coefficient.

In Chapter VI, we discussed the numerical results obtained from the inversion of synthetic data. By dealing with synthetic data, we insured that the bottom profile was known exactly and that the effectiveness of the method could be studied without any fear of experimental imperfections. We concluded that the Gelfand-Levitan method appears to be very accurate at the top of a sediment column, just where the more usual methods are least accurate. The resolution 
obtained is less than the wavelength of the acoustic source in the water. The degradation of the reconstructed velocity profile becomes, however, pronounced if the reflection data is restricted to real angles above critical. Perturbations of data were also studied. Perturbations such as intrinsic loss in the acoustic medidm $c_{\perp}$ noise in the data produce perturbations in the reconstructed profile. The inclusion of density variations requires the use of two frequencies and two separate inversions of the Gelfand-Levitan equation. We were able to gauge the performance of the numerical schemes through the study of the inversion of the acoustic profile for two half-spaces (constant velocity), for which we derived the Fourier transform of the reflection coefficient analytically. The improvements wrought by the improved Born method are clear, as are the effects of sampling on the reconstructed profile. The improved Born method represents a fast and easy to implement method of recovering the velocity at the top of the sediment column. The two finite difference methods are more time-consuming but yield an accurate reconstruction of the acoustic profile over greater depth.

Future Work

On the theoretical front, we suggest a thorough investigation of the Gelfand-Levitan method in the presence 
of density variations. It may also be useful to incorporate loss directly into the original formulation. One should also seek efficient numerical implementations of the GelfandLevitan algorithm that could increase the penetration depth of the reconstruction. In this respect, we think that the combination of the Gelfand-Levitan algorithm with a priori information such as the acoustic properties of the basement might constitute a promising approach.

On the experimental front, the testing of the GelfandLevitan inverse method on field data should be given priority to determine its ultimate value. If an actual ocean-based experiment were precluded at the moment, we would suggest carrying out a similar electromagnetic experiment on dielectrics at microwave frequencies.

On the numerical front, one would want to test the whole experimental scheme, starting from pressure measurements due to a point source and ending with the Gelfand-Levitan inversion. Special attention should be paid to the effect of noise in the recording of pressure on the plane-wave reflection coefficient and ultimately on the reconstructed acoustic parameters. 
It is remarkable that the Frisk experiment which was conceived on purely intuitive grounds, when modelled theoretically, bears out the expectation that accurate results are achievable. To date, experimental data has been interpreted by time - consuming trial and error procedures. Our mathematical and numerical approach suggests that a more direct inverse method for processing experimental data, requiring no a priori information about the acoustic parameters of the bottom is feasible. 


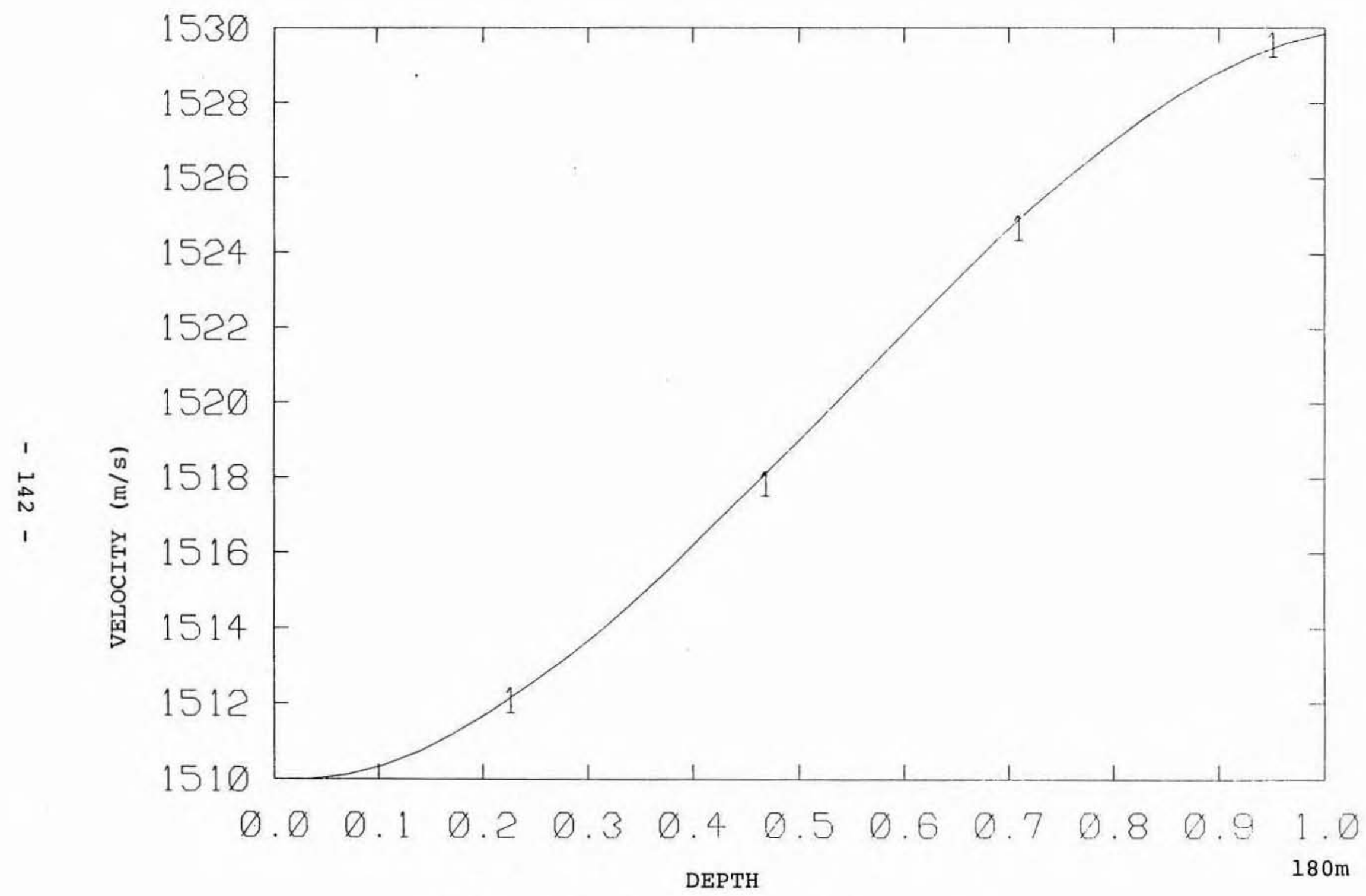

Figure 10: Stickler Profile
\[ \mathrm{C}_{0}=1510 \mathrm{~m} / \mathrm{s}, \mathrm{c}_{1}=1530 \mathrm{~m} / \mathrm{s}, \mathrm{L}=180 \mathrm{~m} \] 


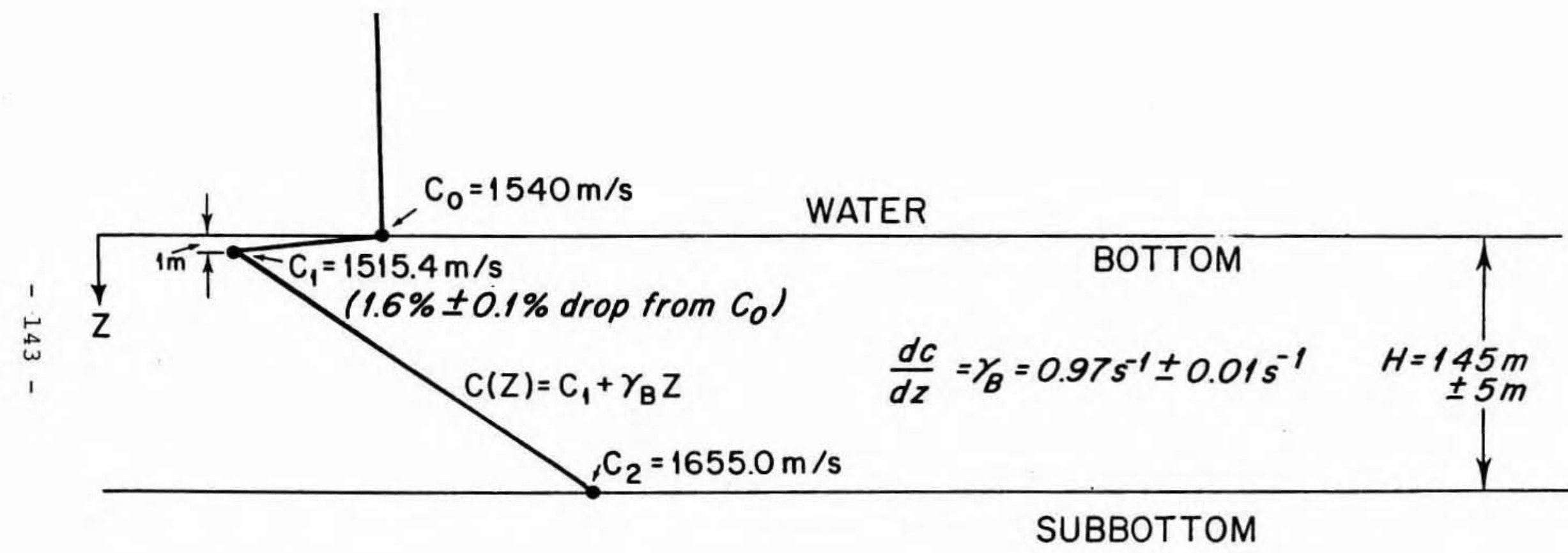

Figure 11: Frisk Profile 


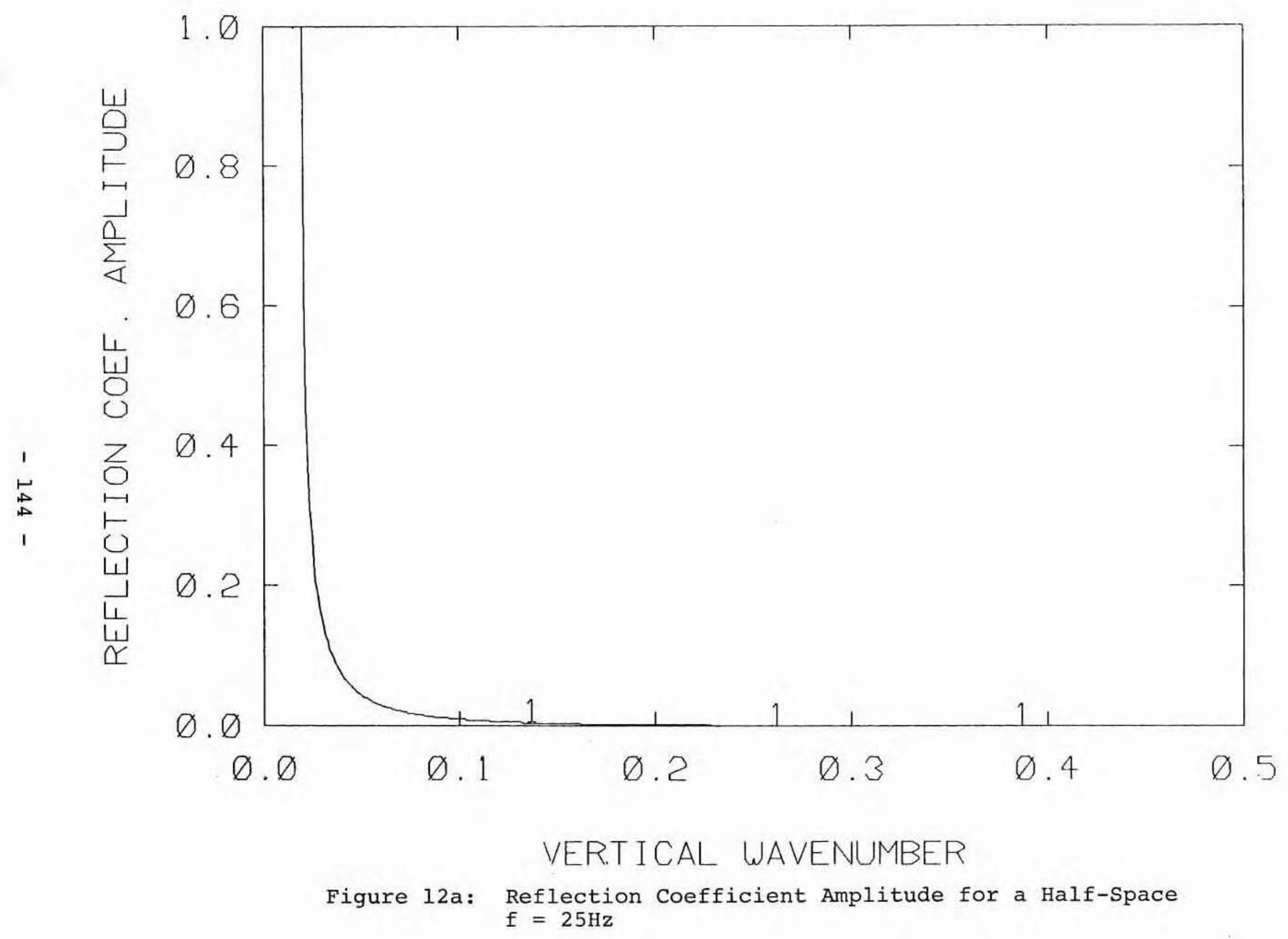




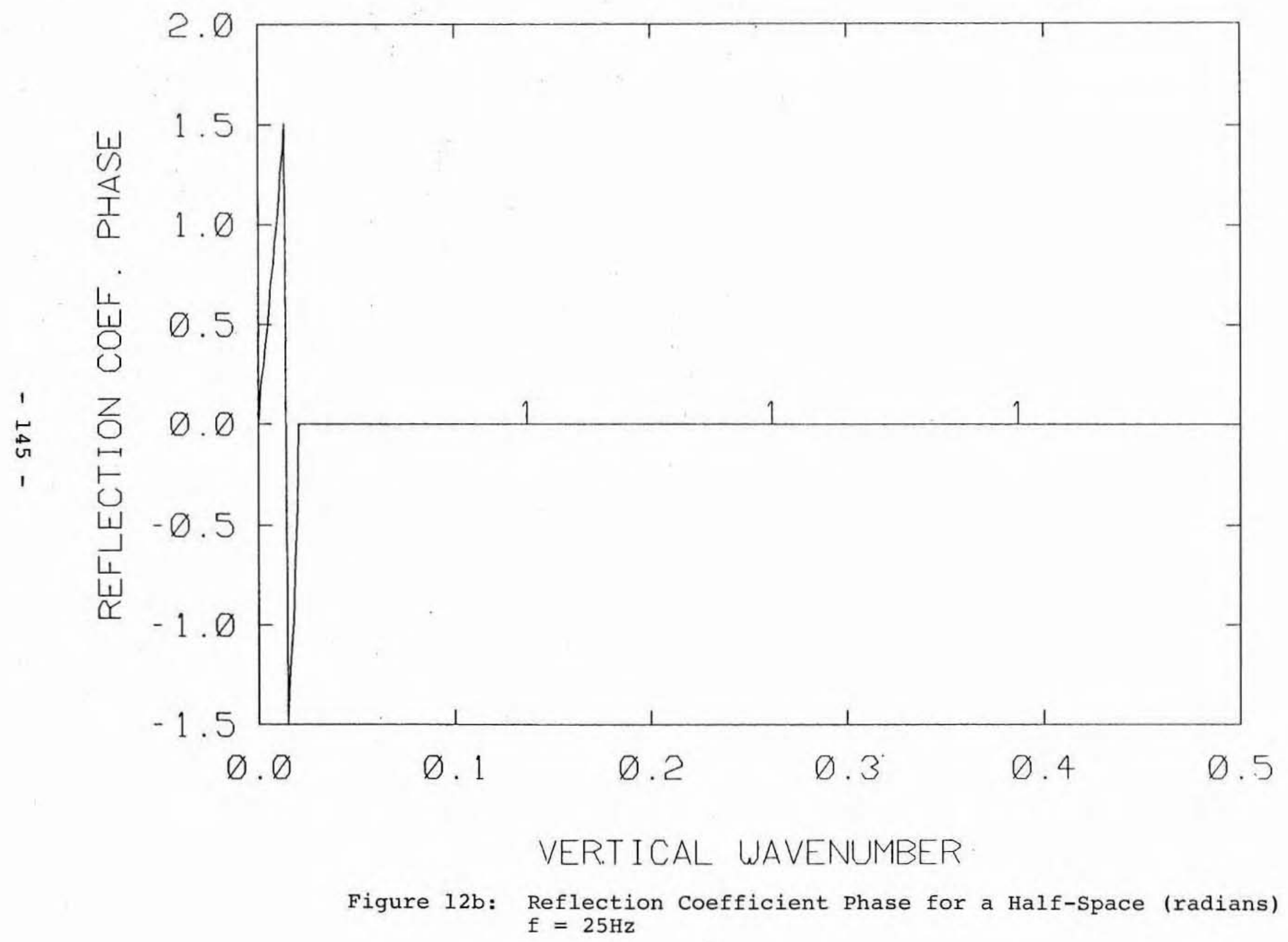




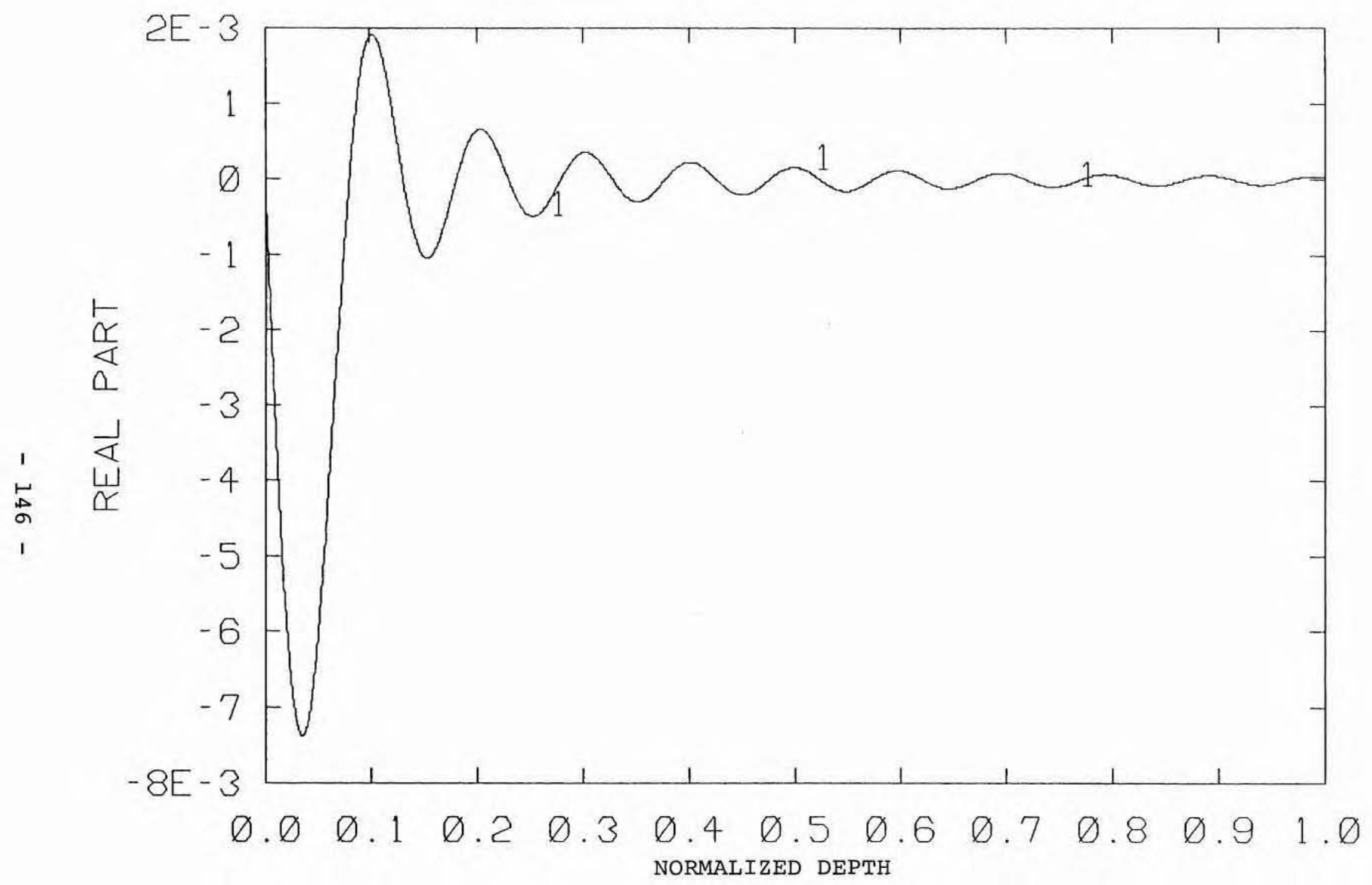

Figure 12c: Fourier Transform of Figure $12(\mathrm{a}, \mathrm{b})$. 


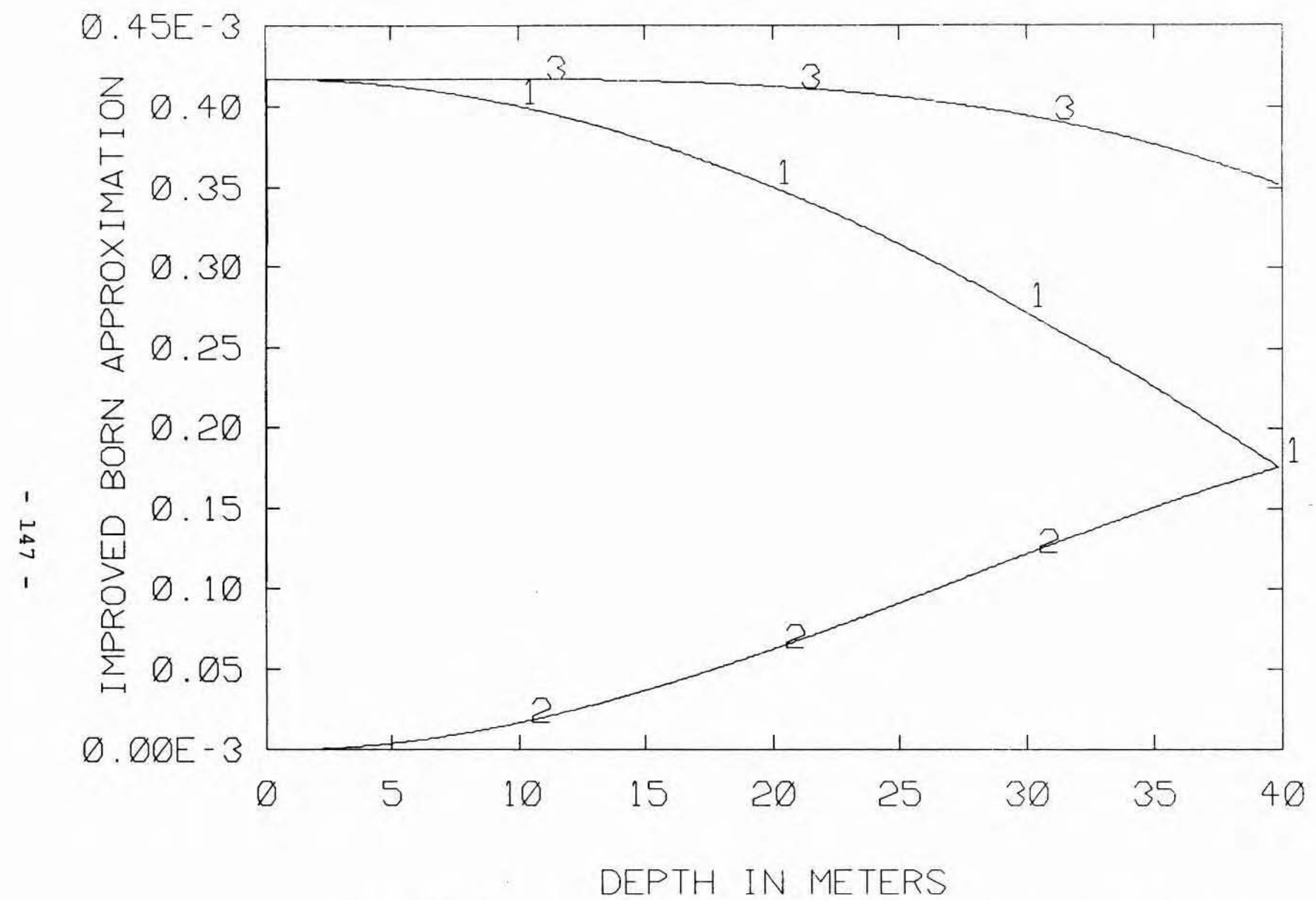

Figure 12d: Half-Space Potential Reconstruction, $c_{0}=1510 \mathrm{~m} / \mathrm{s}$, $c_{1}=1540 \mathrm{~m} / \mathrm{s}, 1:$ Born, 2: Correction, $3:$ Improved Born $=(1)+(2)$ $\mathrm{f}=25 \mathrm{~Hz}$ 


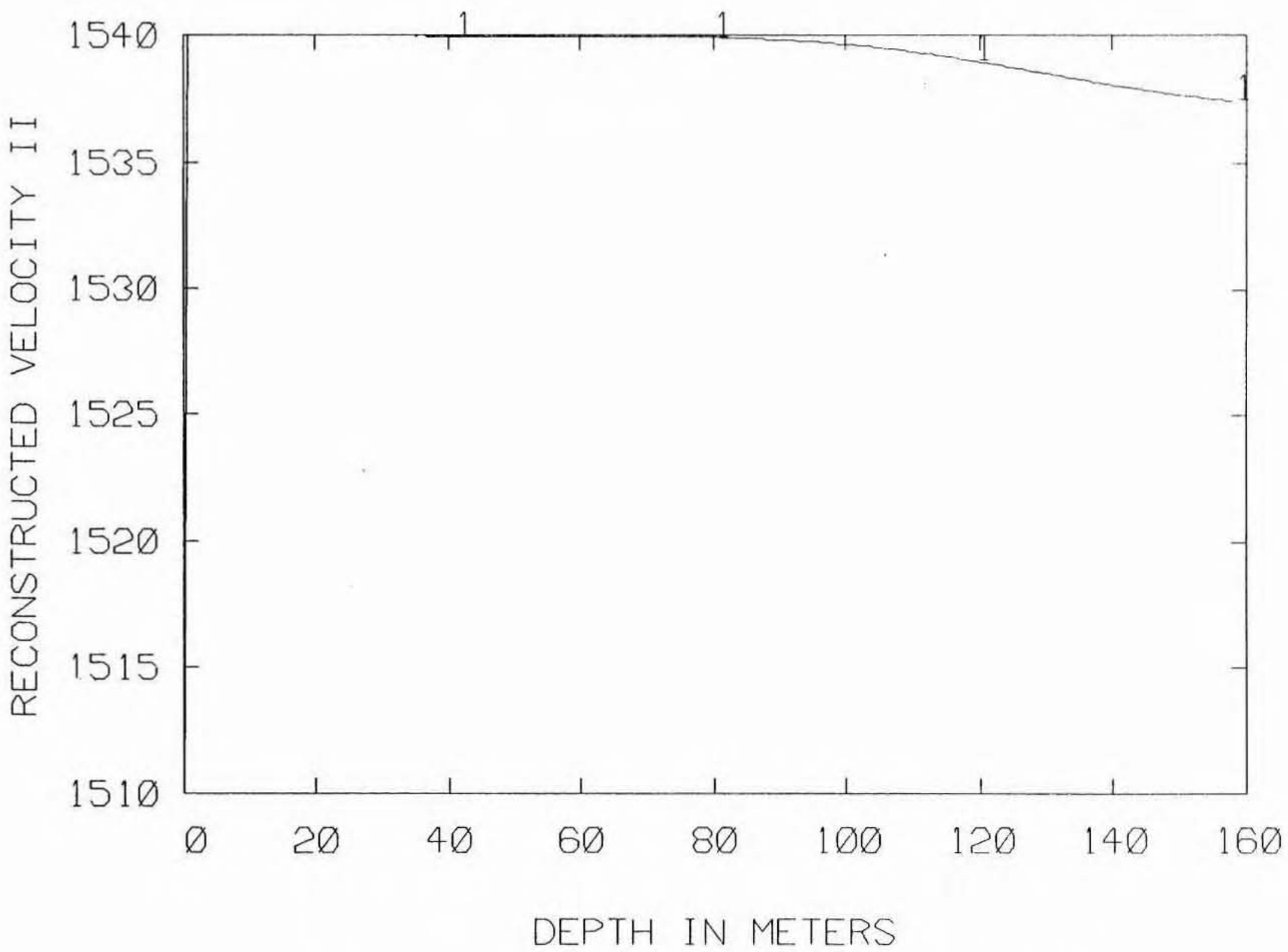

Figure 13: Velocity Reconstruction for a Half-Space (m/s)

$R(z)$ obtained from analytic expression $-\Delta z=0.767 \mathrm{~m}$ 


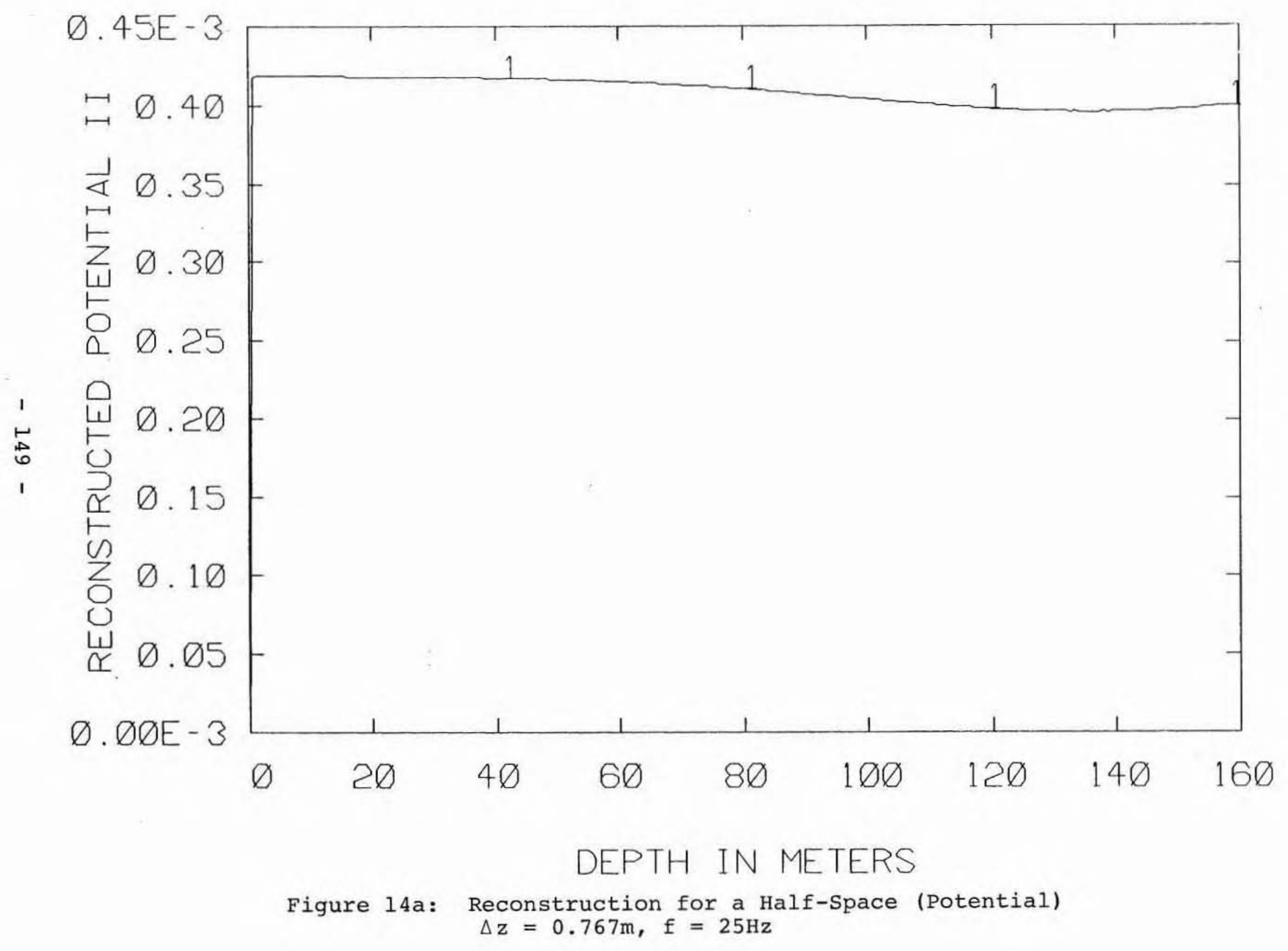




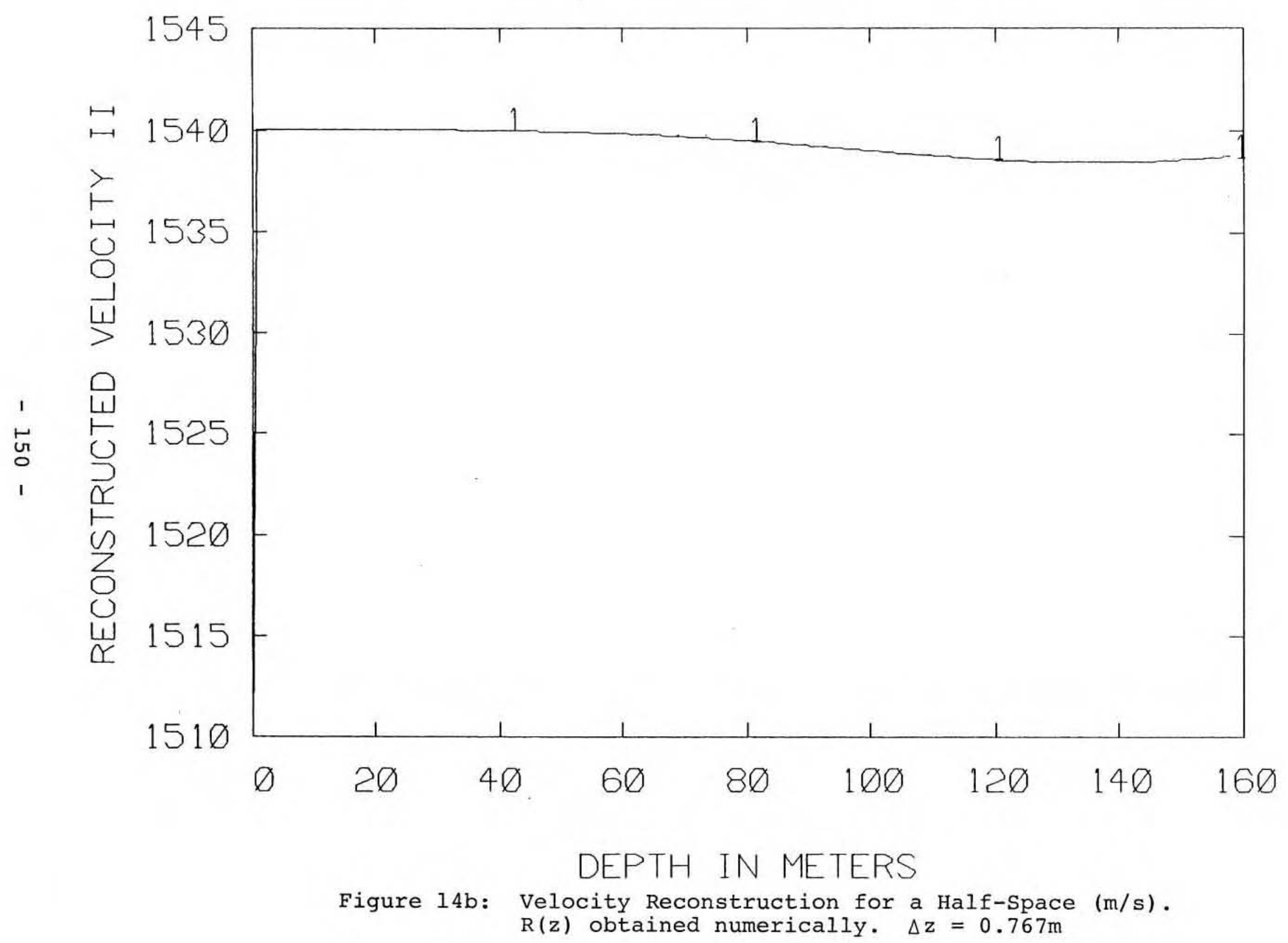




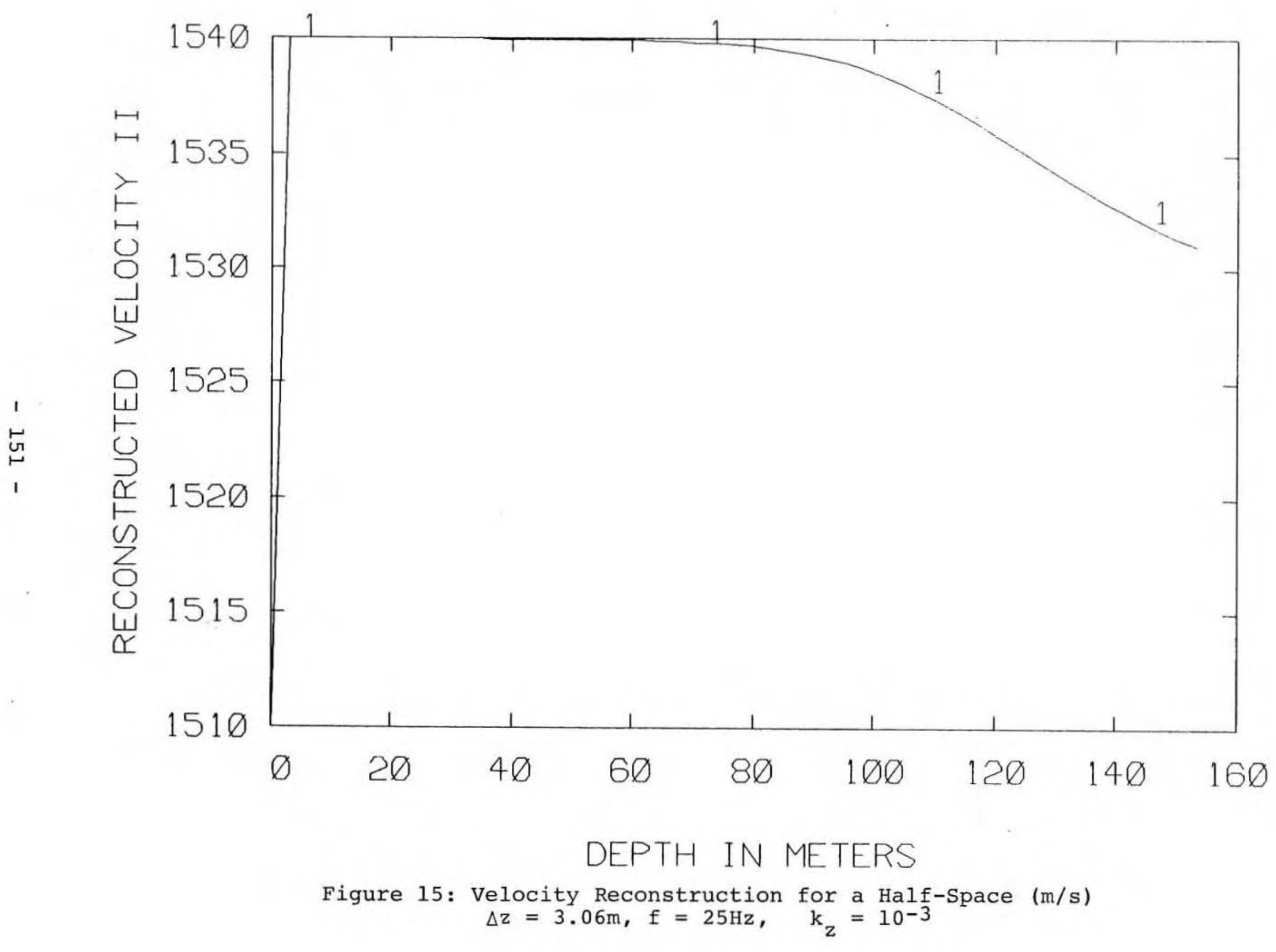




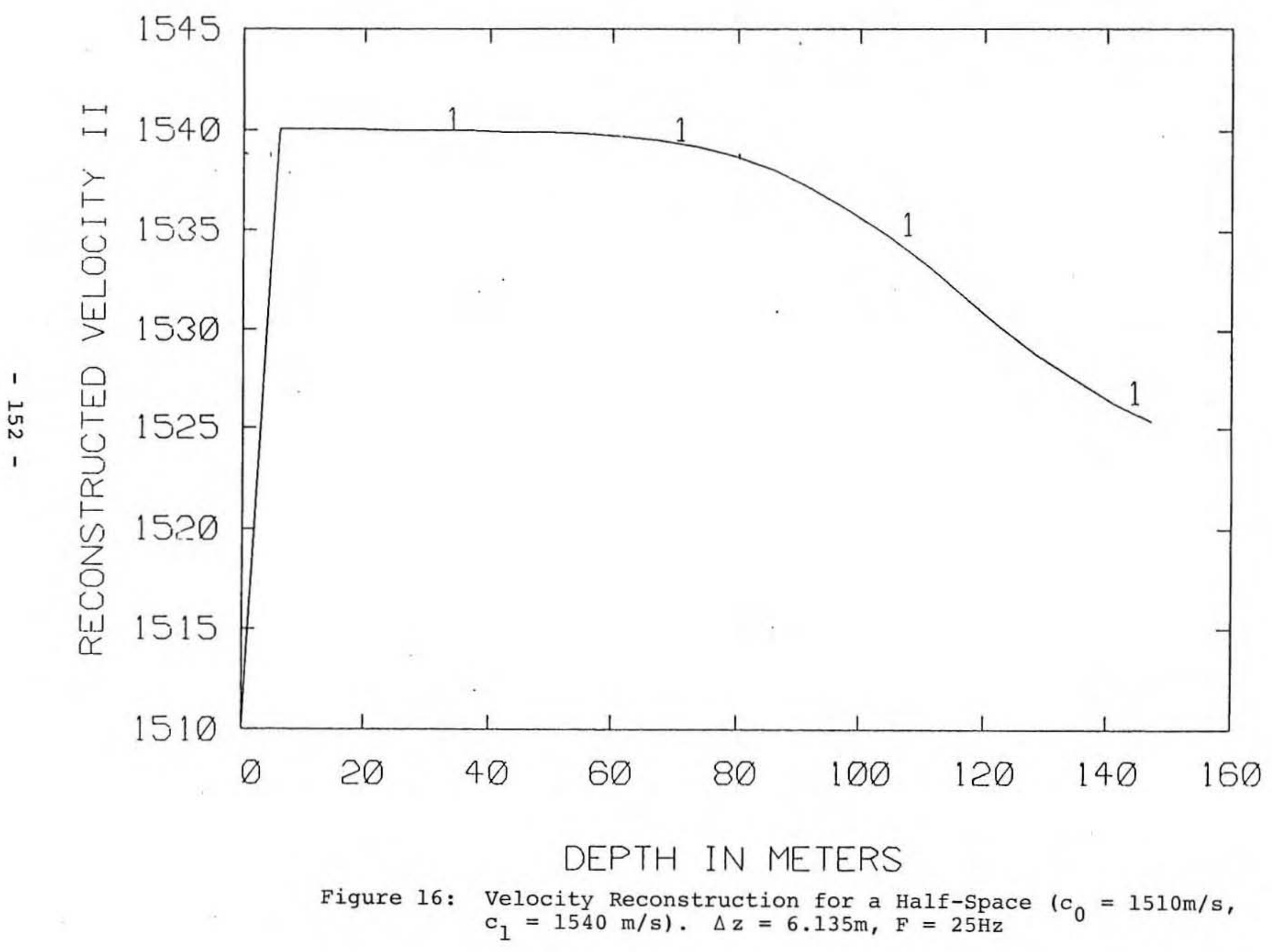




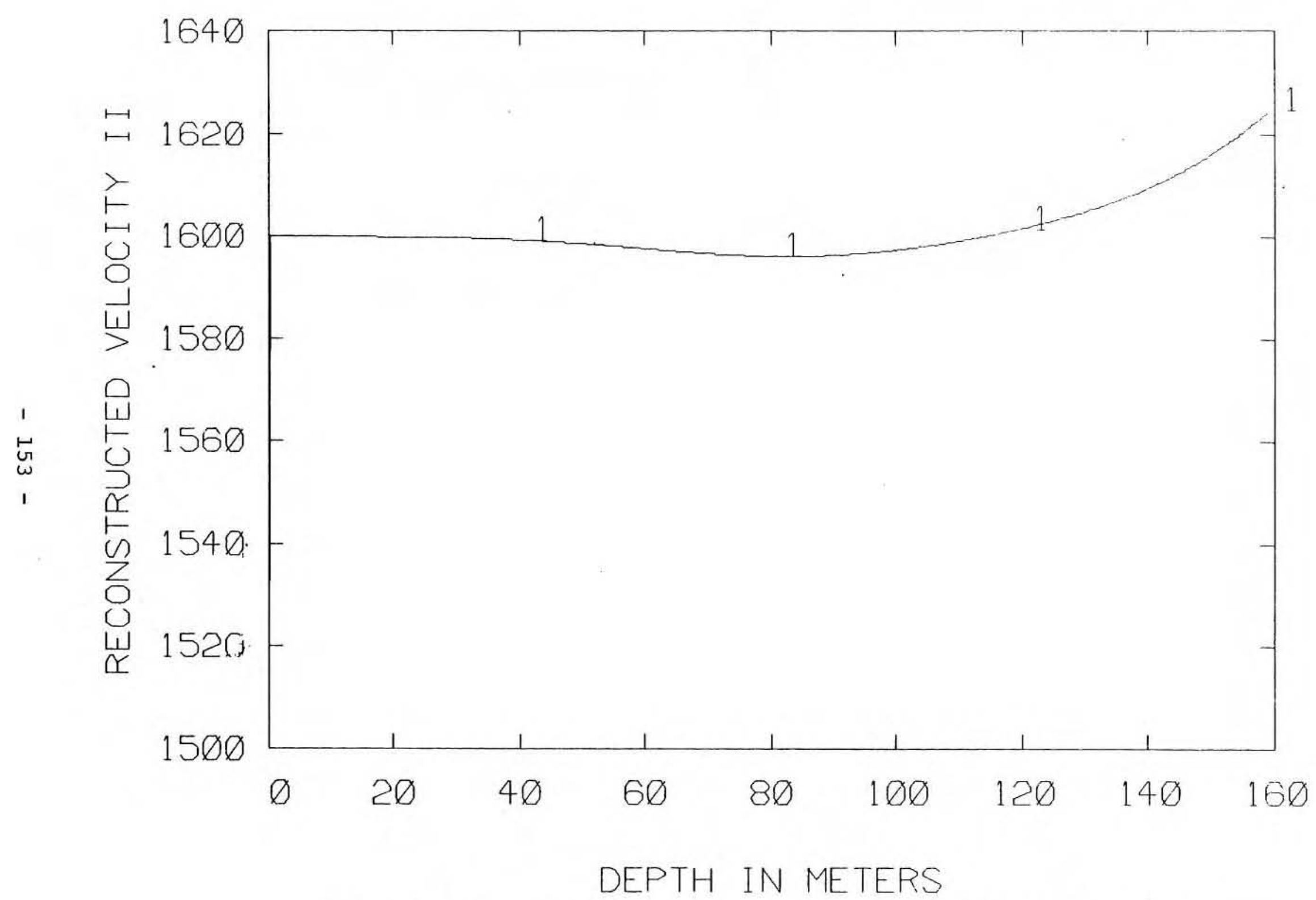

Figure 17a: Velocity Reconstruction for a Half-Space. $\left.\mathrm{c}_{0}=1500 \mathrm{~m} / \mathrm{s}, \mathrm{c}_{1}=1600 \mathrm{~m} / \mathrm{s}\right) \cdot \Delta \mathrm{z}=0.3125 \mathrm{~m}, \mathrm{f}=25 \mathrm{~Hz}$ 


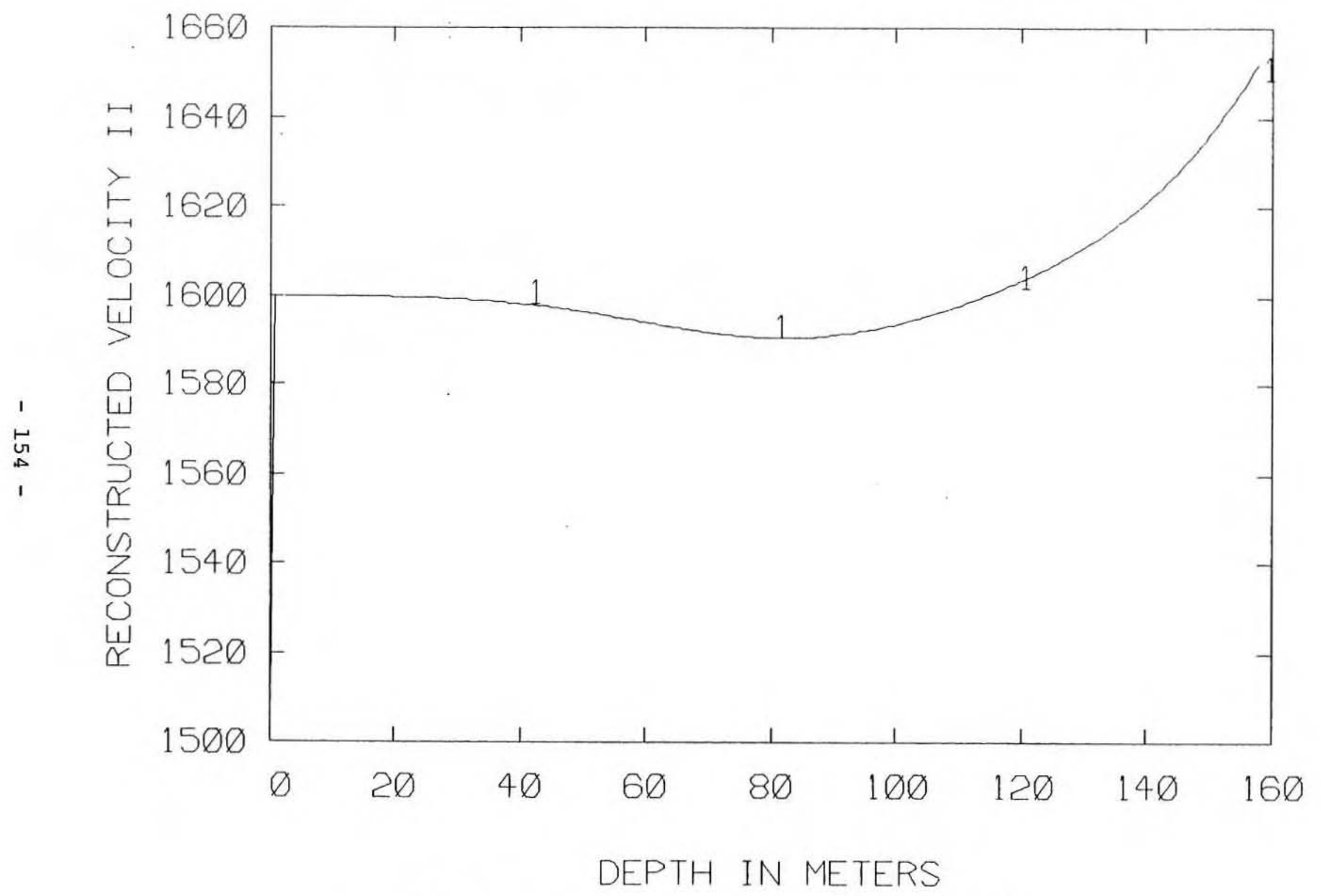

Figure 17b: Velocity Reconstruction for Half-Space.

$\left(c_{0}=1500 \mathrm{~m} / \mathrm{s}, c_{1}=1600 \mathrm{~m} / \mathrm{s}\right)$.

$\Delta \mathrm{z}=0.767 \mathrm{~m}, \mathrm{f}=25 \mathrm{~Hz}$. 


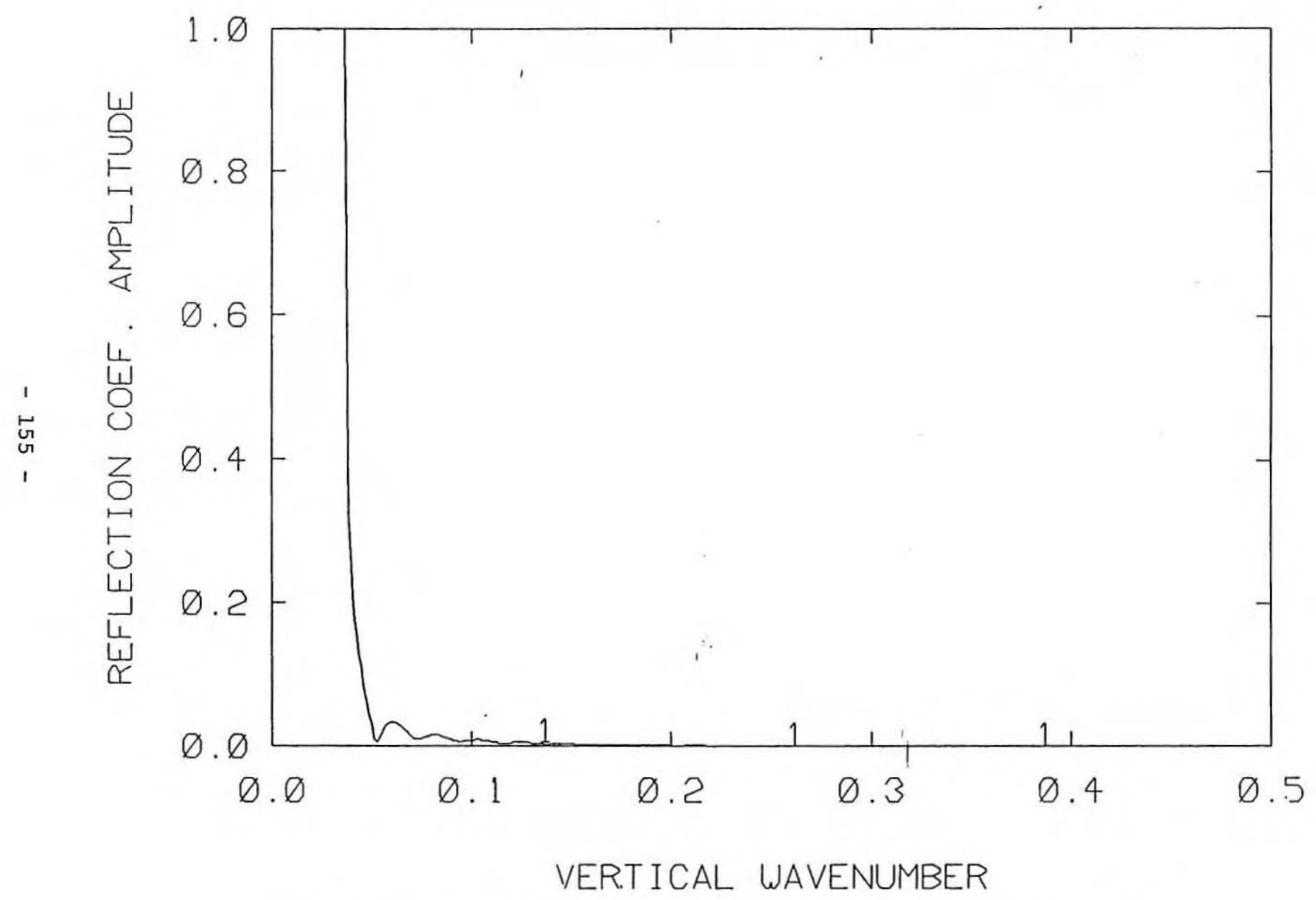

Figure 18a: Reflection Coefficient Amplitude. Frisk Profile $\mathrm{k}_{0}=0.1, \mathrm{f}=25 \mathrm{~Hz}$. 


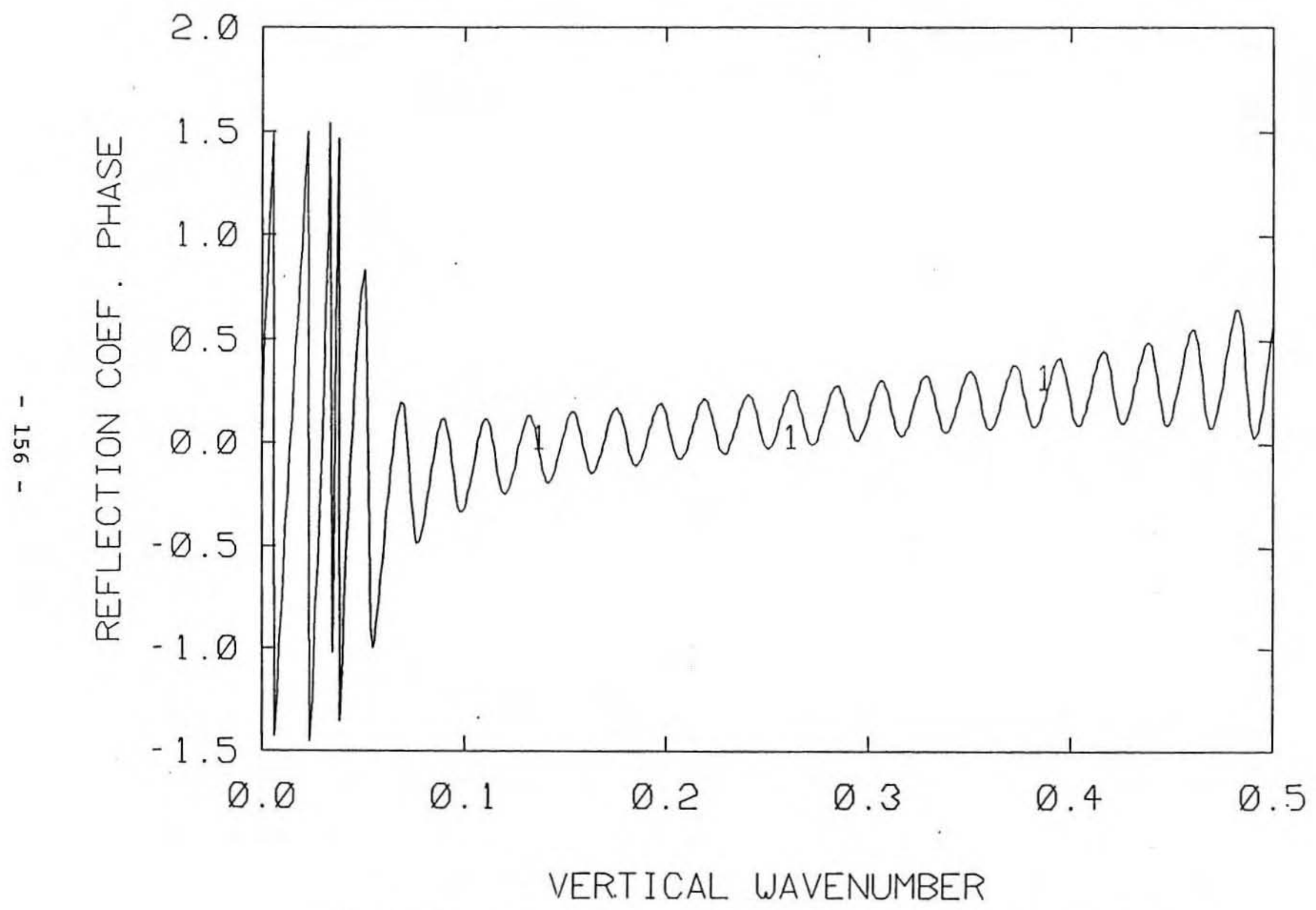

Figure 18b: Reflection Coefficient Phase - Frisk Profile. $f=25 \mathrm{~Hz}$ 


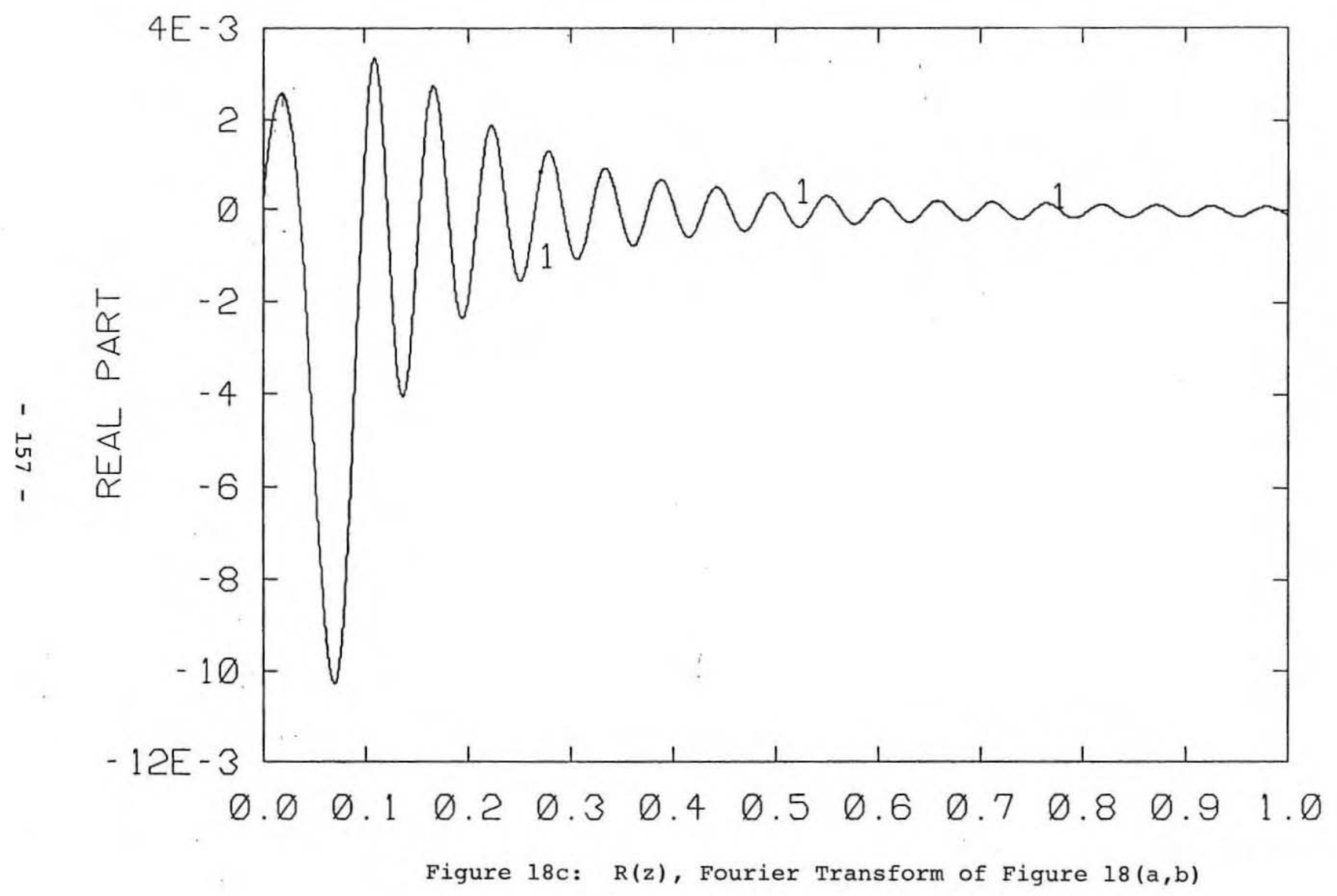




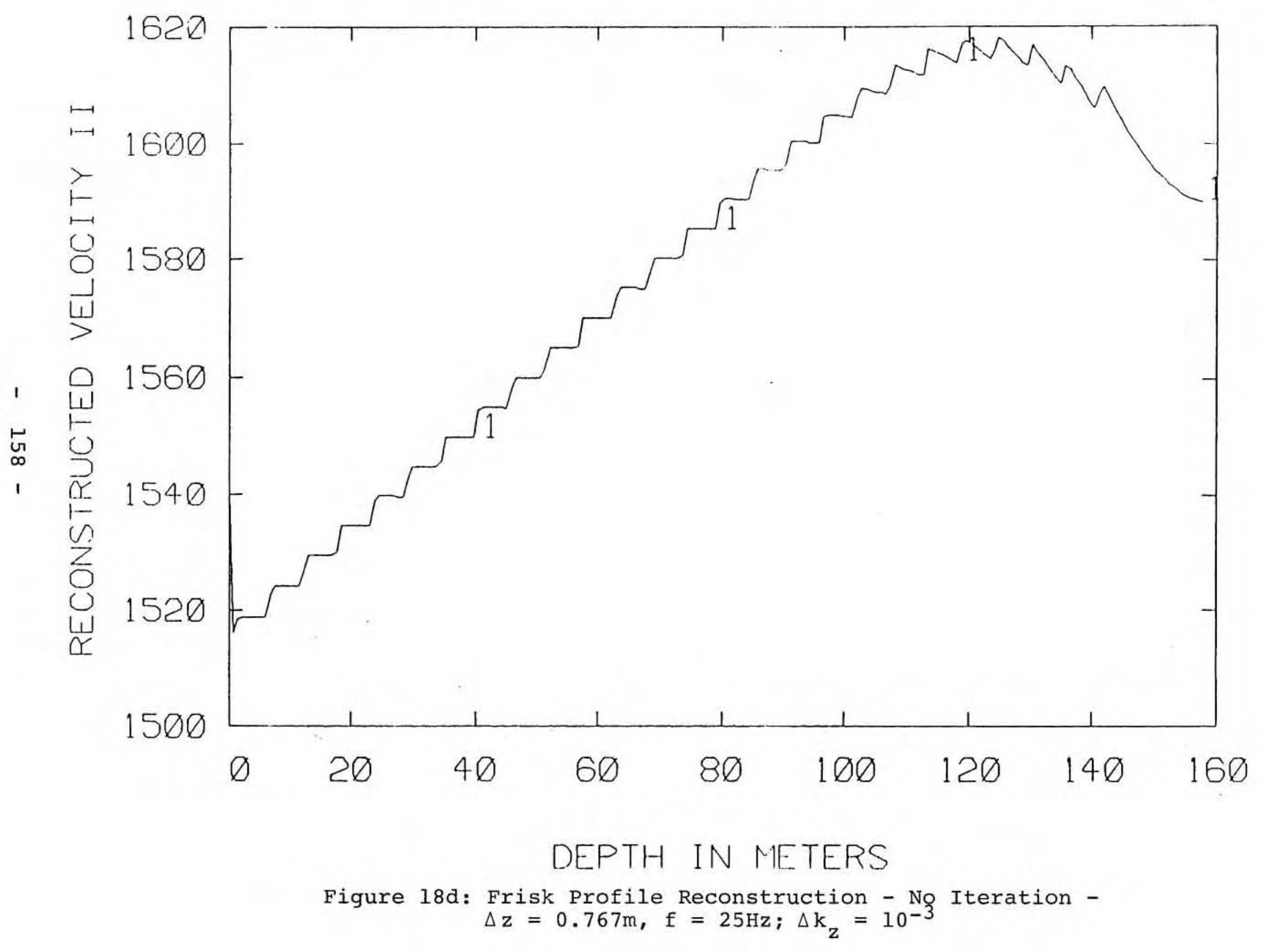




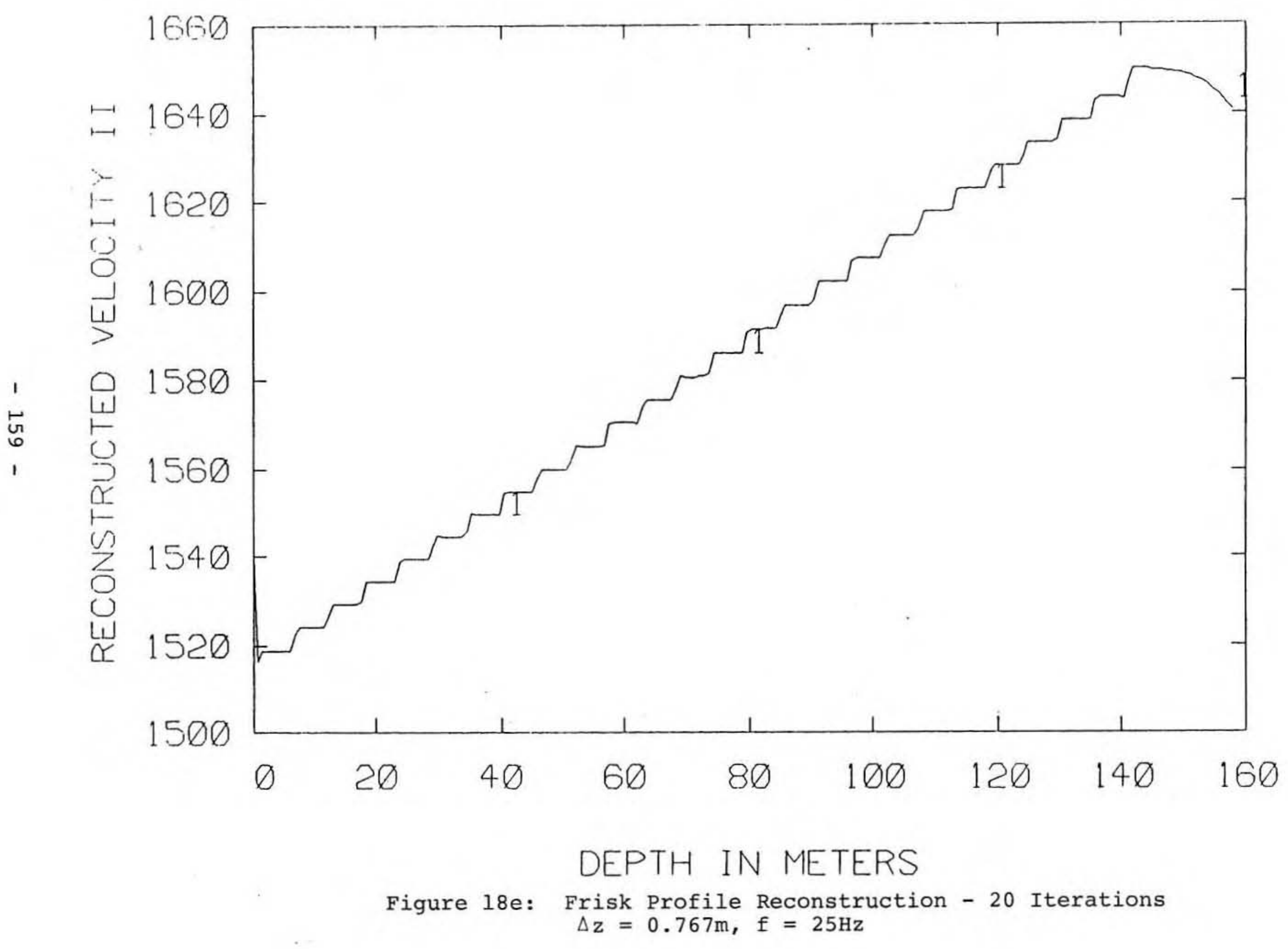




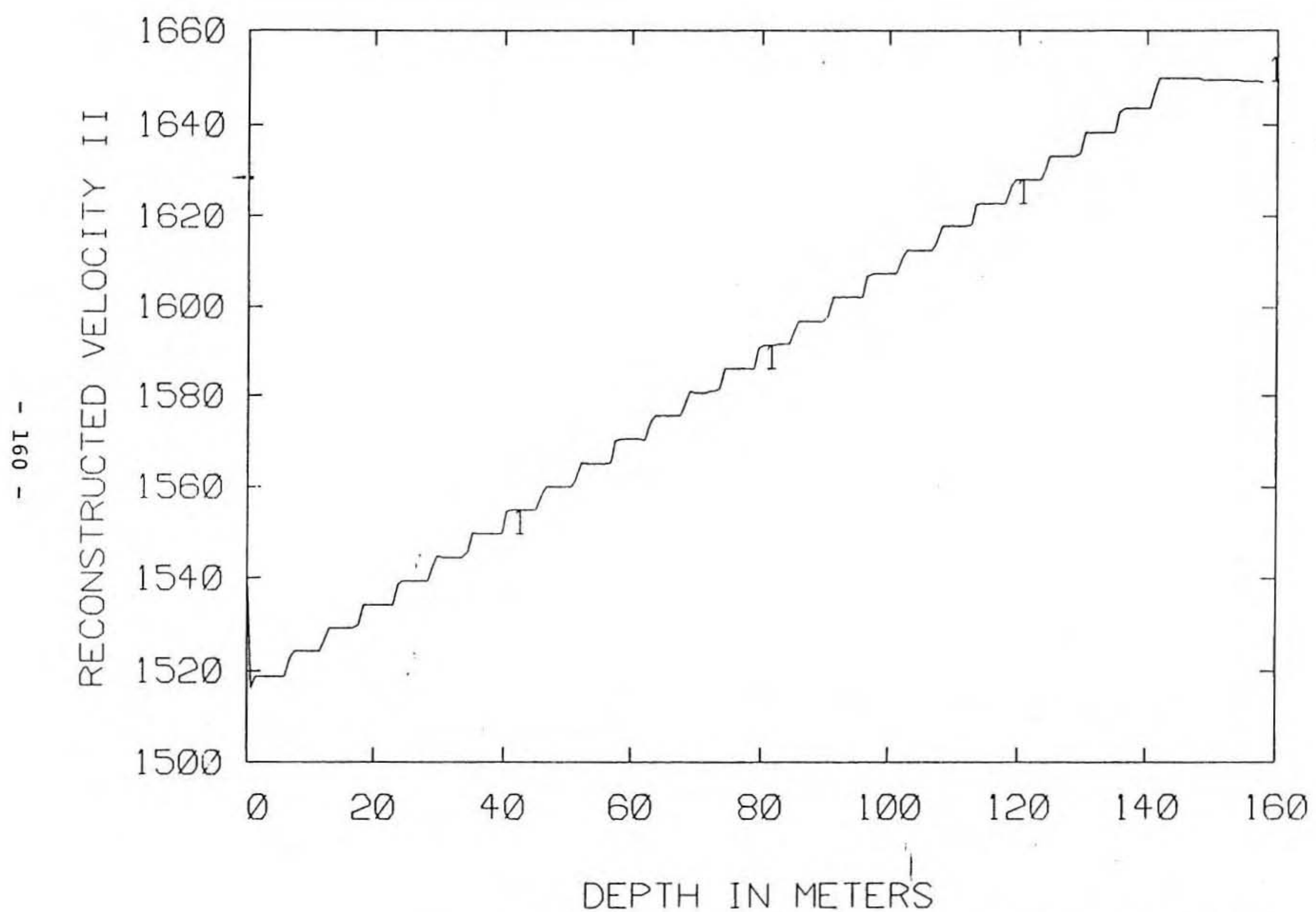

Figure 18f: Frisk Profile Reconstruction - 40 Iterations $\Delta z=0.767 \mathrm{~m}, f=25 \mathrm{~Hz}$ 


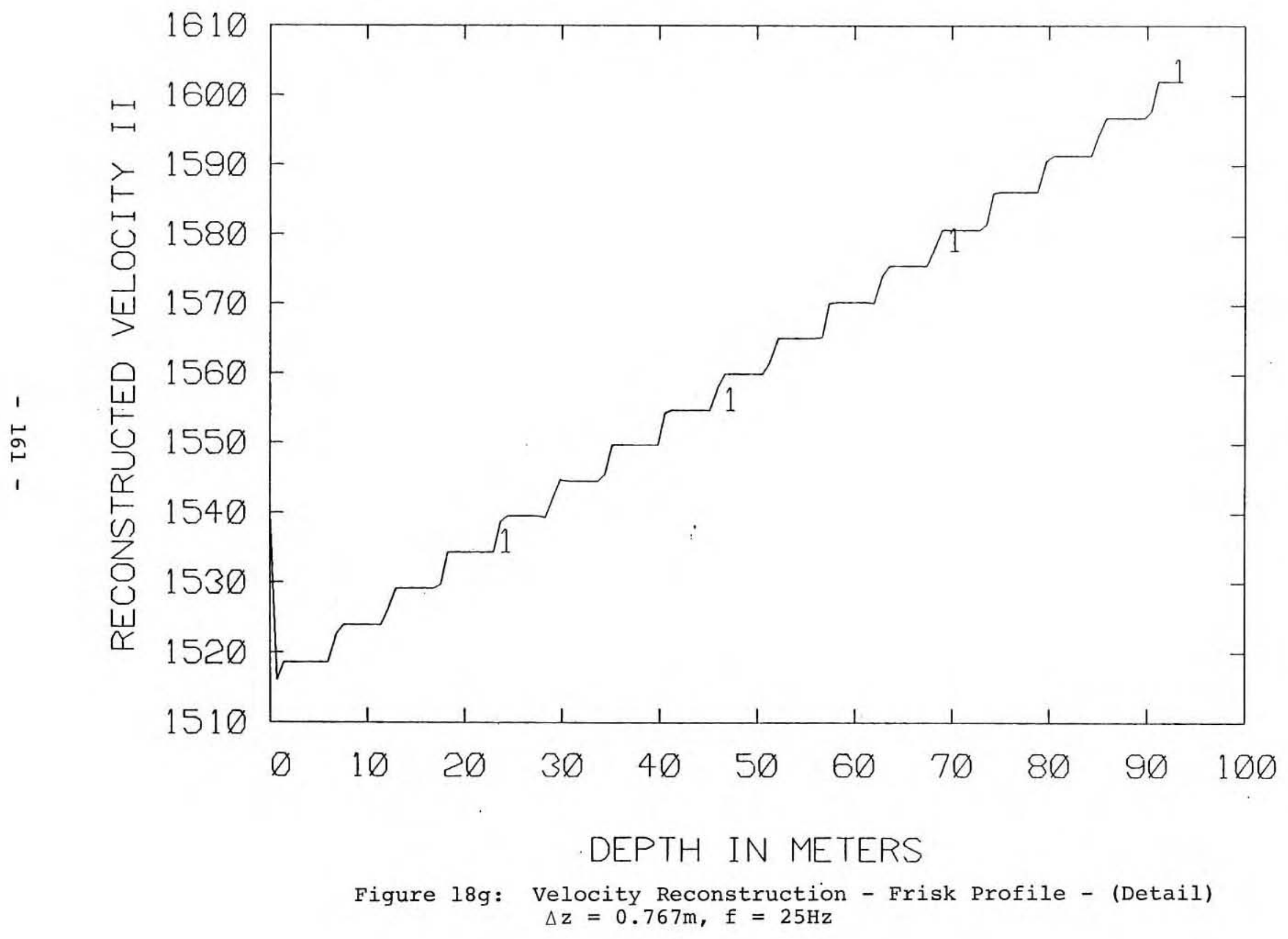




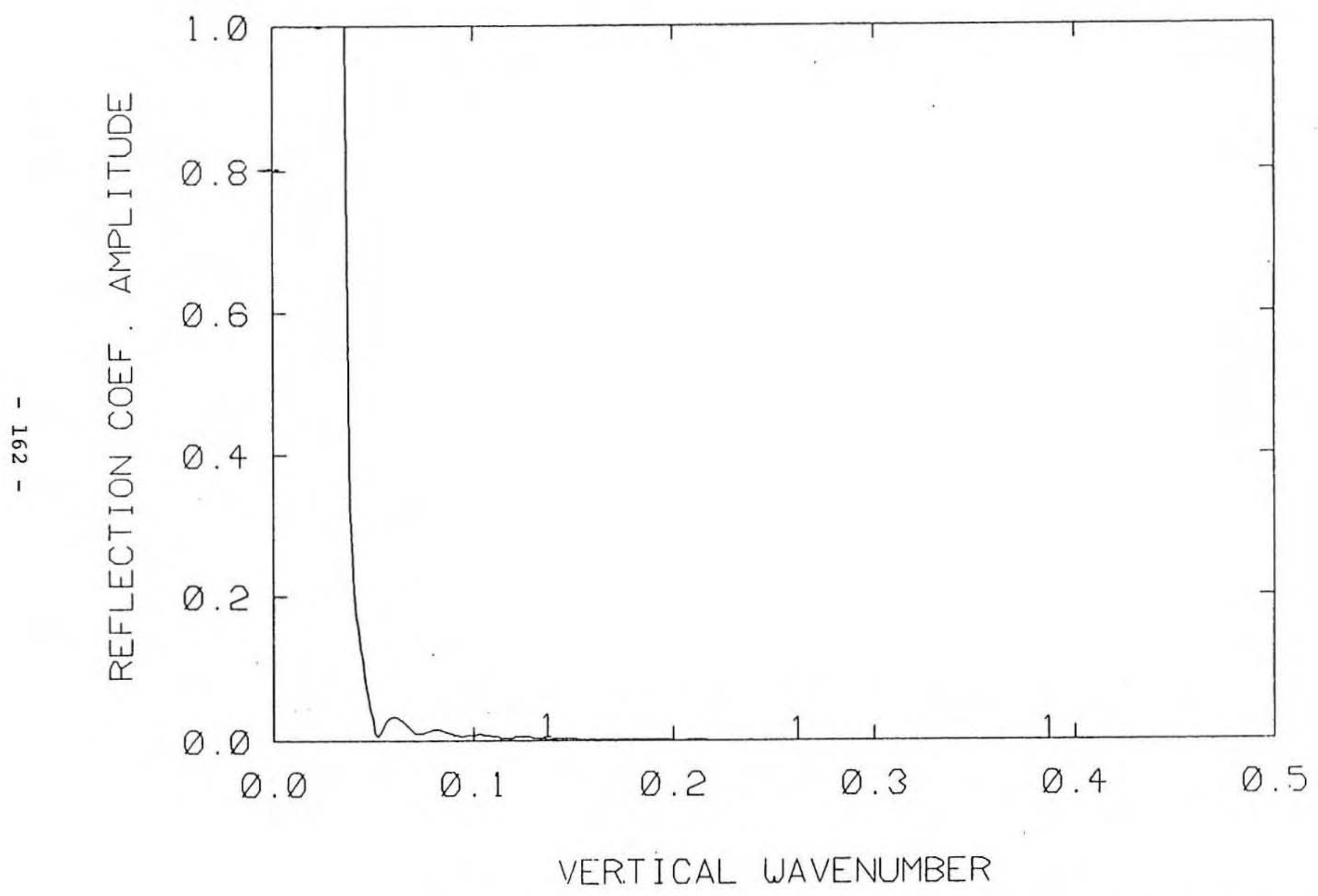

Figure 19a: Reflection Coefficient Amplitude. Frisk Profile Limited Range, $\mathrm{K}_{\mathrm{zmax}}=0.512, \mathrm{f}=25 \mathrm{~Hz}$ 


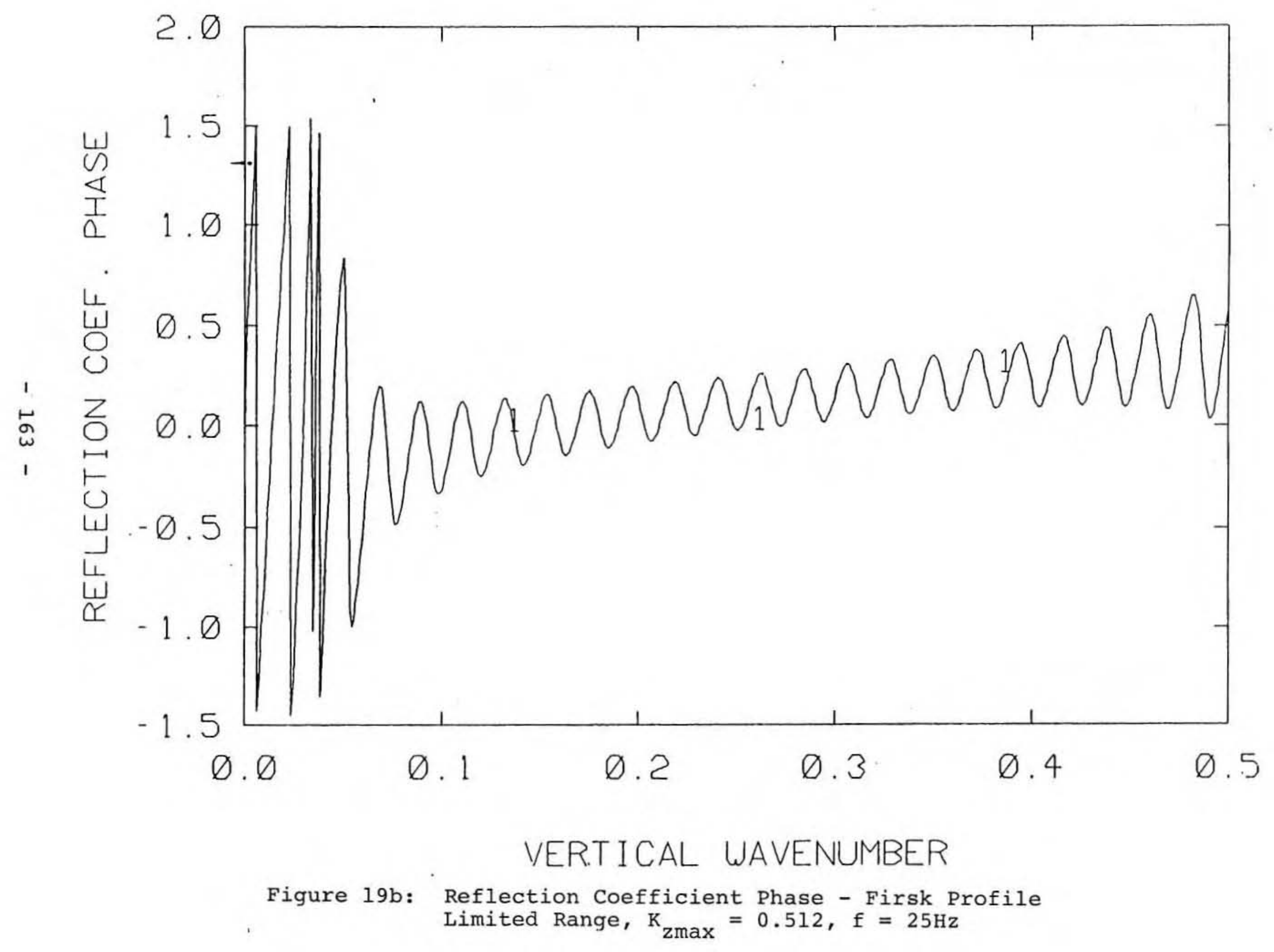




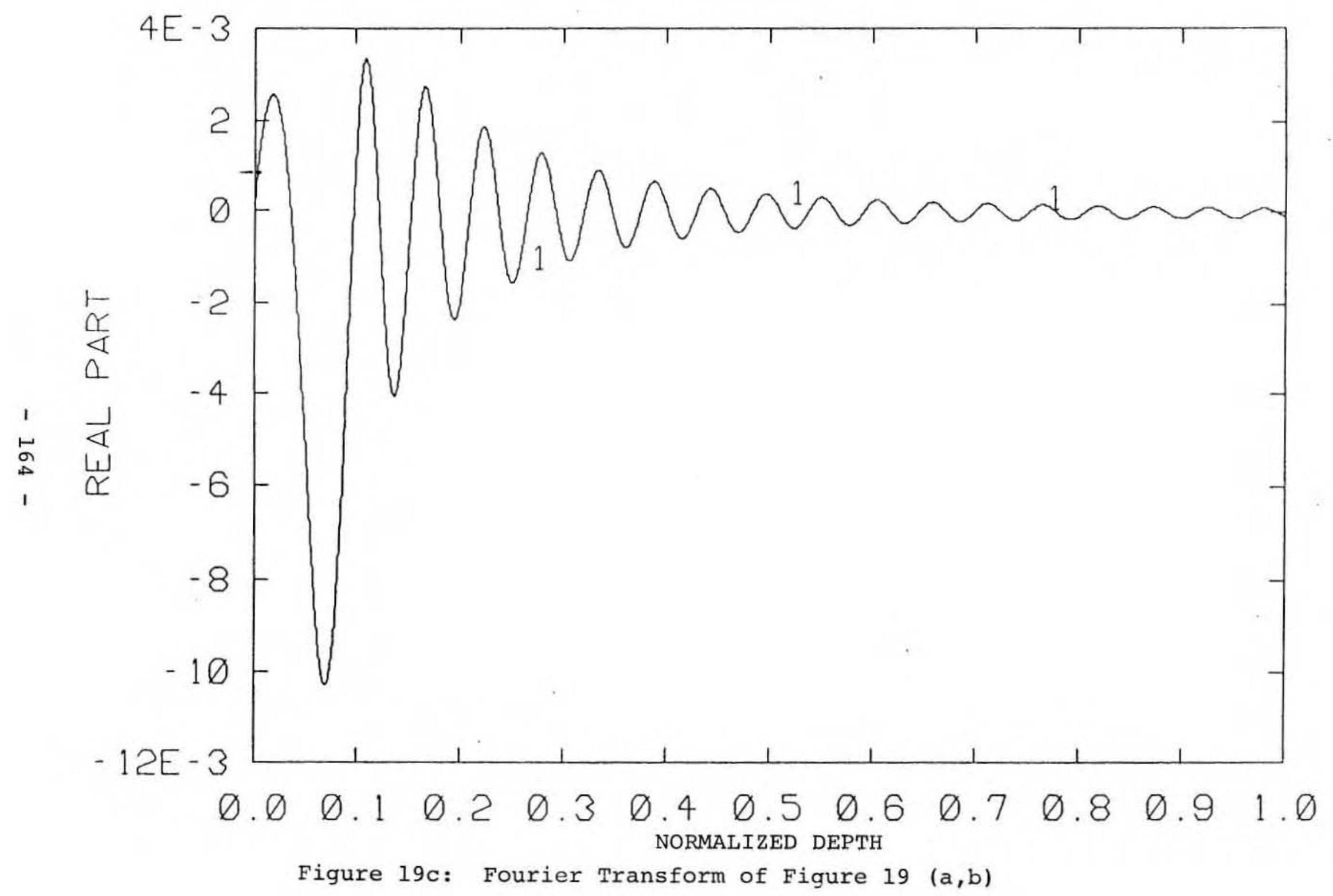




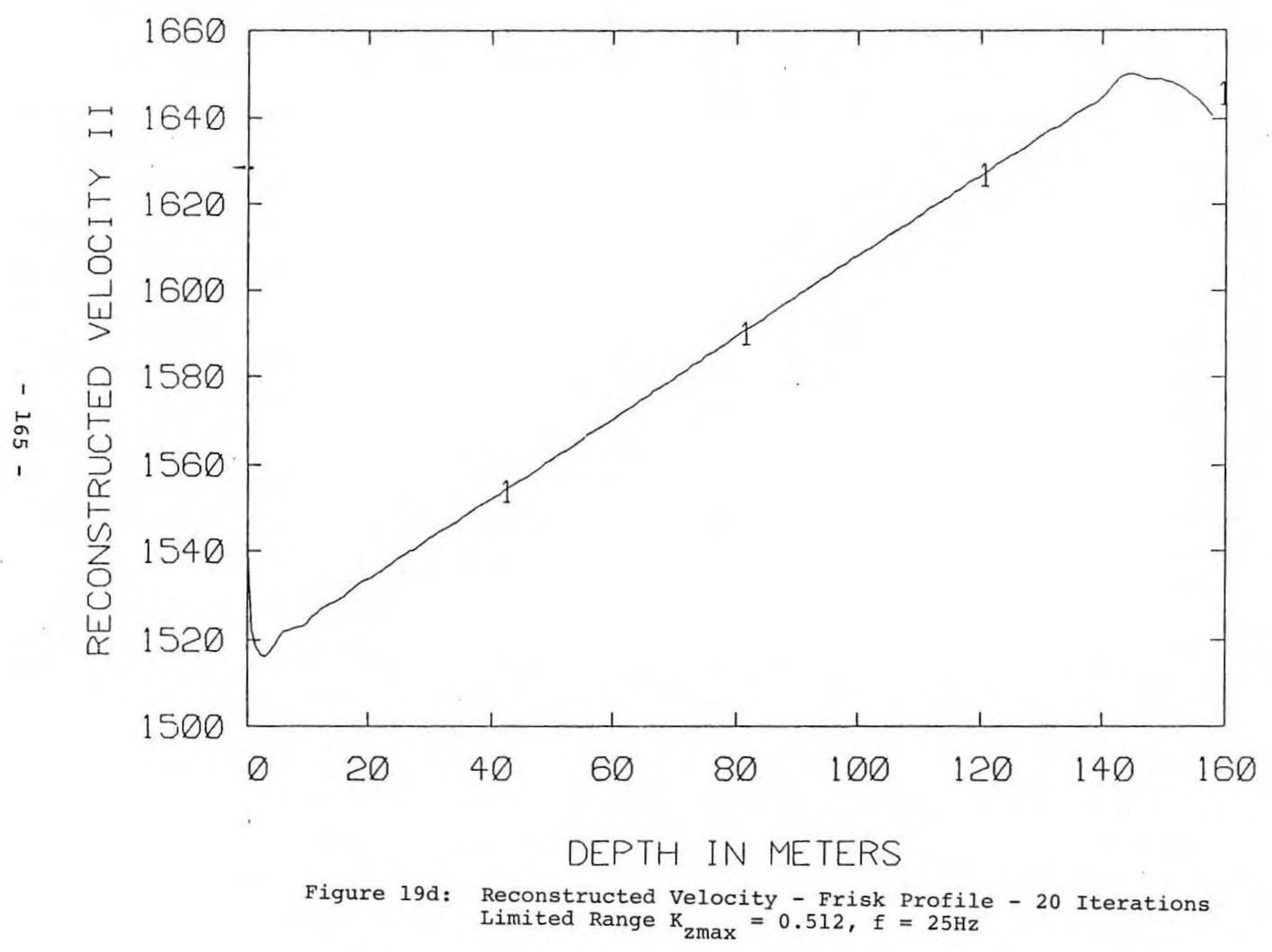




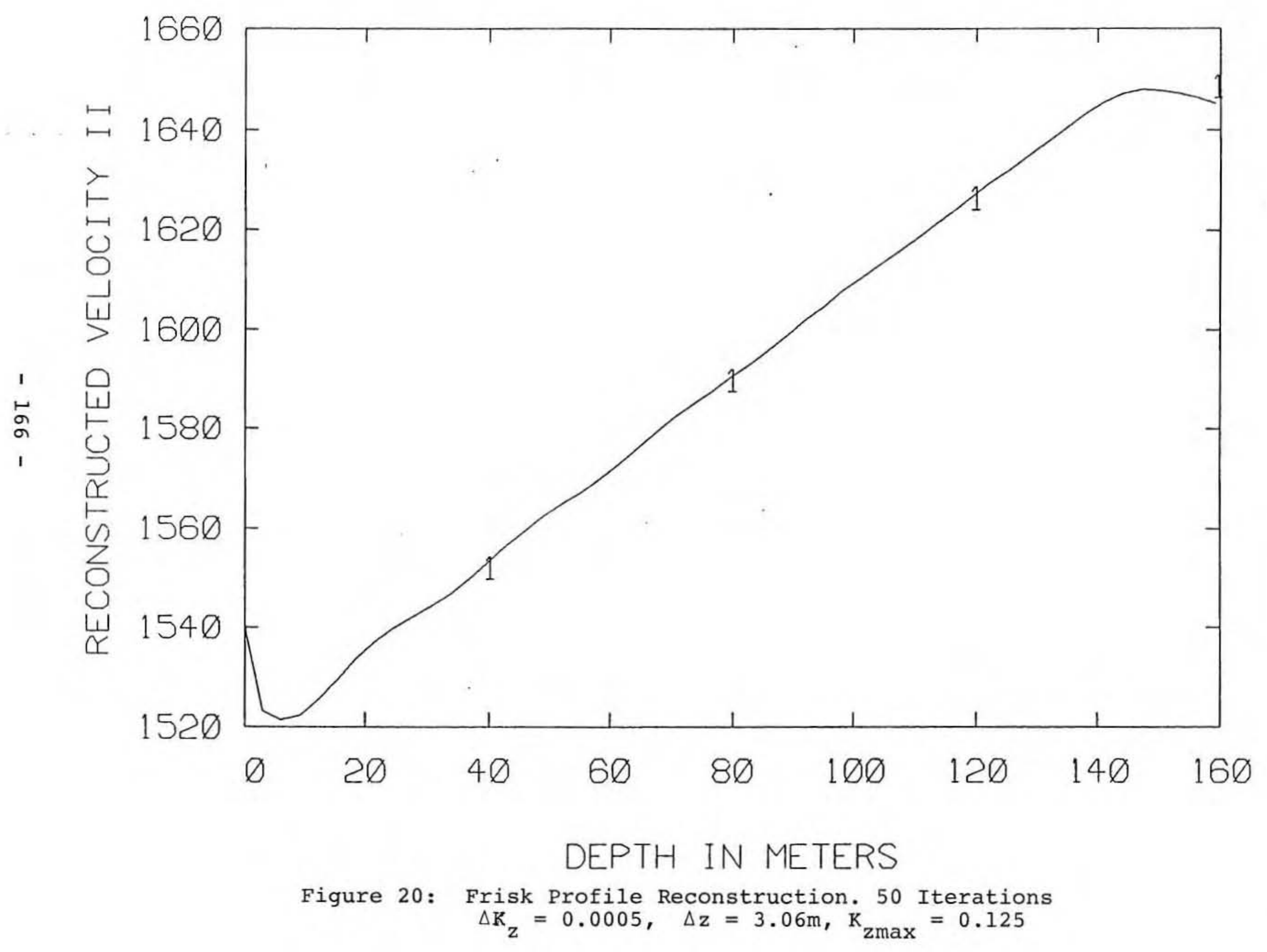




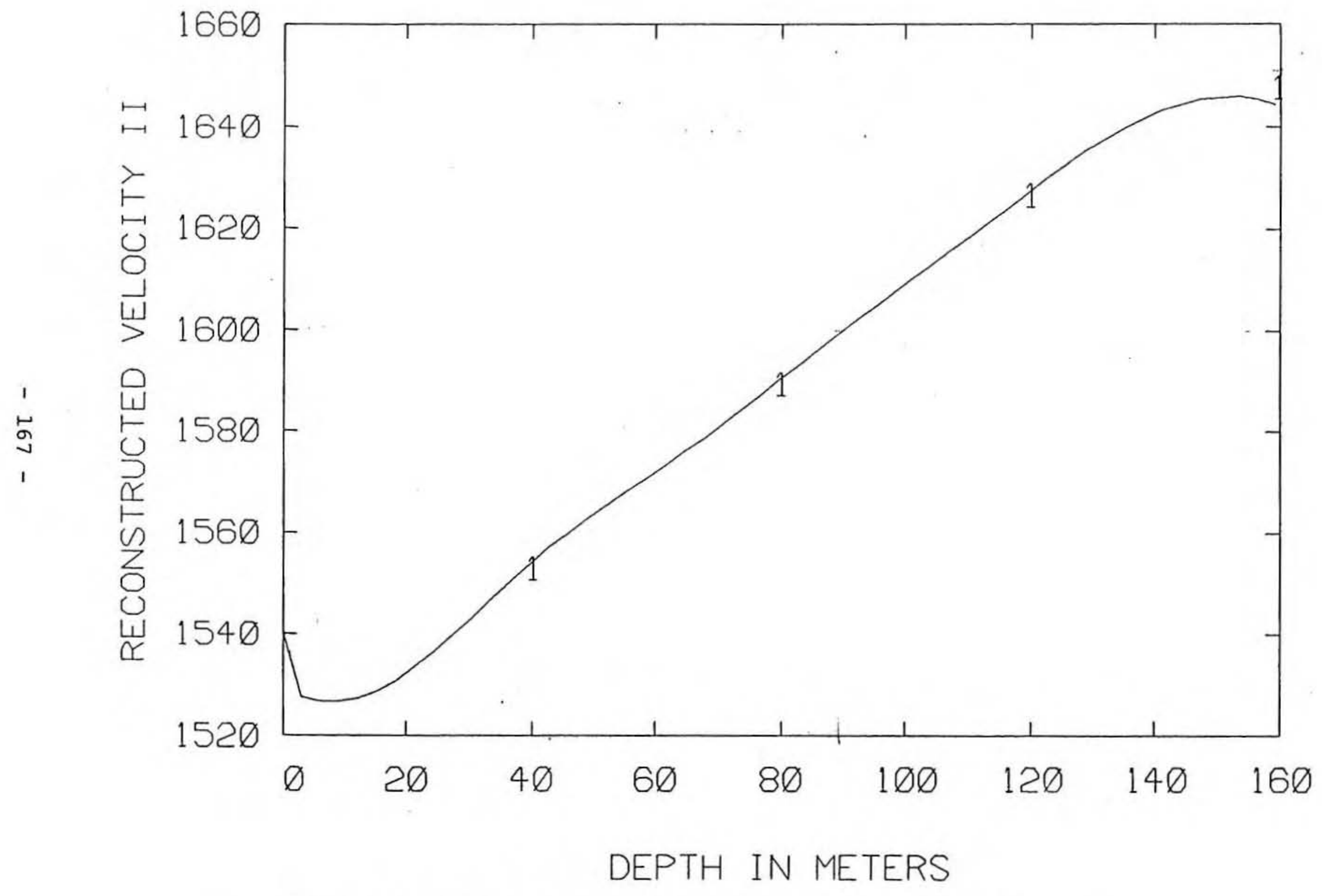

Figure 21: Frisk Profile Reconstruction. 40 Iterations Limited Range $\mathrm{K}_{z \max }=0.064-\Delta \mathrm{z}=3.06 \mathrm{~m}$ 


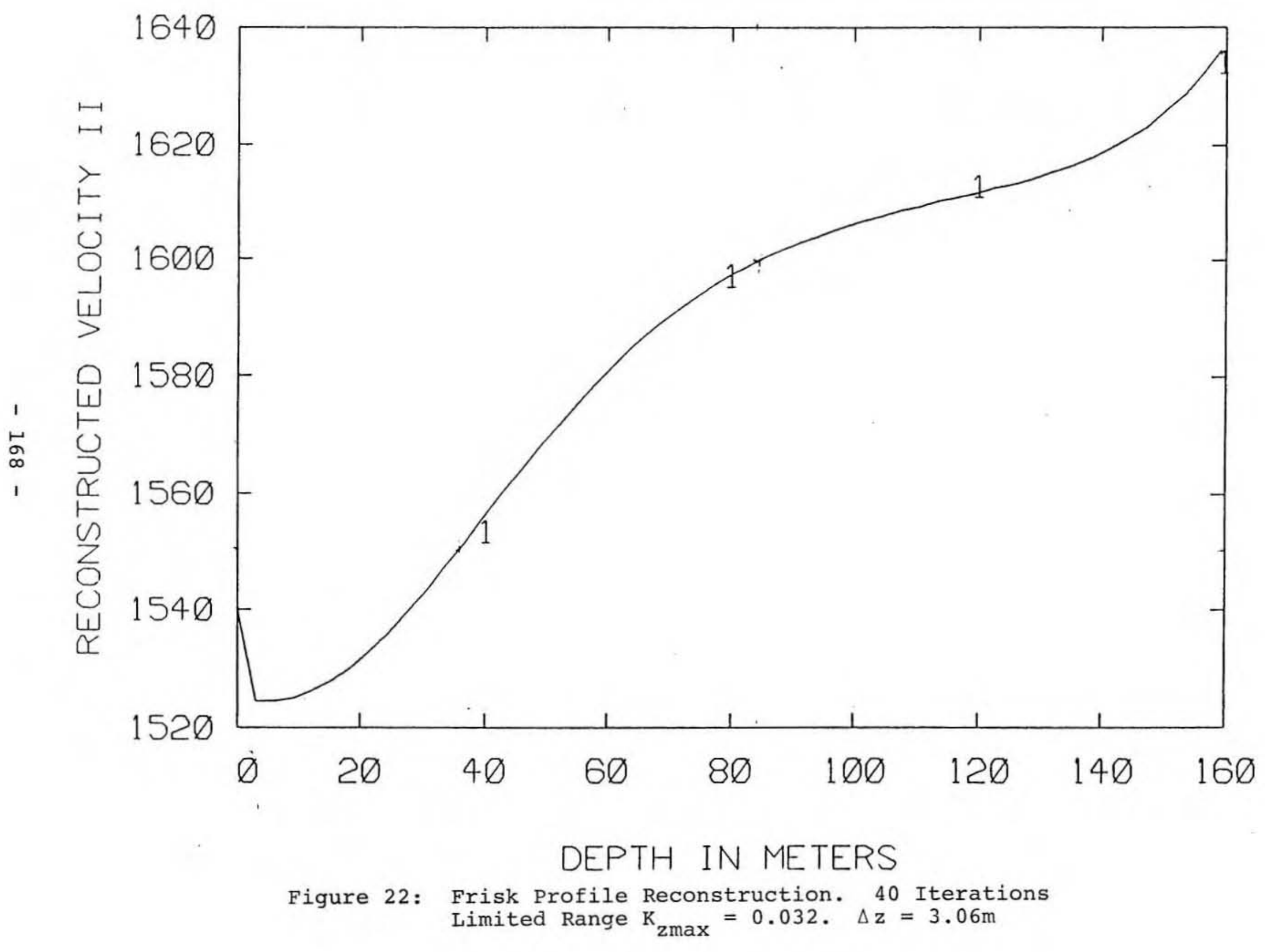




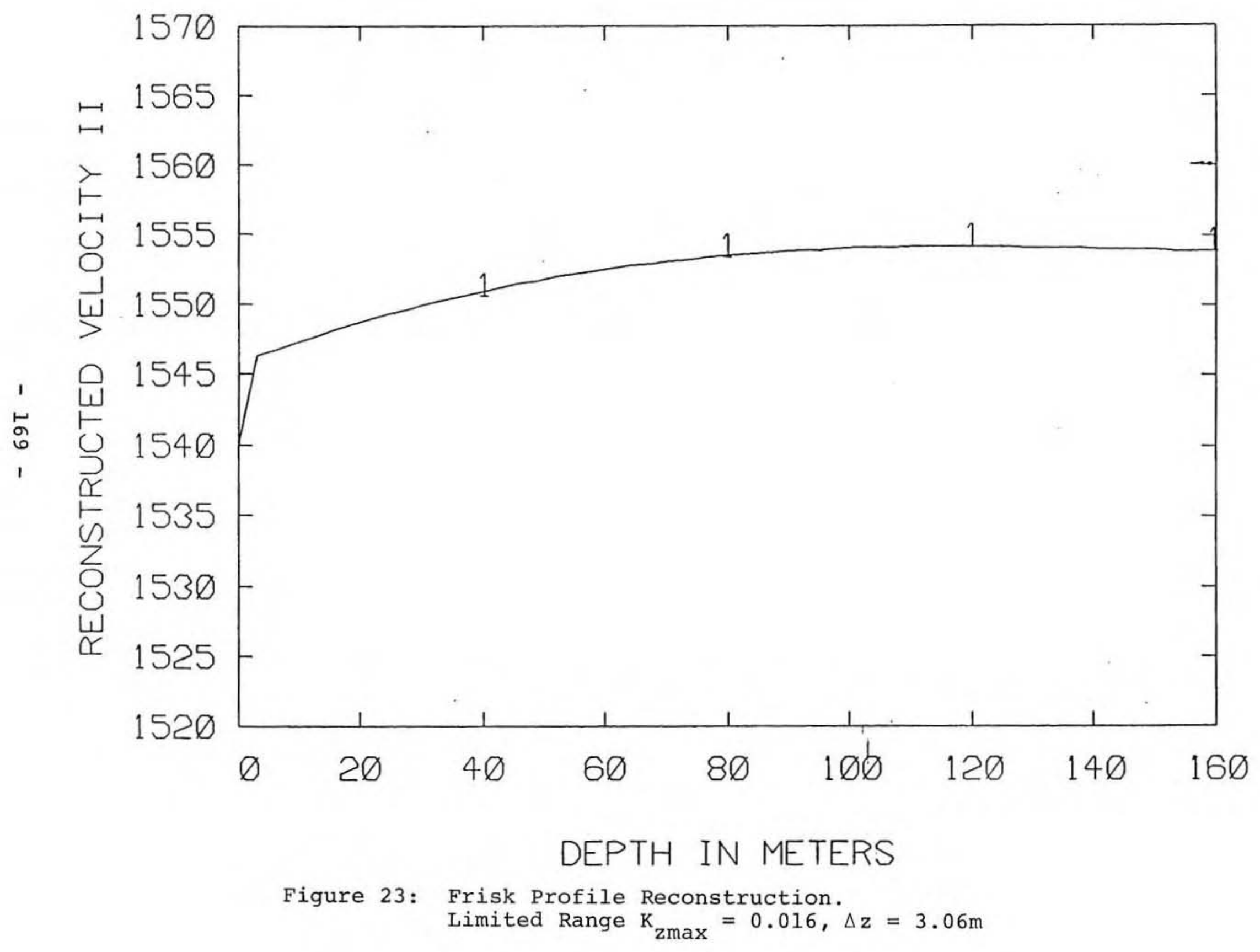




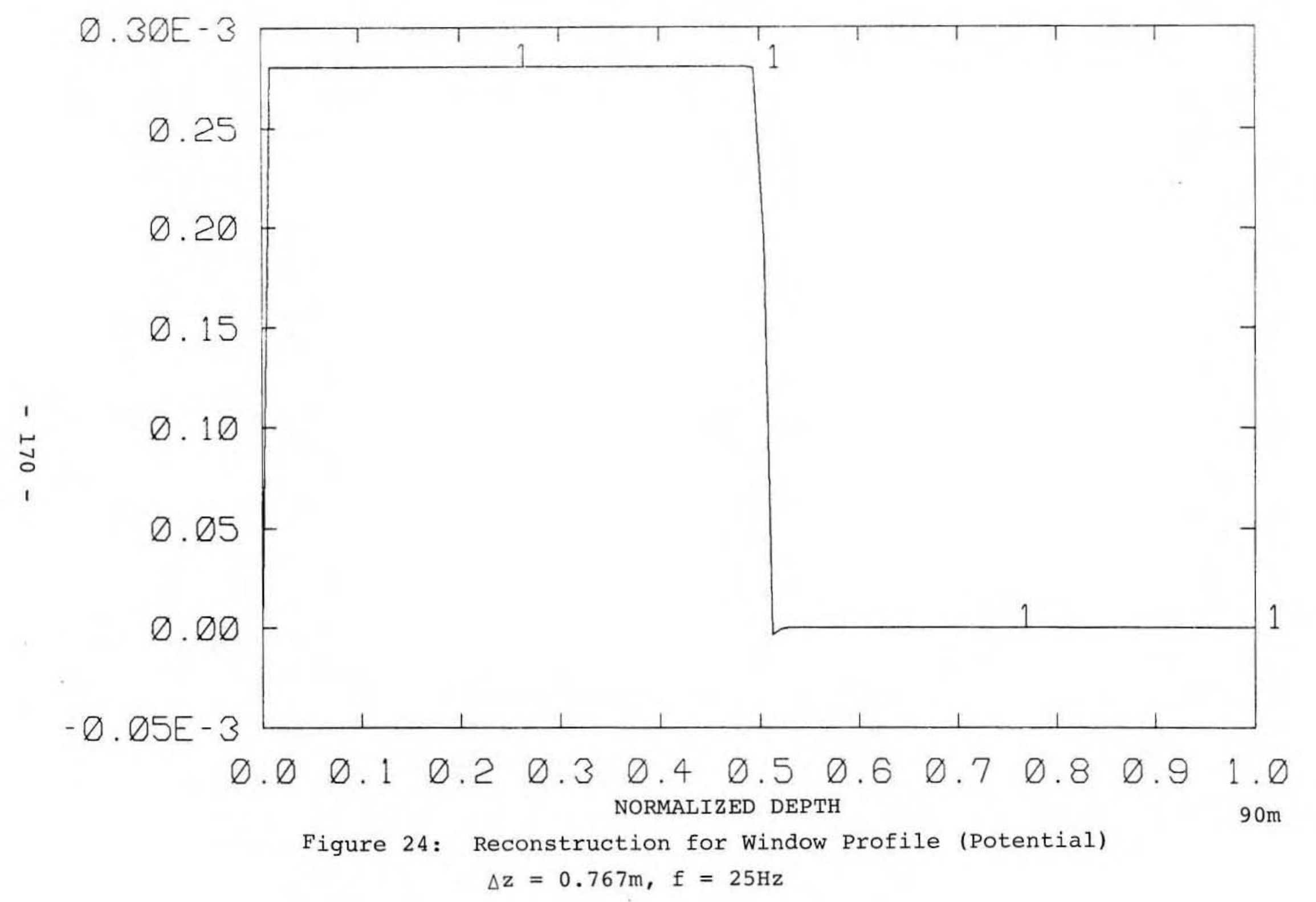




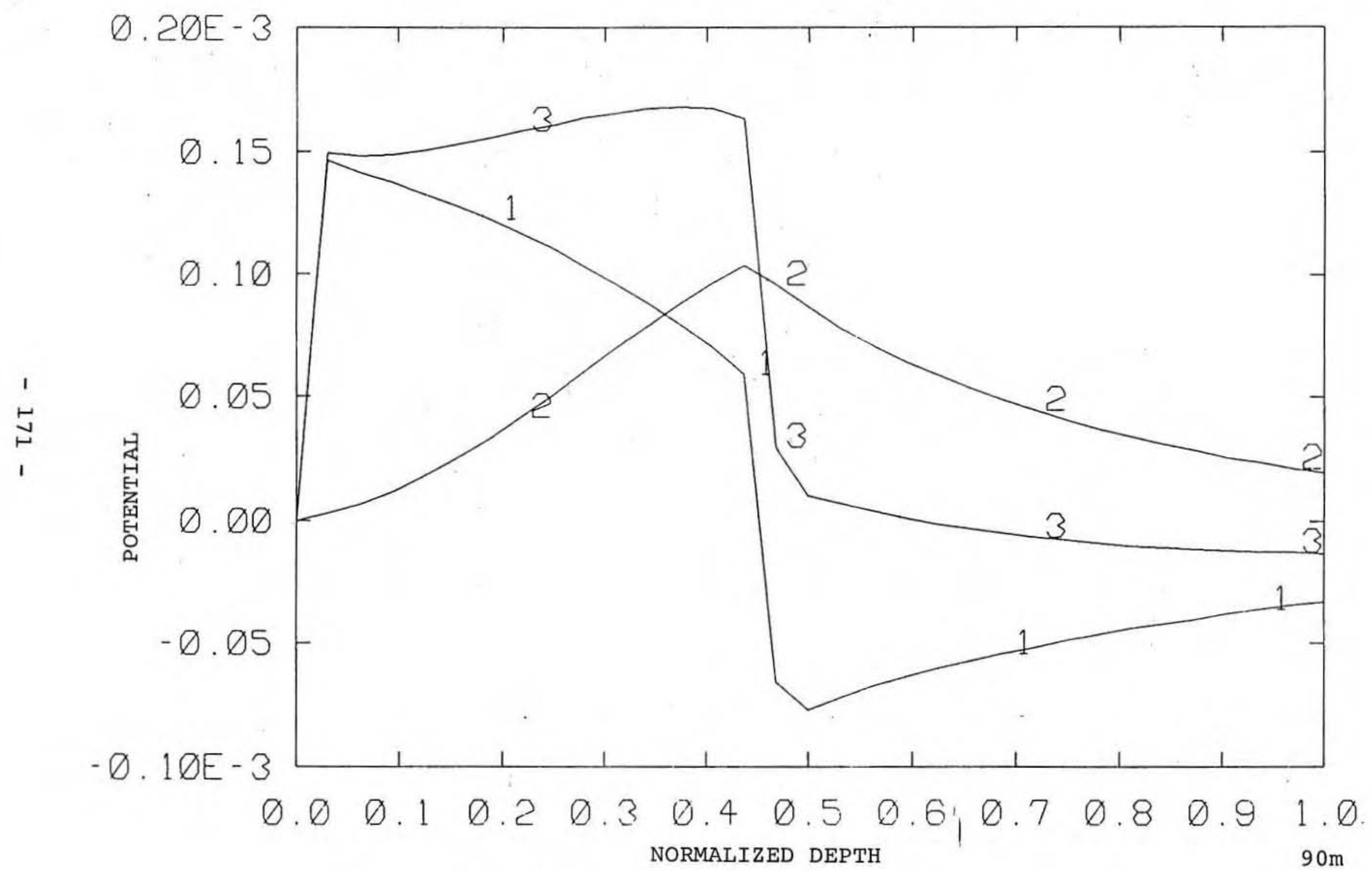

Figure 25a: Lossy Window Profile Reconstruction ( $\mathrm{W}=45 \mathrm{~m}) \quad \alpha=0.001 \mathrm{~dB} / \mathrm{m}$ 1: Born, 2: Correction, 3: Improved Born. $f=25 \mathrm{~Hz}$ 
1
$N$
$N$

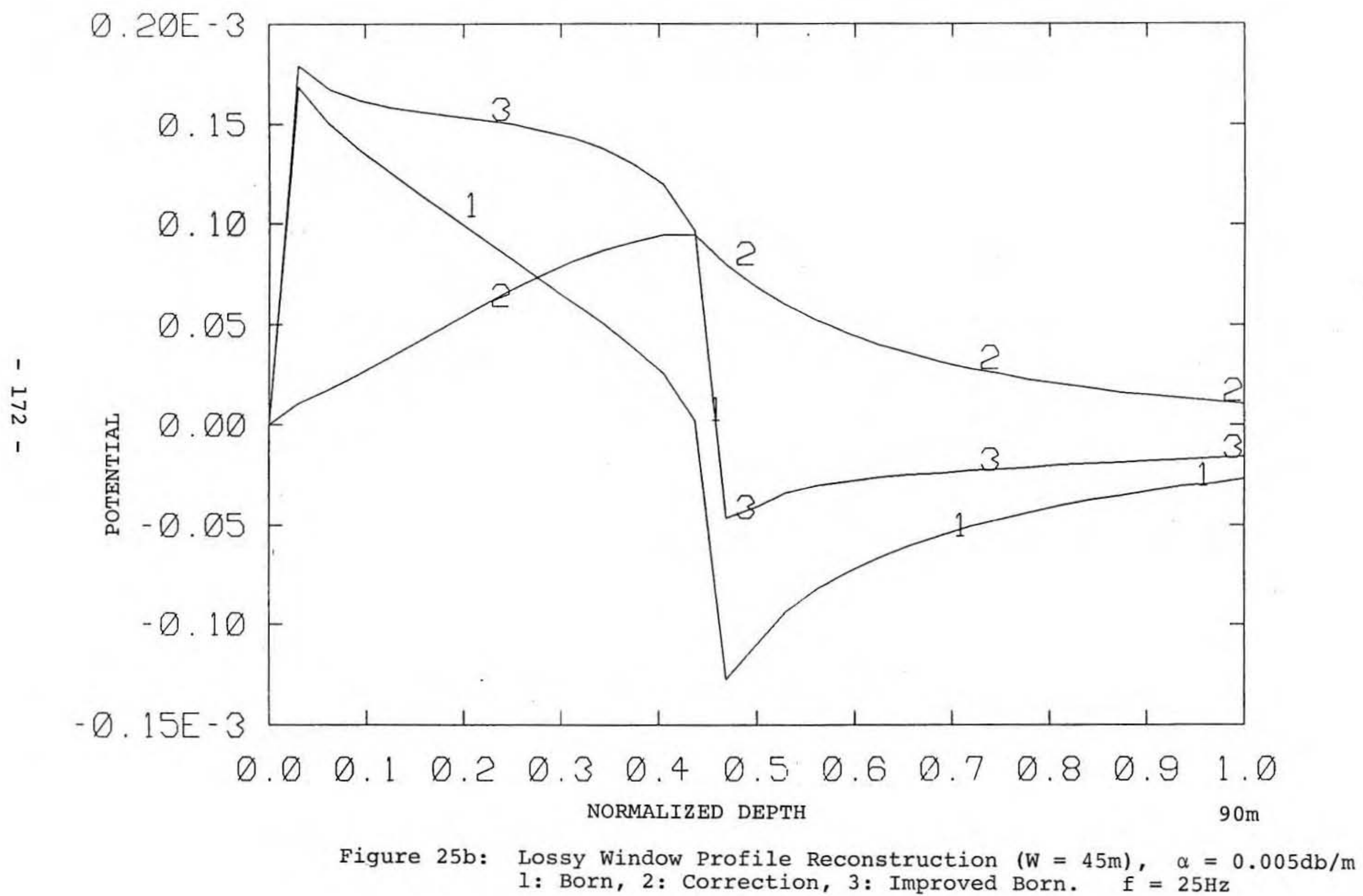




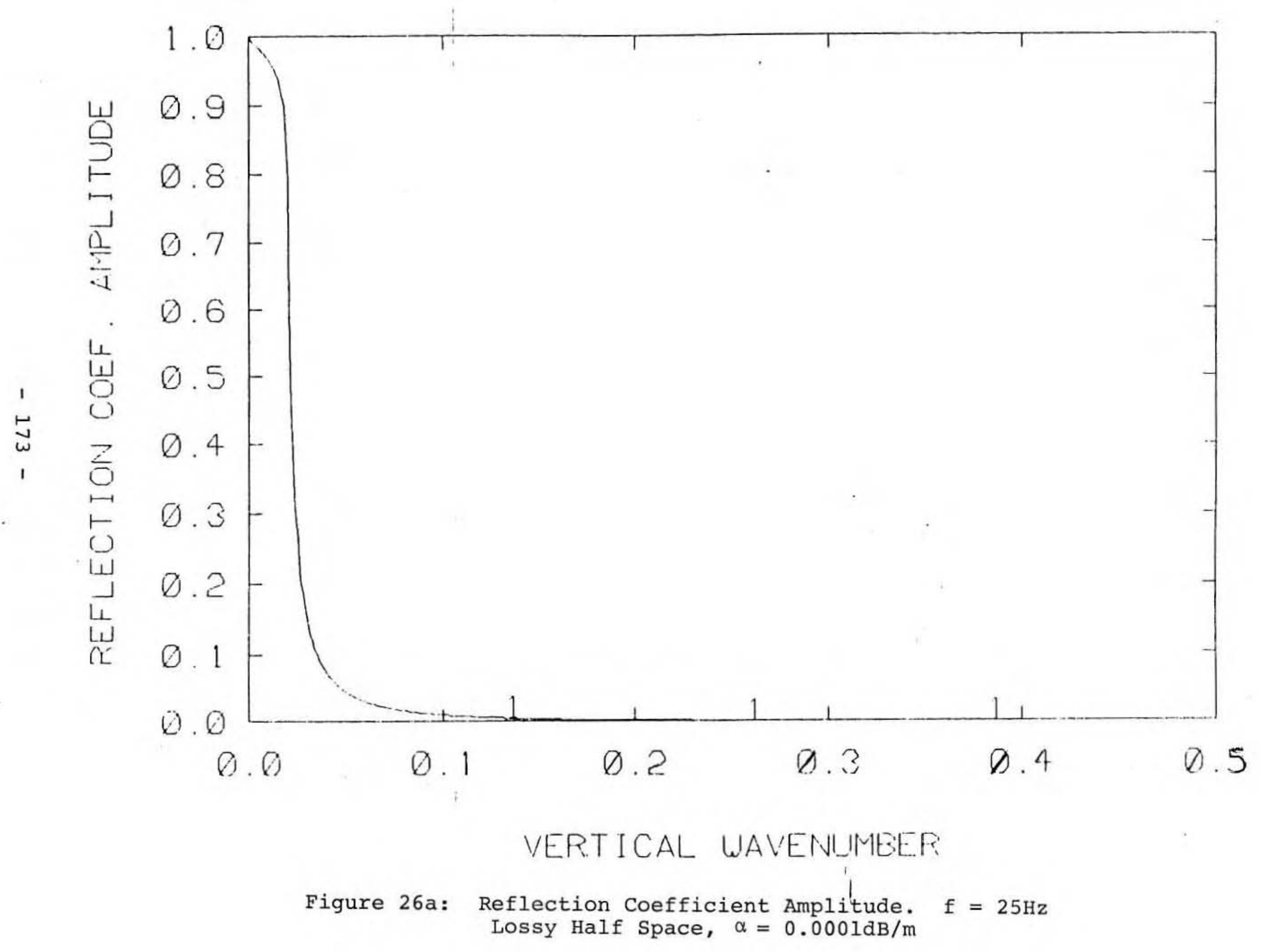




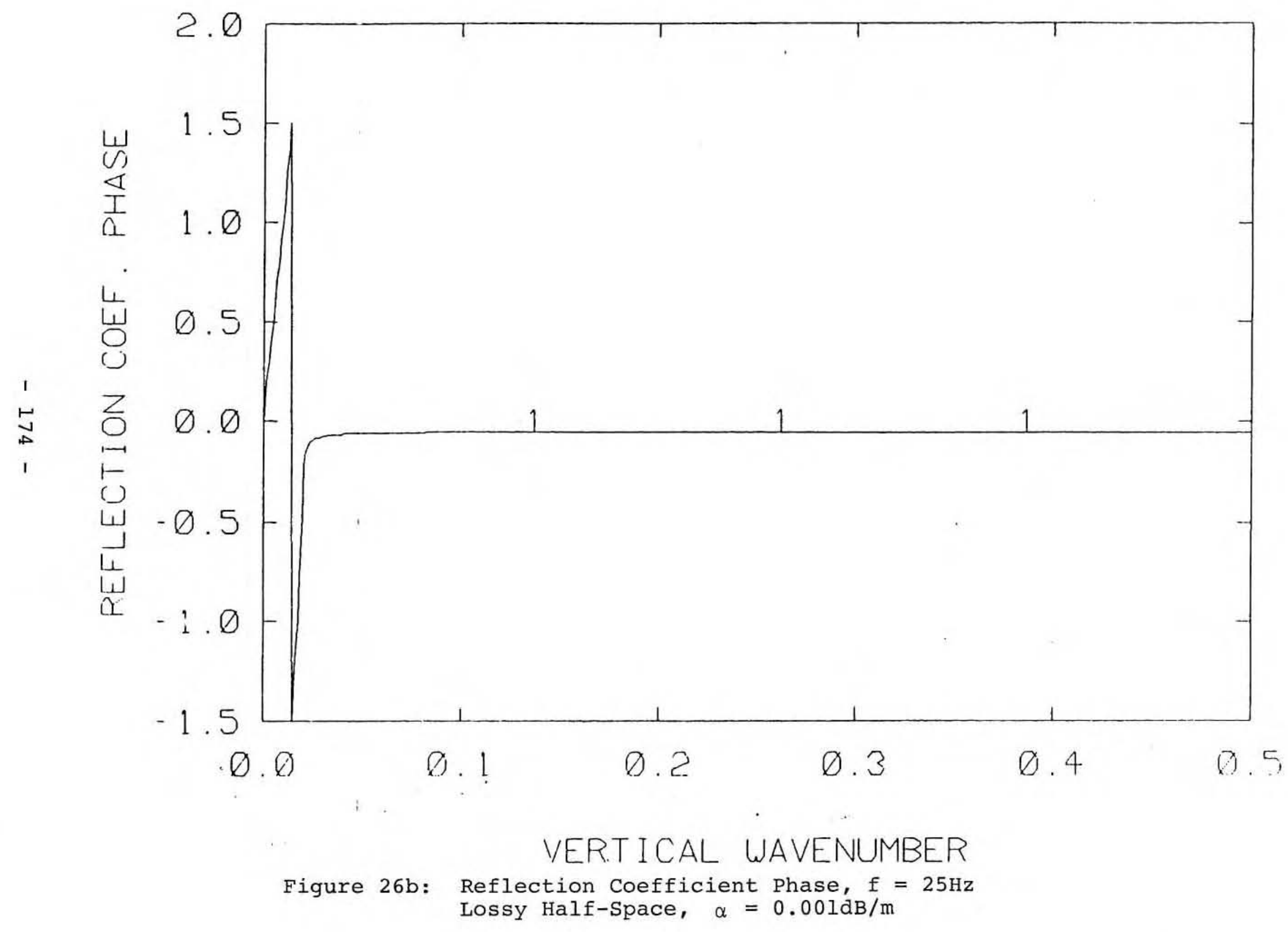




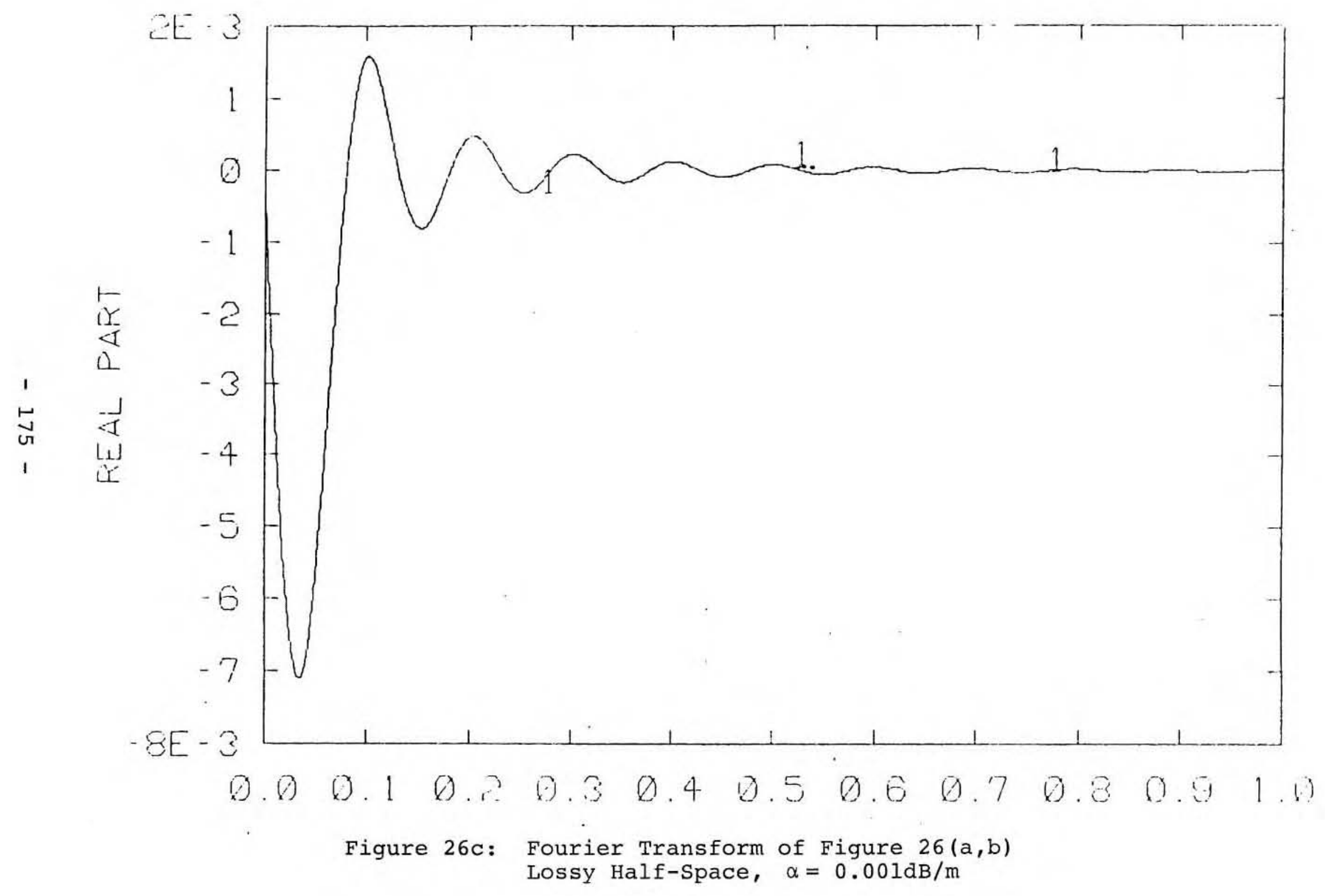




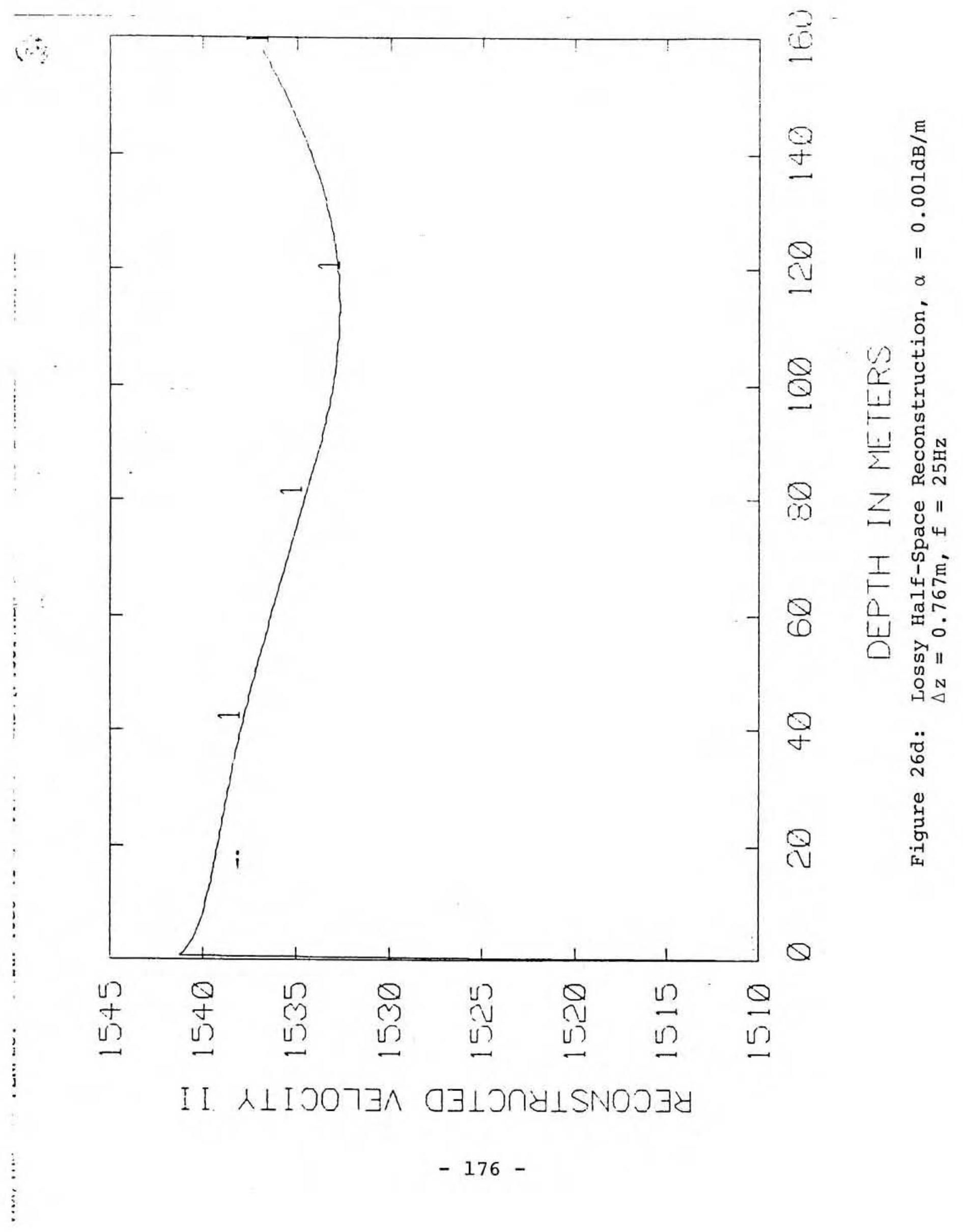




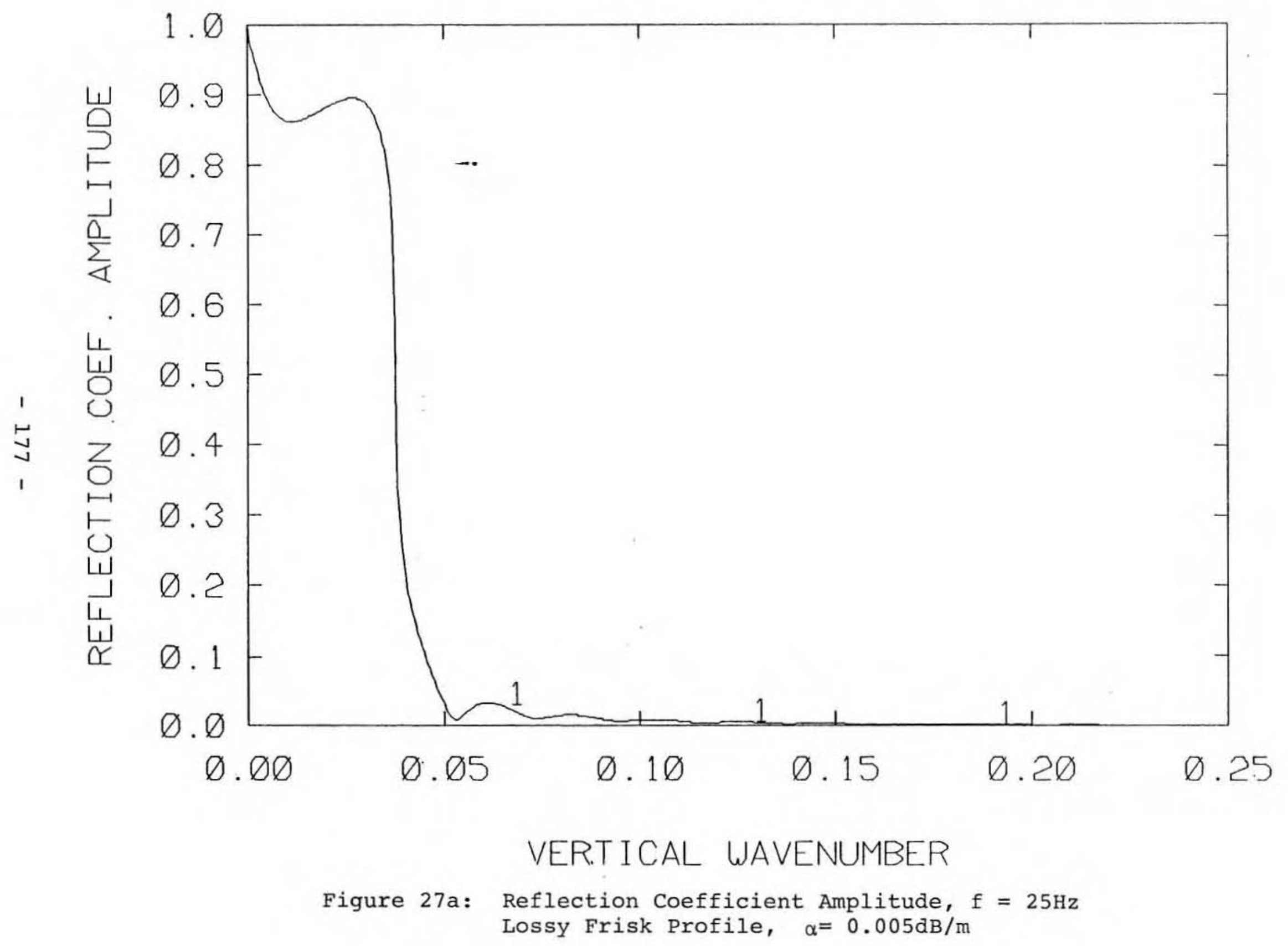




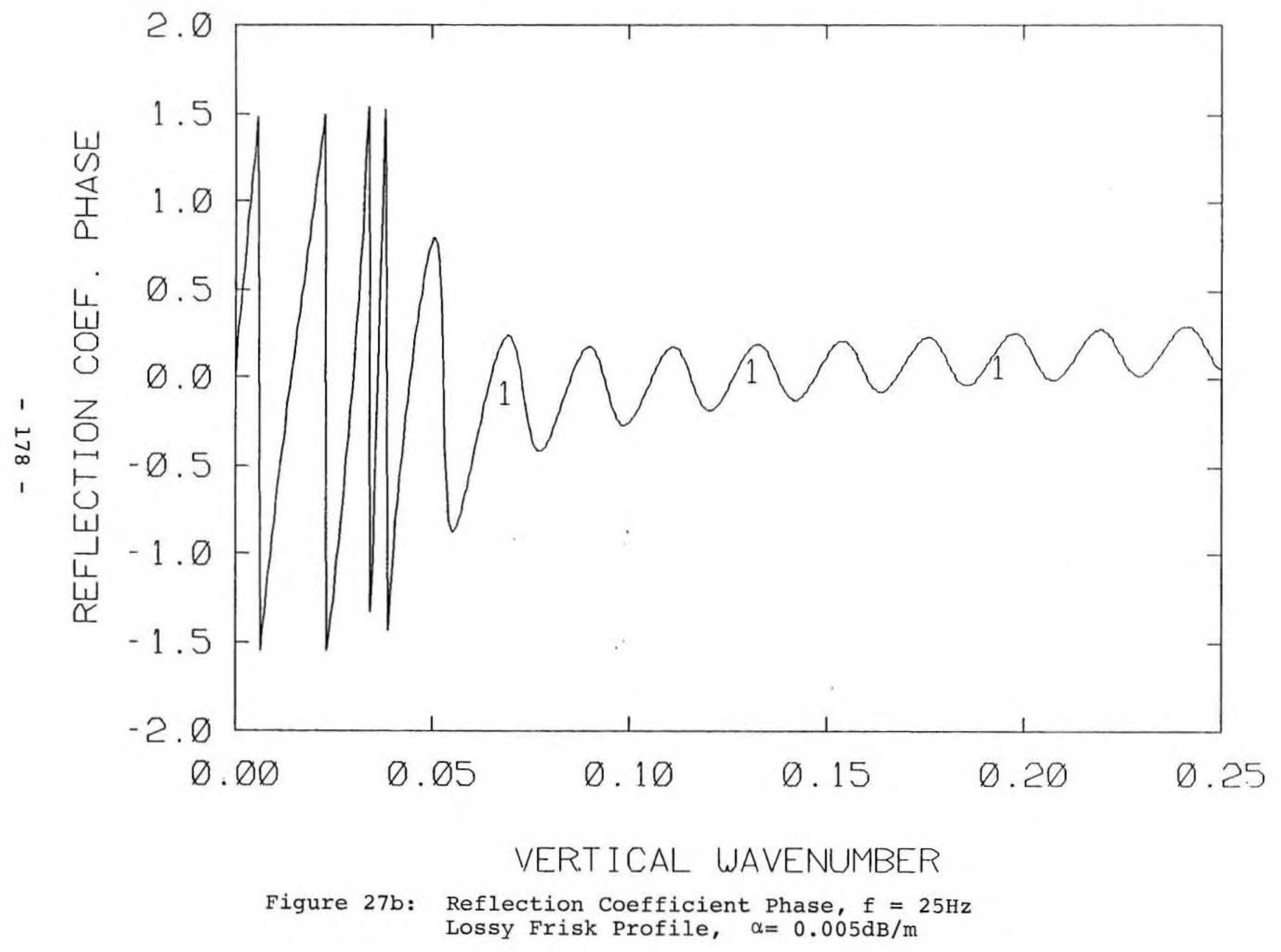




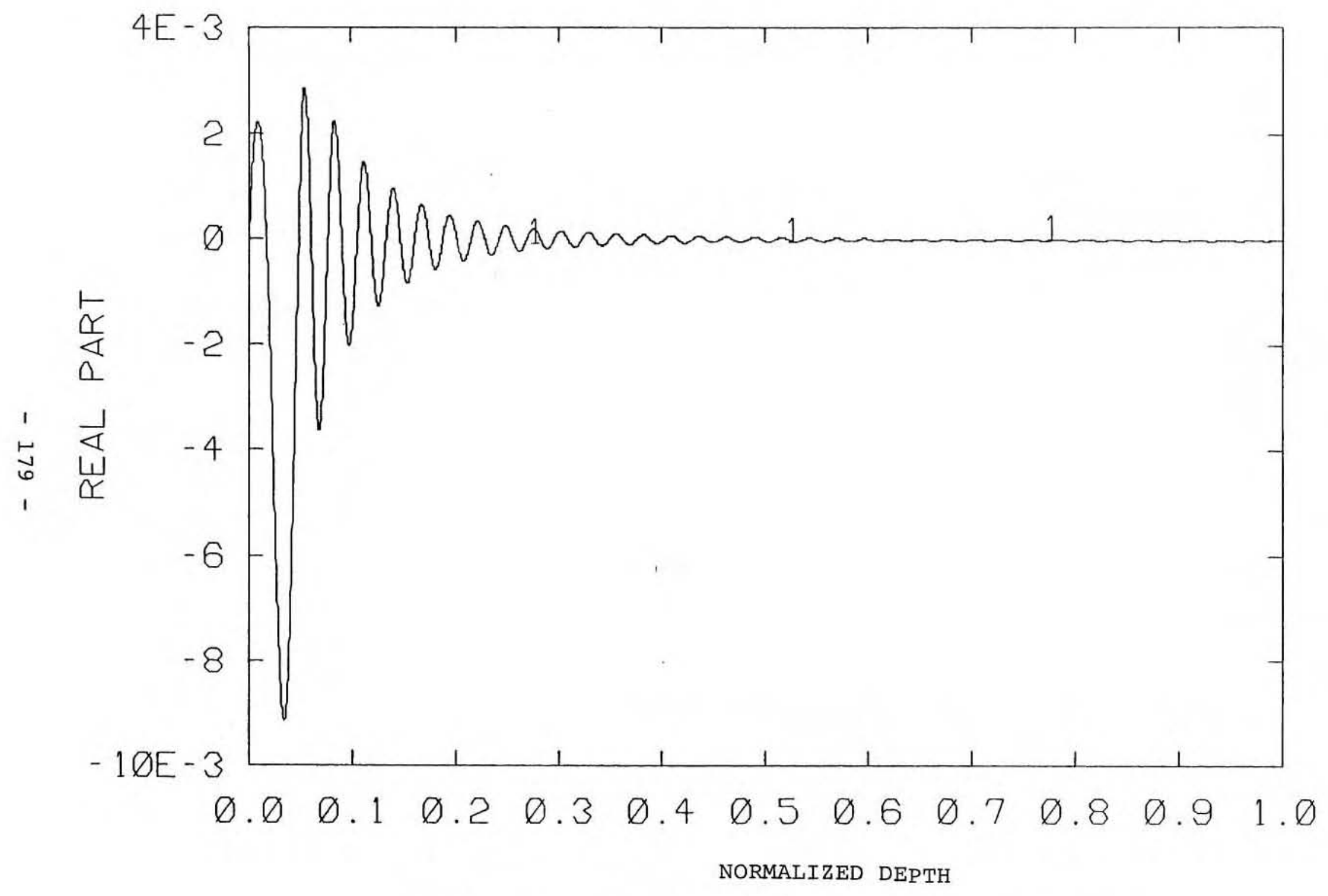

Figure 27c: Fourier Transform of Figure $27(a, b)$ 


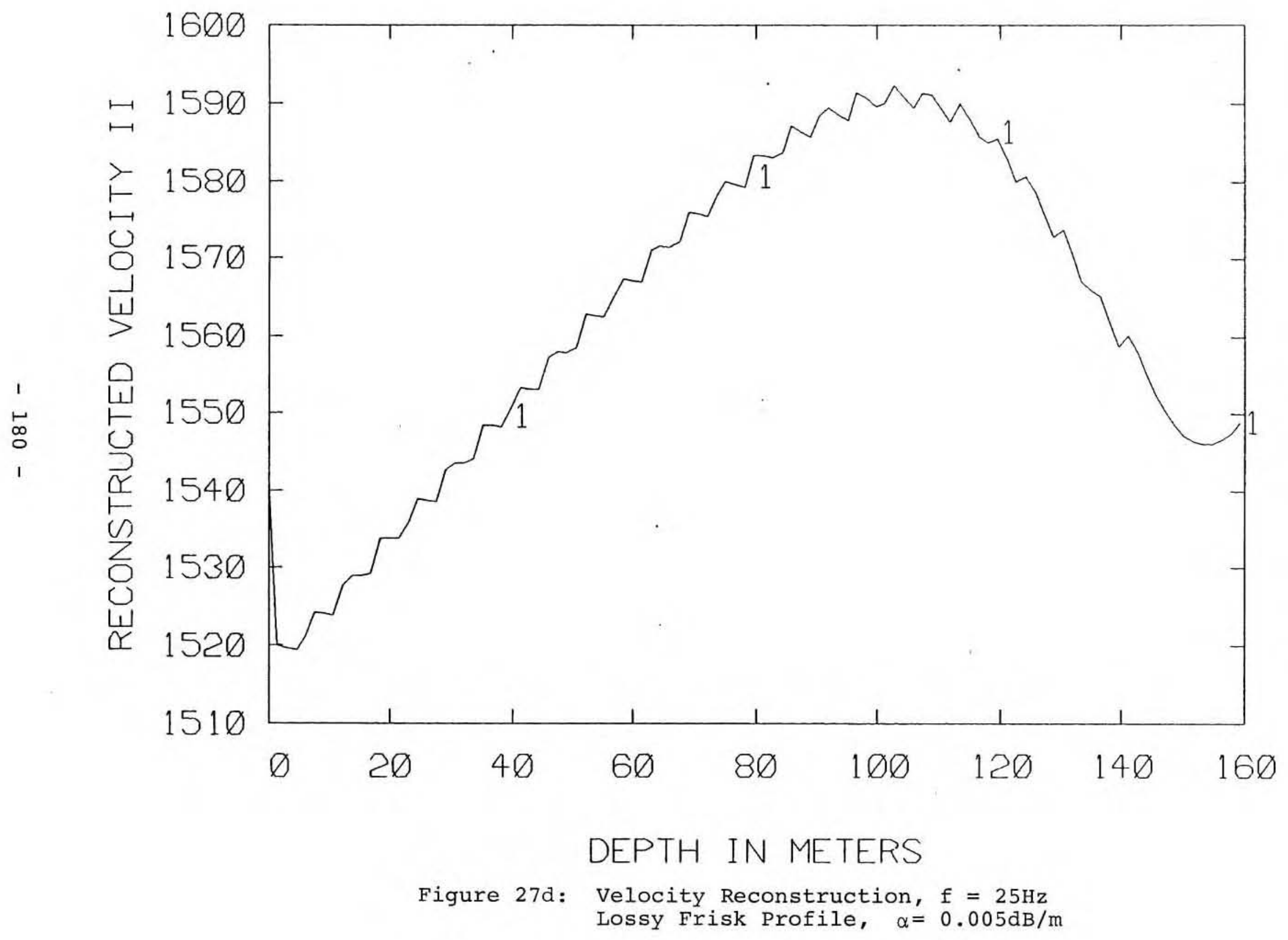




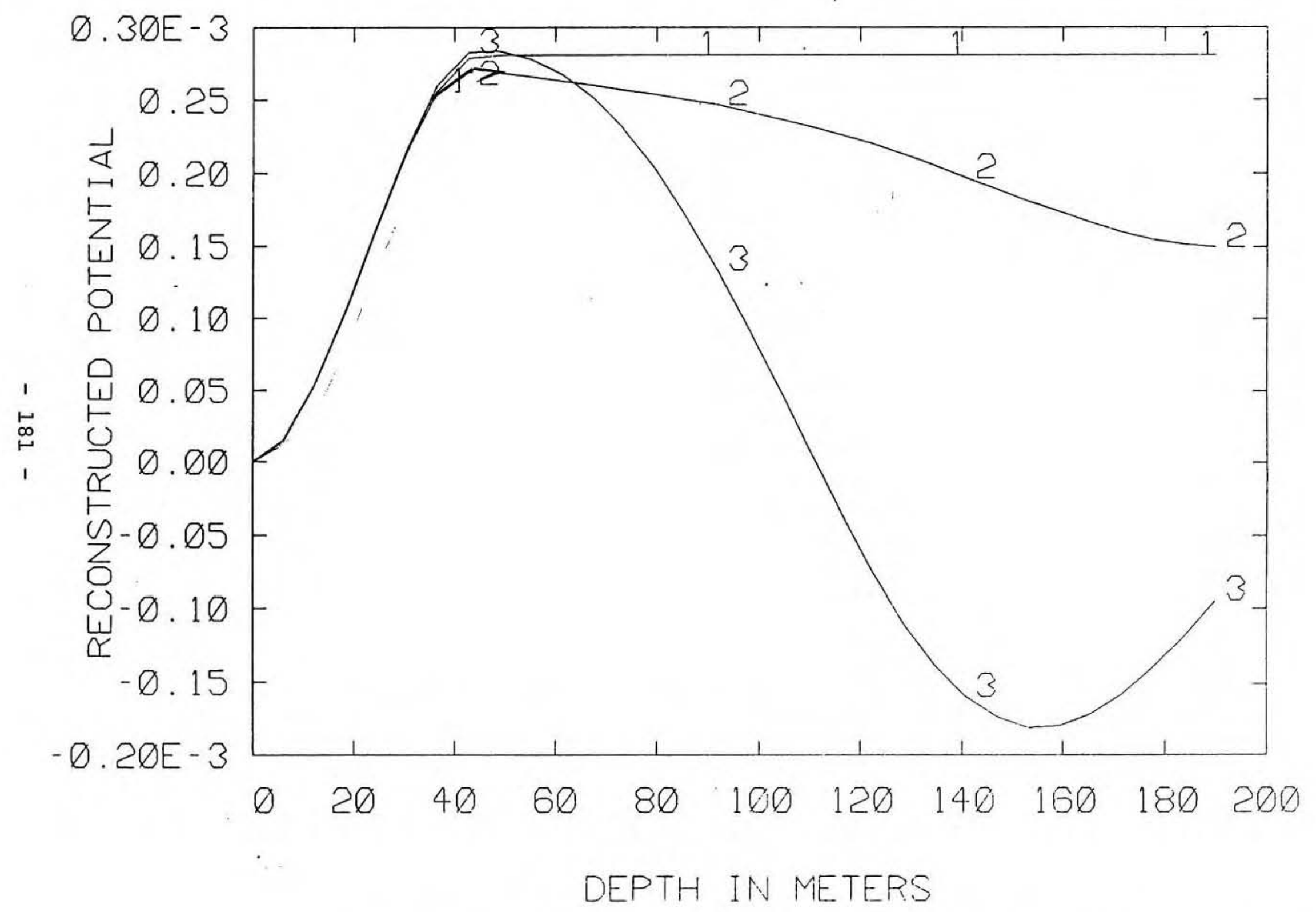

Figure 28: Stickler Profile Reconstruction. $\Delta z=6.03 \mathrm{~m}, \mathrm{~L}=40 \mathrm{~m}, \mathrm{f}=25 \mathrm{~Hz}$ 1: Original Profile, 2: Finite Difference, 3: Improved Born 


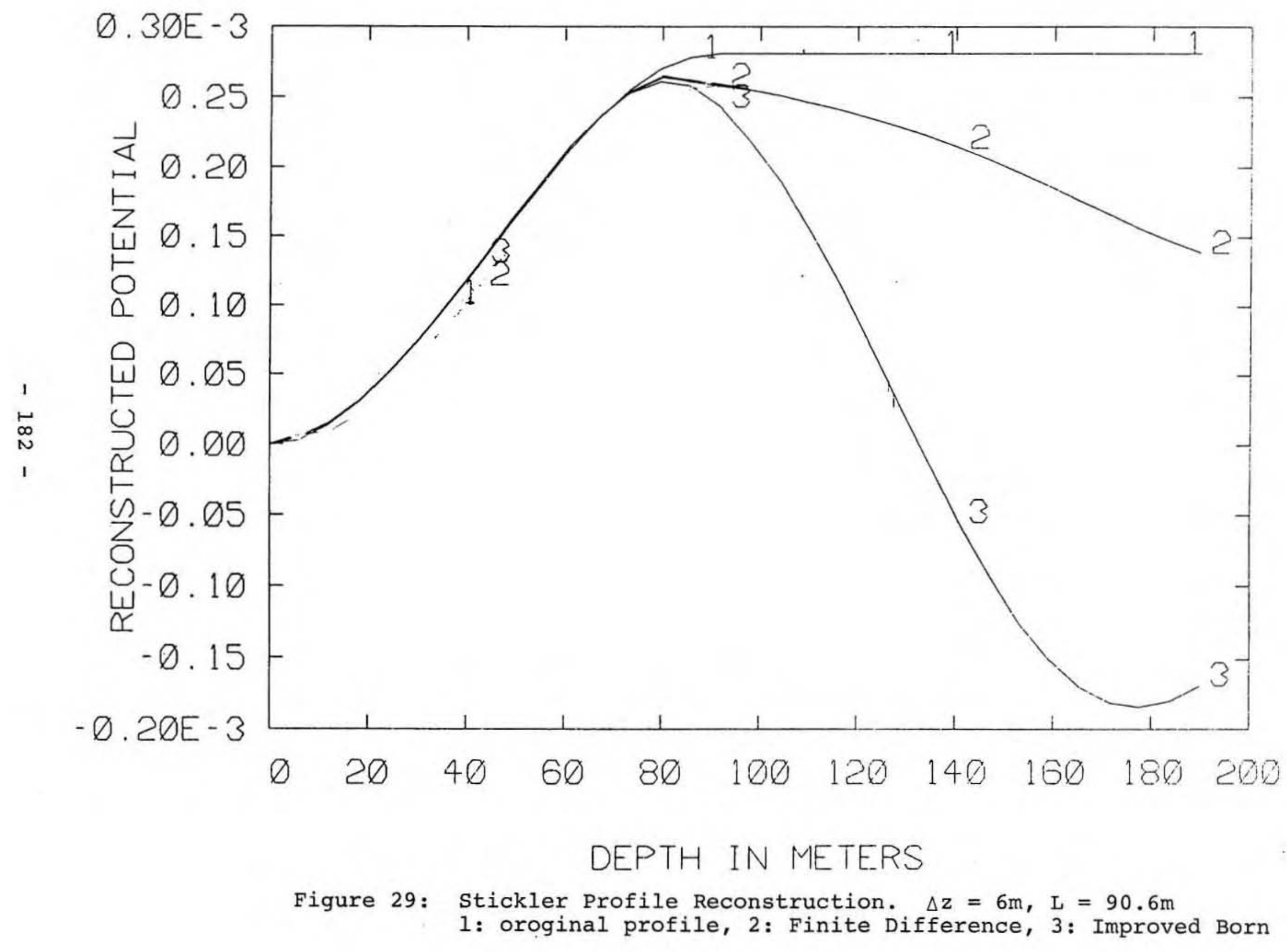




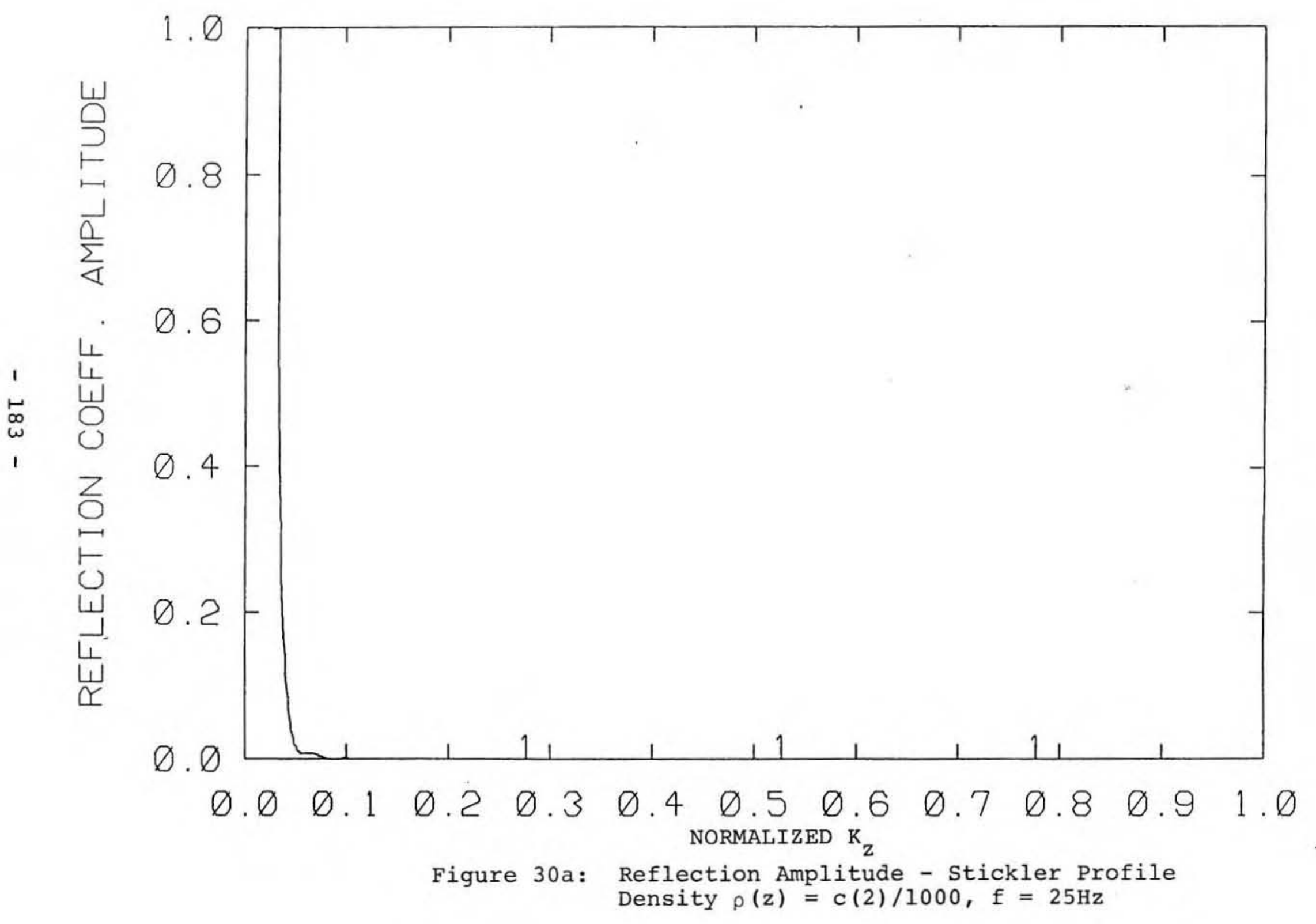




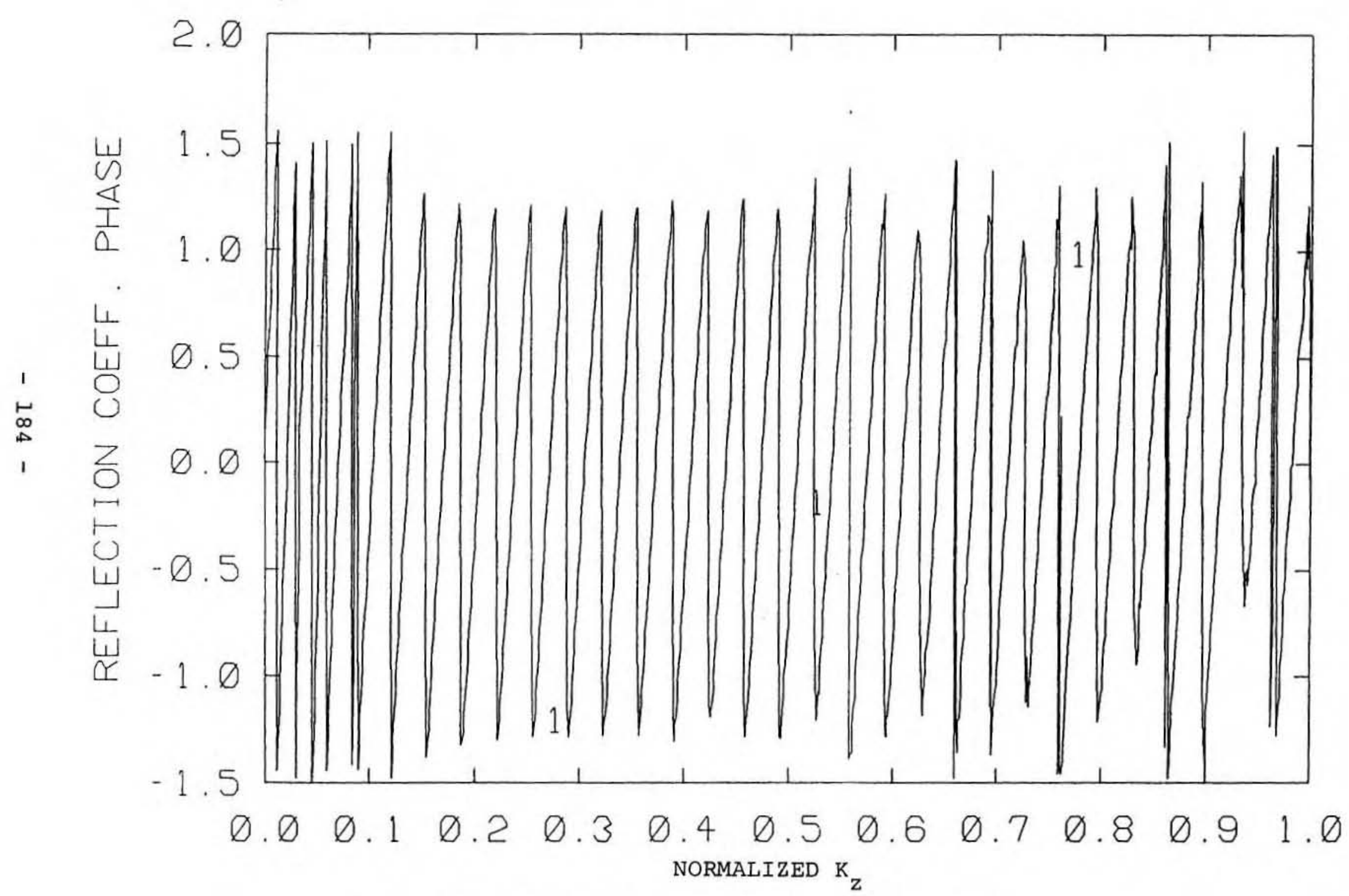

Figure 30b: Reflection Phase Stickler Profile

Density $\rho(z)=c(z) / 1000, f=25 \mathrm{~Hz}$ 


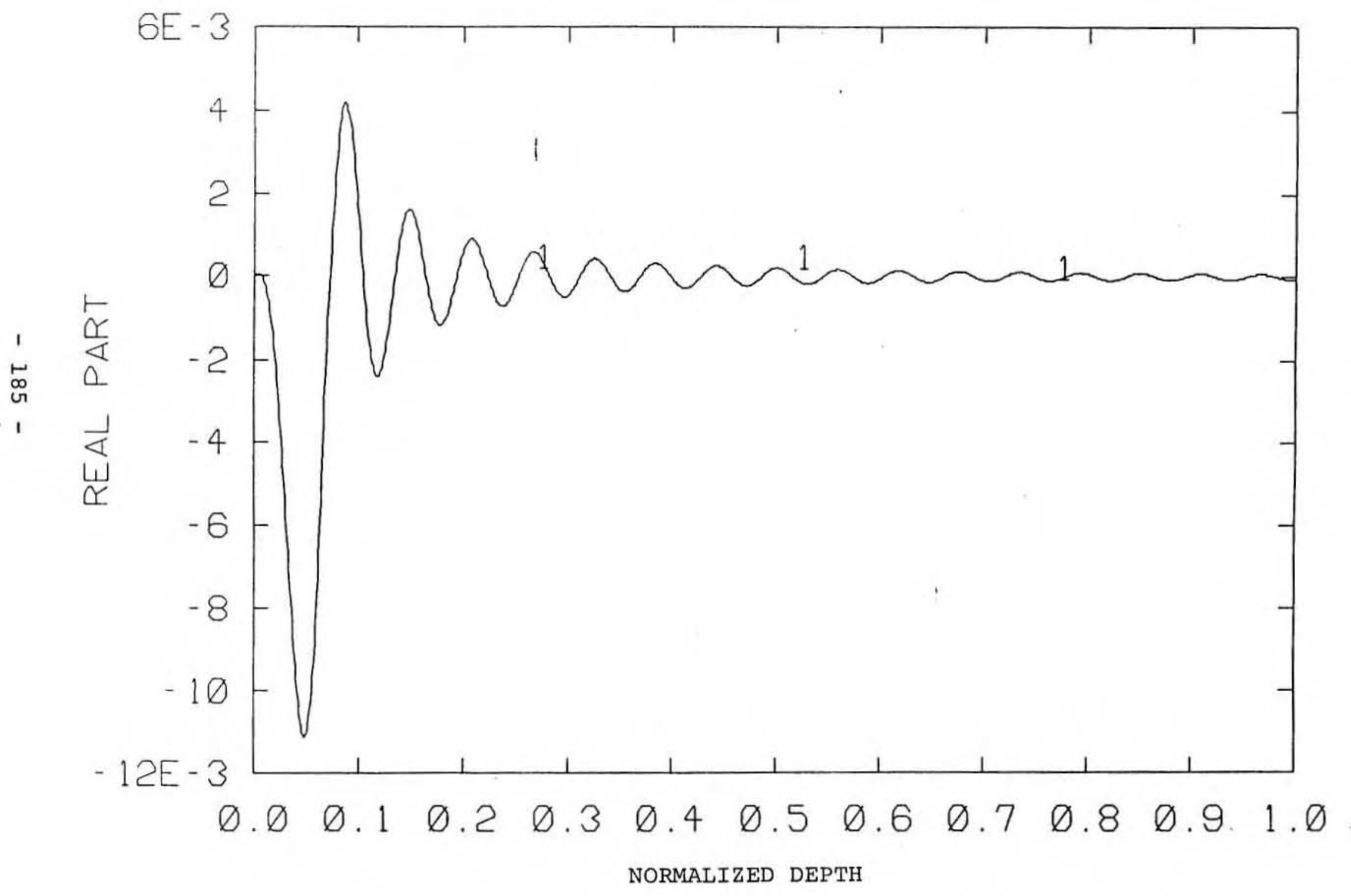

Figure 30c: Fourier Transform of Figure $30(a, b)$ 


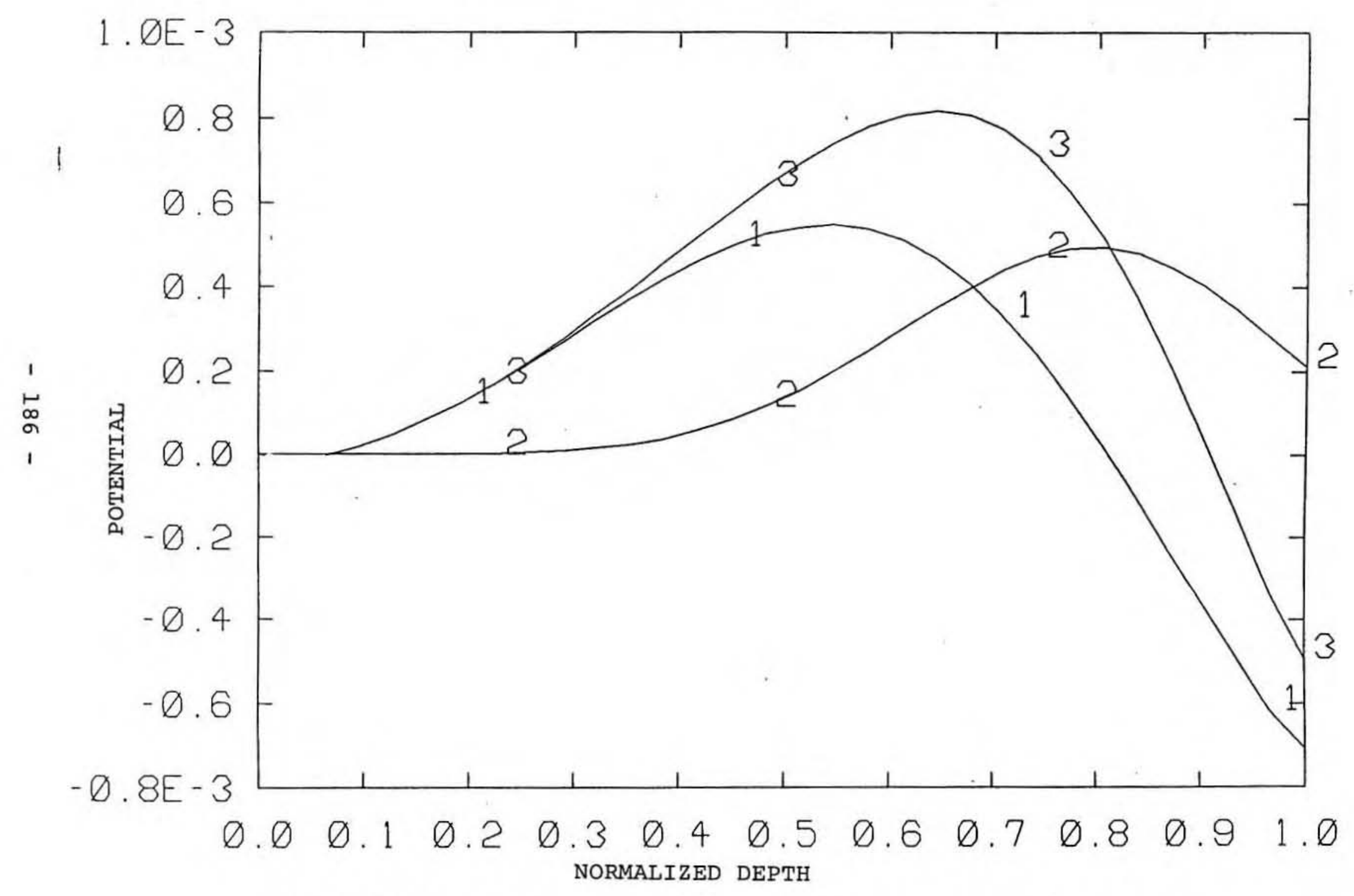

Figure 30d: Density-Dependent Potential Reconstruction $v_{i}(z)$ Born: 1, Correction: 2, Improved Born: 3

$f=25 \mathrm{~Hz}, \quad \rho(z)=c(z) / 1000$ 


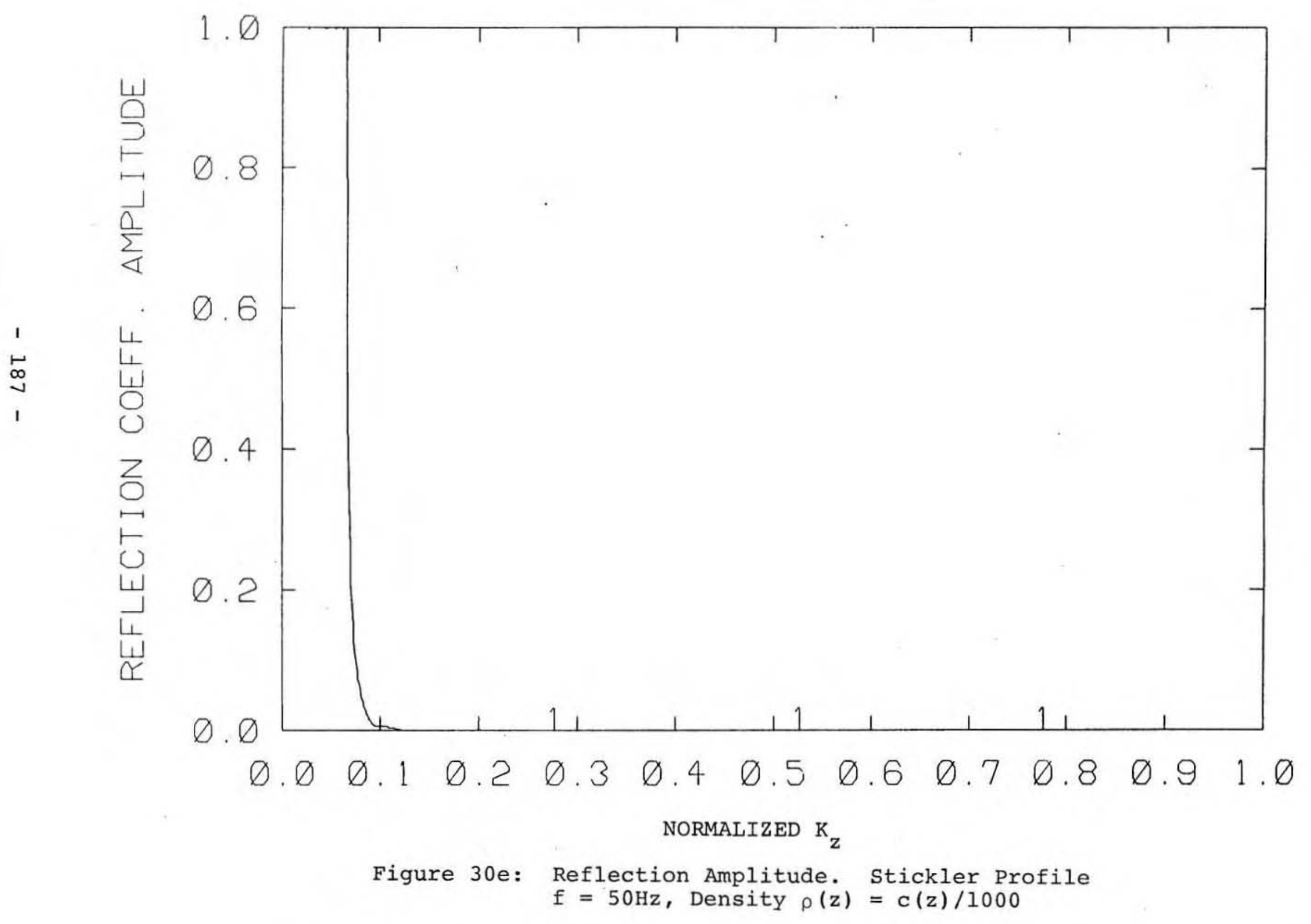




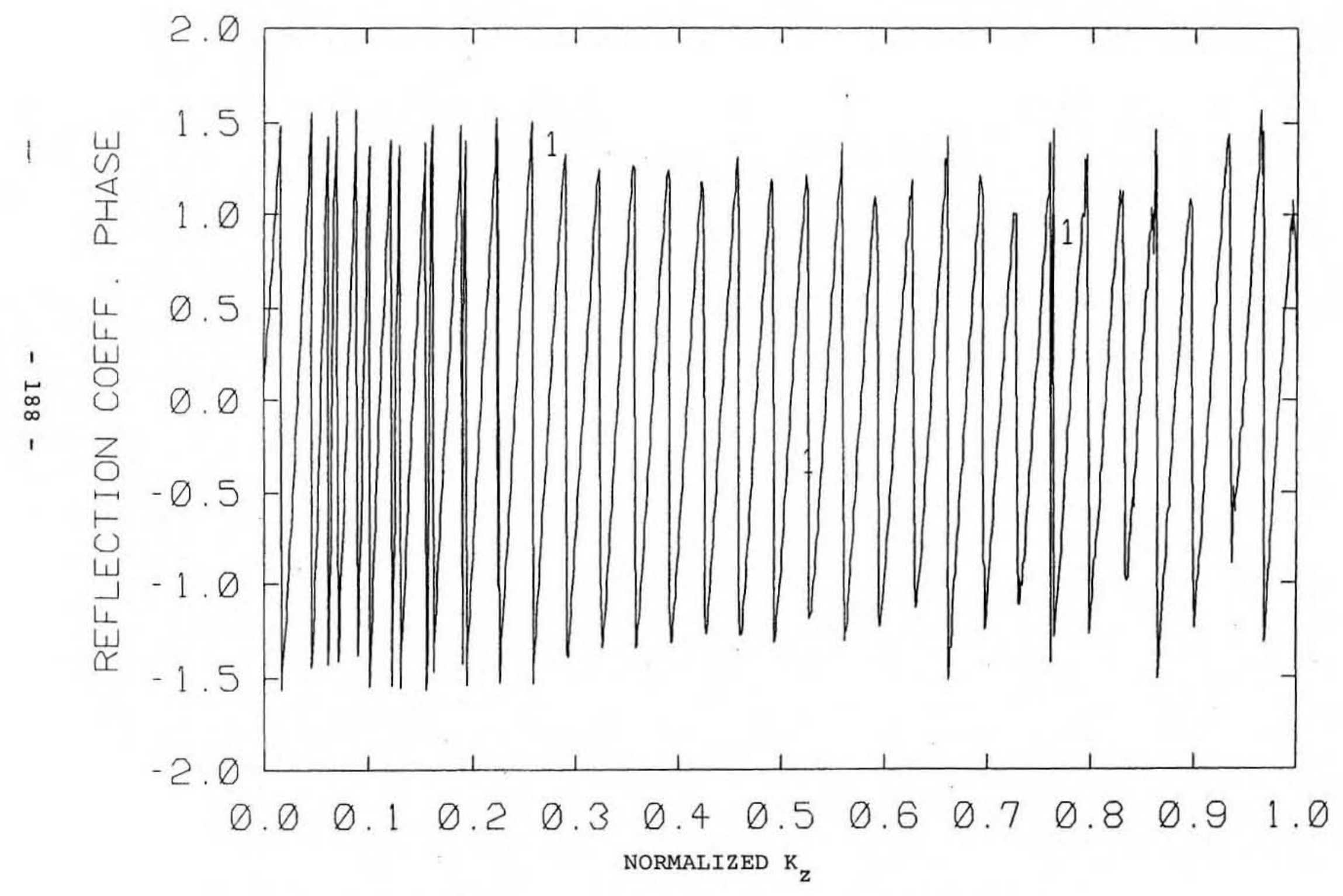

Figure 30f: Reflection Phase. Stickler Profile

$f=50 \mathrm{~Hz}, \quad \rho(z)=c(z) / 1000$ 


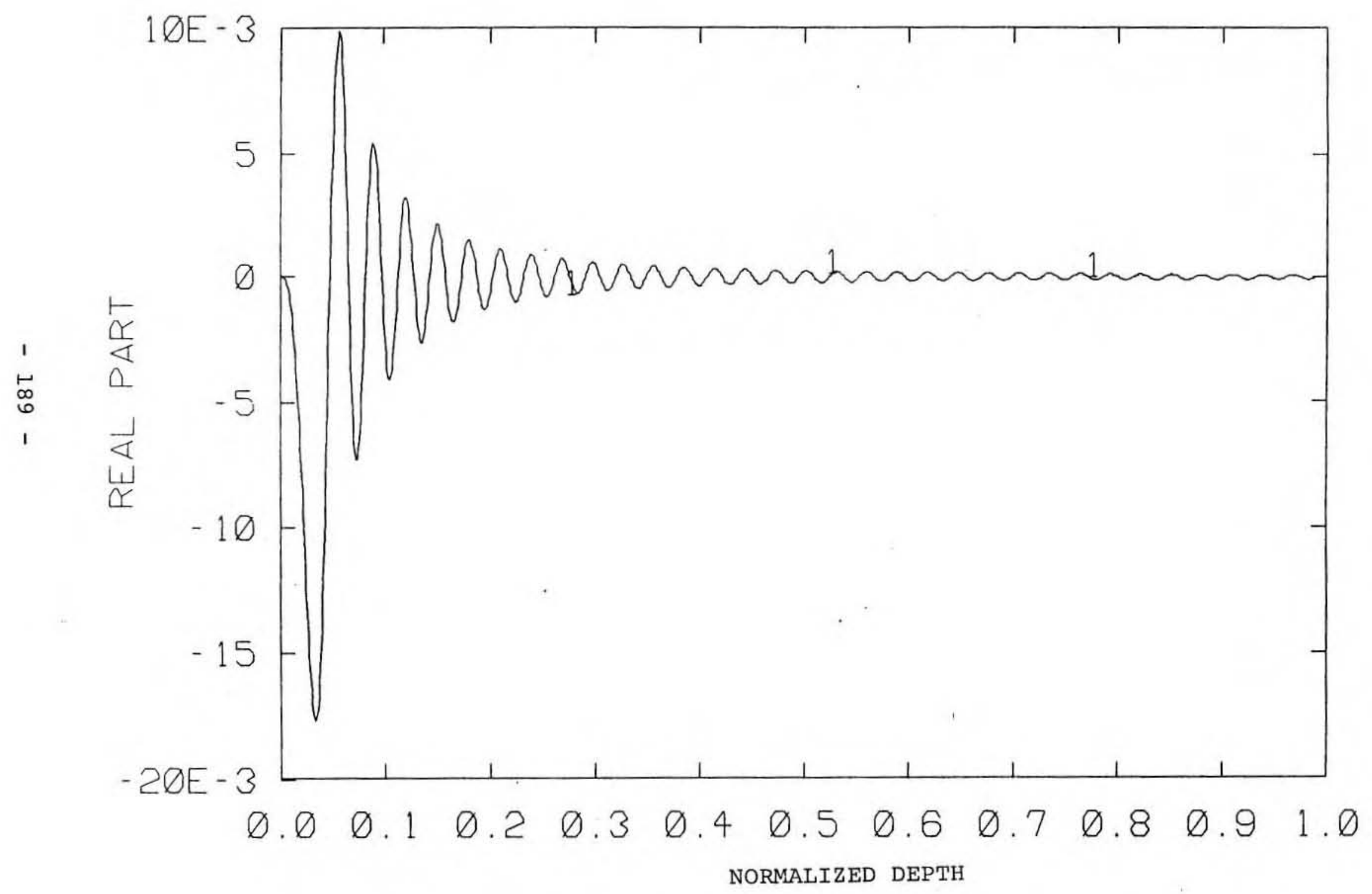

Figure 30g: Fourier Transform of Figure $30(e, f)$ 


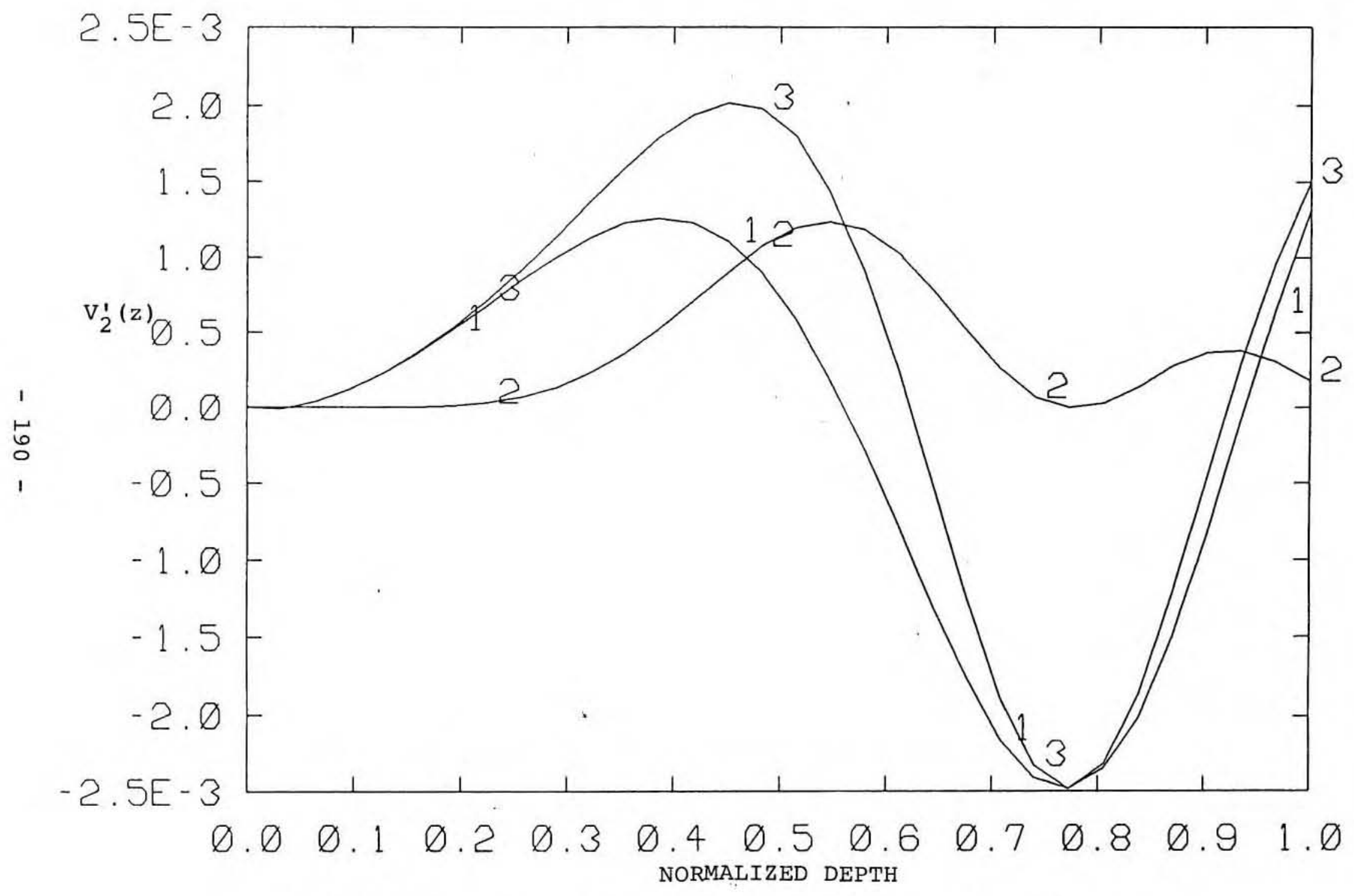
Figure 30h: Density-Dependent Potential Reconstruction $\mathrm{V}_{2}^{\prime}(\mathrm{z})$
Born:1, Correction: 2, Improved Born: 3

$\mathrm{f}=50 \mathrm{~Hz}$, Density $\rho(z)=c(z) / 1000$ 


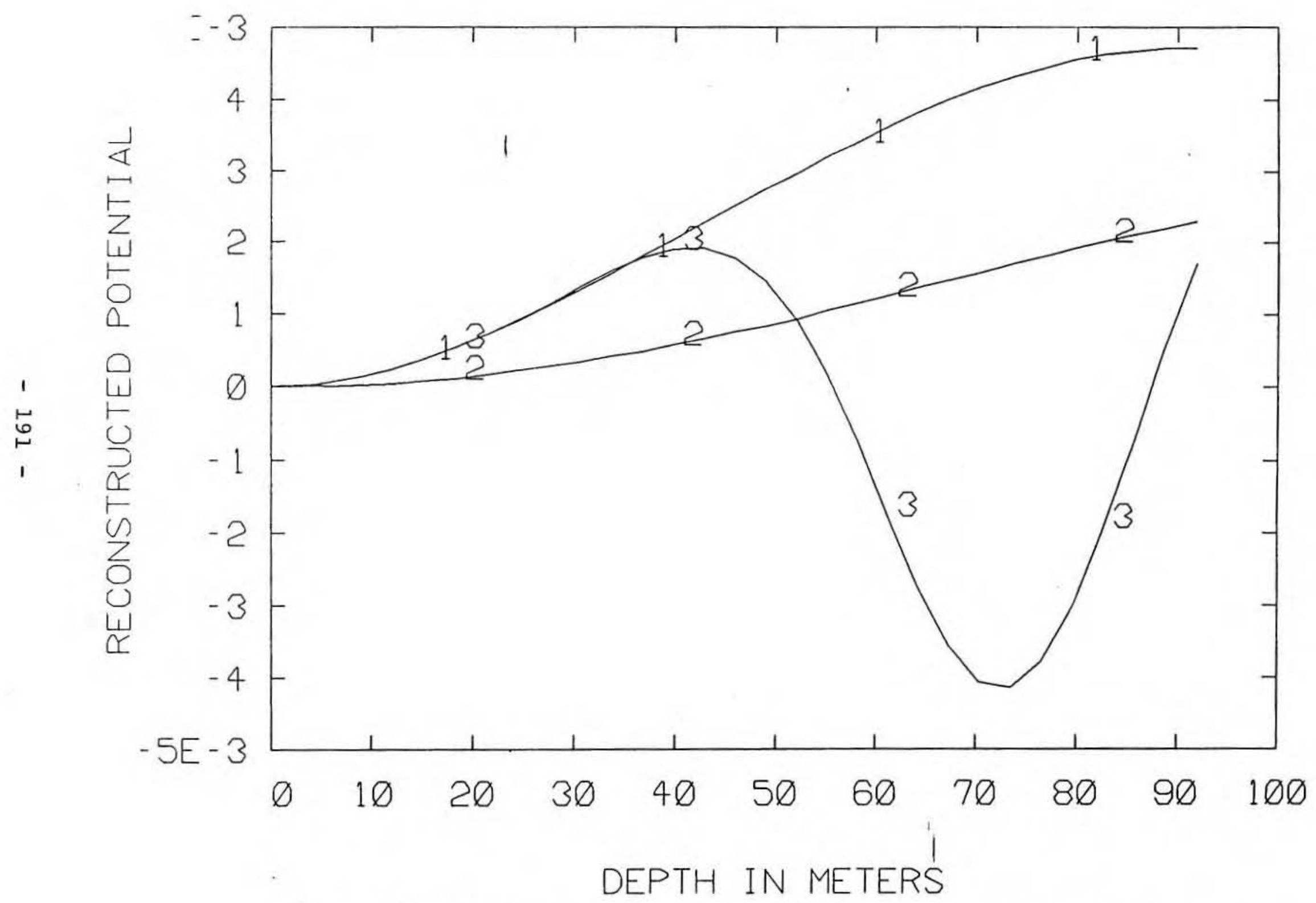

Figure 30i: Reconstructed Velocity Potential $V(z)-\rho(z)=c(z) / 1000$ 1: Original Stickler Profile, 3: Improved Born 


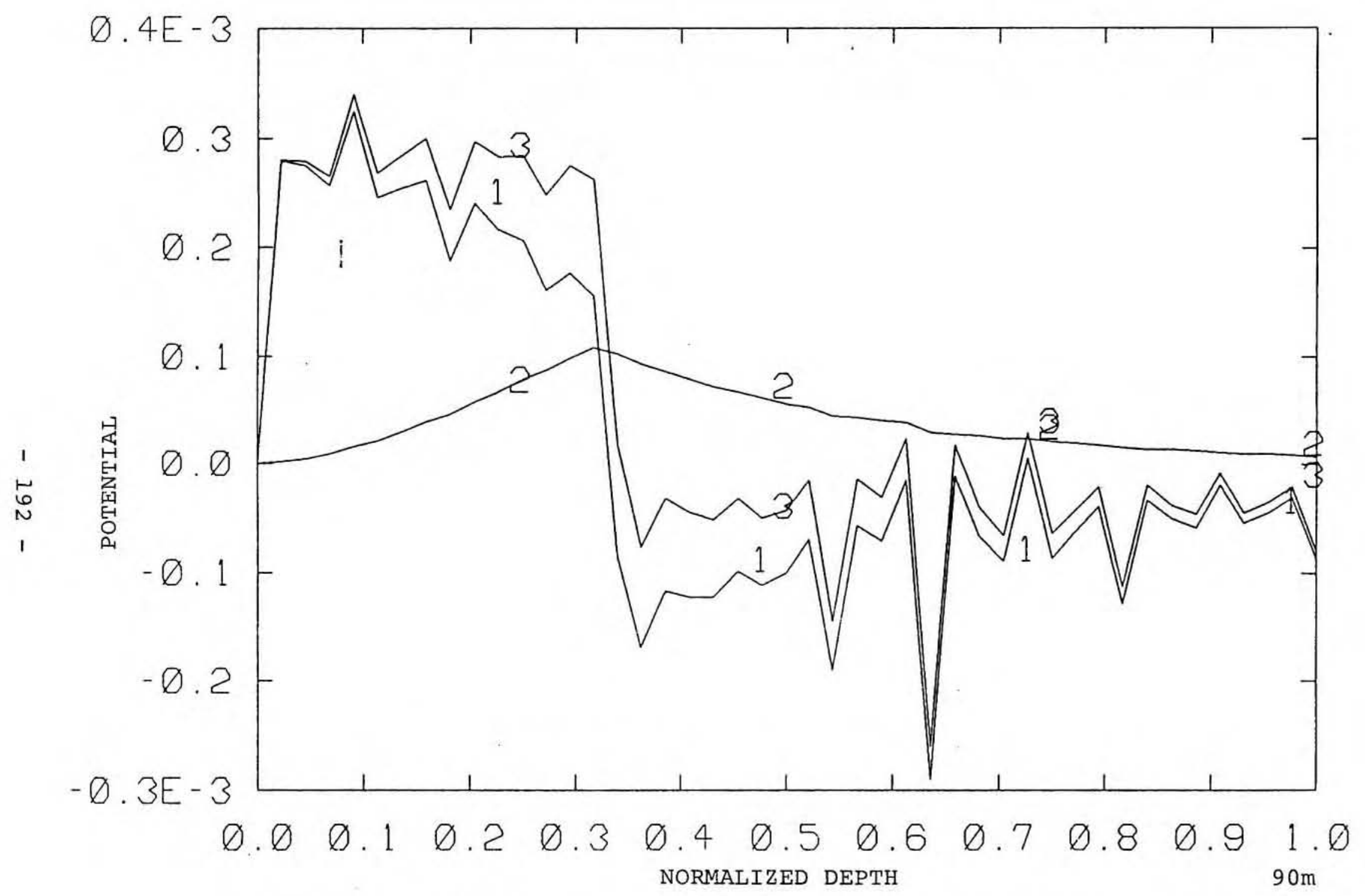

Figure 31: Improved Born Reconstruction of Window Profile $(W=45 \mathrm{~m})$ Noisy Reflection Coefficient (Zero-Mean Gaussian, $\sigma=0.1$ ) 


\section{BIBLIOGRAPHY}

1. S.R. Santaniello, F.R. diNapoli, R.K. Dullea, and P.D. Herstein, NUSC Technical Document 5337, 1976.

2. G.V. Frisk, J. Doutt, and E. Hays, "Bottom Interaction of Low-Frequency Acoustic Signals at Small Grazing Angles in the Deep Ocean", J. Acoust. Soc. Am., 69, 84-94 (1981).

3. G.V. Frisk, D.R. Mook, J.A. Doutt, E.E. Hays, M.S. Wengrovitz, and A.V. Oppenheim, "The Application to Experimental Data of a Technique for Measuring the Plane-Wave Reflection Coefficient of the Ocean Bottom", to be submitted to J. Acoust. Soc., Am.

4. G.V. Frisk, J. Doutt, and E. Hays, "Geoacoustic Models for the Icelandic Basin", J. Acoust. Soc. Am., 80, 591-600 (1986).

5. G.V. Frisk, A.V. Oppenheim, and D. Martinez, "A Technique for Measuring the Plane-Wave Reflection Coefficient of the Ocean Bottom", J. Acoust. Soc. Am., 68, 602-612 (1980).

6A. G.V. Frisk, D.R. Mook, J.A. Doutt, E.E. Hays, and A.V. Oppenheim, "The Application to Real Data of a Technique for Measuring the Plane-Wave Reflection Coefficient of the Ocean Bottom", J. Acoust. Soc. Am., 72, (S1) S97(a) (1982).

6B. D.R. Mook, G.V. Frisk, and A.V. Oppenheim, "A Hybrid Numerical/Analytic Technique for the Computation of Wave Fields in Stratified Media Based on the Hankel Transform", J. Acoust. Soc. Am., 76, 222-243 (1984).

7. D.C. Stickler, "Inverse Scattering in a stratified Medium", J. Acoust. Soc. Am., 74, 994-1005 (1983).

8. G.J. Fryer, "Reflectivity of Ocean Bottom at Low Frequency", J. Acoust. Soc. Am., 63, 35-43 (1978)

9. P.J. Vidmar and T.L. Foreman, "A Plane Wave Reflection Loss Model Including Sediment Rigidity", J. Acost. Soc. Am., 66, 1830-1835 (1979).

10. G.J. Fryer, "Compressional-Shear Wave Coupling Induced by Velocity Gradients in Marine Sediments", J. Acoust. Soc. Am., 69, 647-659 (1981). 


\section{BIBLIOGRAPHY (Continued)}

11. L.M. Brekhovskikh, Waves in Layered Media, (Academic, New York, 1960).

12. R.G. Newton, "Inversion of Reflection Data for Layered Media: a Review of Exact Methods", Geophys. J.R. Astr. Soc., 65, 191-215 (1981).

13. R. Newton, "Note on Inversion of Reflection Data for Layered Media", Geophys, J.R. Soc, 69, 571-572 (1982).

14. I.M. Gelfand and B.M. Levitan, "On the Determination of a Differential Equation from its Spectral Function", Amer. Math. Soc. Trans., Series 2, Vo1. 1, 253-304 (1955).

15. Z.S. Agranovich and V.A. Marchenko, The Inverse Problem of Scattering Theory, (Gordon and Breach, New York, 1963).

16A. L.D. Faddeyev, "The Inverse Problem in the Quantrum Theory of Scattering", translated by B. Seckler, J. Math. Phys., 44, 72-104 (1963).

16B. L.D. Faddeyev, "Properties of the S Matrix of the OneDimensional Schrodinger Equation", Am. Math. Soc. Trans., Series 2, 65, 139-166 (1964).

17. K. Chadan and P.C. Sabatier, Inverse Problems in Quantum Scattering Theory, (Springer, New York, 1977).

18. R. Burridge, "The Gelfand-Levitan, the Marchenko, and the Gopinath-Sondhi Integral Equations of Inverse Scattering Theory, Regarded in the Context of Inverse Impulse-Response Problems", Wave Motion, $\underline{2}$, 305-324 (1980)

19. I. Kay, "The Inverse Scattering Problem for Transmission Lines" in Mathematics of Profile Inversion, L. Colin, Editor, NASA TM X-62, 150, 6-2 to 6-17 (1972).

20. H.E. Moses and G.M. deRidder, Properties of Dielectrics from Reflection Coefficients in One Dimension, Lincoln Lab. Tech. Rep. 322 (1963). 
21. J.A. Ware and K. Aki, "Continuous and Discrete Inverse Scattering Problems in a Stratified Elastic Medium", J. Acoust. Soc. Am., 45, 911-921 (1969).

22. J.F. Claerbout, Fundamentals of Geophysical Data Processing, (Mc Graw-Hill, New York, 1976).

23. A.K. Jordan and S. Ahn, "Inverse Scattering Theory and Profile Reconstruction", Proc. Inst. Elec. Eng., $126,945-950$ (1979).

24. M.H. Reilly and A.K. Jordan, "The applicability of an Inverse Method for Reconstruction of Electron Density Profiles", IEEE Trans. Antennas Propagation, 29, 245-253 (1981).

25. A.K. Jordan and H.N. Kritikos, "An Application of OneDimensional Inverse Scattering Theory for Inhomogeneous Regions", IEEE Trans. Antennas Propagation, 21, 909-911 (1973).

26A. J.G. Berryman and R.R. Greene, "Discrete Inverse Methods for Elastic Waves in Layered Media", Geophysics, $\underline{45}, 213-234$ (1980)

26B. J.G. Berryman and R.R. Greene, "Discrete Inverse Scattering Theory and the Continuum Limit", Phys. Letters, 65A, 13-15 (1978)

27. A. Jacobs and R.H. Stolt, "Seismic Inversion in a Layered Medium - The Gelfand-Levitan Algorithm", Stanford Explor. Project Rep., No. 24, 109-134 (1980).

28. R. Carroll and F. Santosa, "On Complete Recovery of Geophysical Data, Math. Meth. Appl. Sci. (1982).

29. R. Carroll and F. Santosa, "Stability for the One Dimensional Inverse Problem via the Cel'fandLevitan Equation", Appl. Analysis, 13, 271-277 (1982).

30. F. Santosa, "Numerical Scheme for the Inversion of Acoustical Impedance Profile Based on the GelfandLevitan Method", Geophys. J.R. Astr. Soc., 70, 229-243 (1982). 
31. B. Gopinath and M.M. Sondhi, "Determination of the Shape of the Human Vocal Tract from Acoustical Measurements", Bell System Tech. J., 49, 1195-1214 (1970).

32. S. Coen, "Inverse Scattering of a Layered and Dispersionless Dielectric Half-Space, Part I: Reflection Data from Plane Waves at Normal Incidence", 29, 726-732 (1981).

33. S. Coen, "Density und Compressibility Profiles of a Layered Acoustic Medium from Precritical Incidence Data", Geophysics, 46, 1244-1246 (1981).

34. S. Coen, "Velocity and Density Profiles of a Layered Acoustic Medium from Common Source - Point Data", Geophysics, 47, 898-905 (1982).

35. D.H. Schaubert and R. Mittra, "A Special Domain Method for Remotely Probing Stratified Media", IEEE Trans. Antennas Propagat., 25, 261-265 (1977)

36. A. Roger, "Determination of the Index Profile of a Dielectric Plate from Scattering Data", Applied Inverse Problems, (Springer, New York, 1978).

37. P. Deift and E. Trubowitz, "Inverse Scattering on the Line", Comm. Pure Appl. Math., 23, 121-251 (1979).

38. A. Yagle and B. Levy, "The Schur Algorithm and its Applications, Tech. Report, Laboratory for Information and Decision Systems", MIT, Cambridge (1983).

39. A. Yagle and B. Levy, "Application of the Schur Algorithm to the Inverse Problem for a Layered Acoustic Medium", Tech. Report, Laboratory for Information and Decision Systems, MIT, Cambridge (1983).

40. A. Nilsen and B. Gjevik, "Inversion of Reflection Data", Geophys. Prosp., 26, 421-432 (1978).

41. H.E. Moses, "Calculation of the Scattering Potential from Reflection Coefficients", Phys. Rev., 102, 559-567 (1956). 
42. F. Santosa and W. Symes, "The Determination of a Layered Acoustic Medium Via Multiple Impedance Profile Inversions from Plane Wave Responses", Geophys. J.R. Soc., 81, 175-195 (1985).

43. F. Santosa and H. Schwetlick, "The Inversion of Acoustical Impedance Profile by Methods of Characteristics", Wave Motion, 느, 99-110 (1982).

44. D.P. Klemer and C.B. Sharpe, "An Integral Equation Associated with the Inverse Problem for Nonuniform Transmission Lines with Terminal Discontinuities", IEEE Trans. Antennas Propagation, 34, 546-553 $(1986)$.

45. J.K. Cohen and N. Bleistein, "An Inverse Method for Determining Small Variations in Propagation Speed", SIAM J. Appl. Math., 32, 784-799 (1977).

46. J.K. Cohen and N. Bleistein, "Velocity Inversion Procedure for Acoustic Waves", Geophysics, 44 , 1077-1085 (1979).

47. S.H. Gray, J.K. Cohen, and N. Bleistein, "Velocity Inversion in a Stratified Medium with Separated Source and Receiver", J. Acoust. Soc. Am., 68, 24-240 (1980).

48. S. Raz, "Direct Reconstruction of Velocity and Density Profiles from Scattered Field Data", Geophysics, 46, 832-836 (1981).

49. M. Razavy, "Determination of the Wave Velocity in an Inhomogenous Medium from the Reflection Coefficient", J. Acoust. Soc. Am., 58, 956-963 (1975).

50. M.A. Hooshayar and M. Razavy, "A Method for Constructing Wave Velocity and Density Profiles from the Angular Dependence of the Reflection Coefficient, J. Acoust. Soc. Am., 73, 19-23 (1983).

51. A.B. Weglein and S.H. Gray, "The Sensitivity of Born Inversion to the Choice of Reference Velocity: A Simple Example", Geophysics, 48, 36-38, (1983).

52. S. Gray, "The Relationship Between 'Direct, Discrete' and 'Iterative, Continuous' One-Dimensional Inverse Methods", Geophysics, 49, 54-59 (1984). 


\section{BIBLIOGRAPHY (Continued)}

53. J. Cohen and F. Hagin, "Velocity Inversion Using a Stratified Reference", Geophysics, 50, 1689-1700 (1985).

54. N. Bleistein and J. Cohen, "The Velocity Inversion Problem-Present Status, New Direction", Geophysics, 47, 1479-1511 (1982).

55. C. Rorres, J. Bai, P.C. Pedersen, and O.J. Tretiak, "Inverse Acoustic Scattering for One-Dimensional Lossy Media", J. Acoust. Soc. AM., 78, 1851-1858, $(1985)$.

56. A. Gersztenkorn, J. Bednar, and L. Lines, "Robust Iterative Inversion for the One-Dimensional Acoustic Wave Equation, Geophysics, 51, 357-368 (1986).

57. S.M. Candel, F. Defillippi, and A. Lannay, "Determination of the Inhomogenous Structure of a Medium from its Plane Wave Reflection Response, Part II: A Numerical Approximation", J. Sound Vib, 68, 583-595 $(1980)$.

58. J.A. Kong Theory of Electromagnetic Waves, (John Wiley, New York, 1975).

59. V. Bargmann, "On the Number of Bound States in a Central Field of Force", Proc. Nat. Acad. Sci. USA, 38, 961-966 (1952).

60. E.L. Hamilton, "Geoacoustic Modeling of the Sea Floor", J. Acoust. Soc. Am., 68 1313-1339 (1980).

61. S.G. Mikhlin, Integral Equations (Pergamon Press, New York, 1957).

62. H.N. Kritikos, D.L. Jaggard, and D.B. Ge, "Numerical Reconstruction of Smooth Dielectric Profiles", Proc. IEEE, 70, 295-297 (1982).

63. D.B. Ge, D.L. Jaggard, and H.N. Kritikos, "Perturbational and High Frequency Profile Inversion", IEEE Trans. Antennas Propagation, 31, 804-808 (1983). 


\section{BIBLIOGRAPHY (Continued)}

64. D.R. Mook, "The Numerical Synthesis and Inversion of Acoustic Fields Using the Hankel Transform with Application to the Estimation of the Plan Wave Reflection Coefficient of the Ocean Bottom", Sc.D. Thesis MIT/WHOI Joint Program, Cambridge, MA, and Woods Hole, MA (1983).

65. M. Abramowitz and I.A. Stegun, Handbook of Mathematical Functions, (NBS, Washington, DC, 1970).

66. A.V. Oppenheim and R.W. Schafer, Digital Signal Processing (Prentice Hall, Englewood Cliffs, 1975).

67A. S.D. Rajan and G.V. Frisk, "An Inverse Method for Obtaining the Attenuation Profile and Small Variations in the Sound Speed and Density Profiles of the Ocean Bottom", in Proceedings of the I.C.A.Congress, Symposium on Underwater Acoustics (Halifax, Nova Scotia, Canada, 16-18 July 1986).

67B. S.D. Rajan and G.V. Frisk, "An Inverse Method for Obtaining the Attenuation Profile and Small Variations in the Sound Speed and Density Profiles of the Ocean Bottom", to be submitted to J. Acoust. Soc. Am.

68. S.D. Rajan, "An Inverse Method for Obtaining the Attenuation Profile and Small Variations in the Sound Speed and Density Profiles of the Ocean Bottom", Ph.D. Thesis, MIT/WHOI Joint Program, Cambridge, MA, and Woods Hole, MA (1983).

69. M. Jaulent, "Inverse Scattering Problems in Absorbing Media", J. Math. Phys., 17, 1351-1360 (1976).

70. A. Bruckstein, B. Levy, and T. Kailath, "Differential Methods in Inverse Scatter", SIAM J. Appl. Math. (1984).

71. K.P. Bube and R. Burridge, "The One-Dimensional Inverse Problem of Reflection Seismology", SIAM Review, 25, 497-559 (1983).

72. P.C. Sabatier, "Theoretical Considerations for Inverse Scattering, Radio Science, $18,1-18$ (1983). 


\section{BIBLIOGRAPHY (Concluded)}

73. D.C. Stickler, "Application of the Trace Method for Inverse Scattering to a Medium Which Supports $\mathrm{N}$-Wave Types", unpublished report.

74. W. Symes, "Stable Solution of the Inverse Reflection Problem for a Smoothly Stratified Elastic Medium, SIAM J. Math. Anal., 12, 421-453 (1980).

75. A.B. Weglein, "The Inverse Scattering Concept and its Seismic Application", in Fitch, A.A., Ed., Developments in Geophysical Methods, 6 (Elsevier Science, 1985).

76. R.H. Stolt and A.B. Weglein, "Migration and Inversion of Seismic Data", Geophysics, 50, 2458-2472 (1985). 